\title{
Market risk of BRIC Eurobonds in the financial crisis period
}

- Dimitrios I. Vortelinos,

- Geeta Lakshmir

- $\quad$ Lincoln Business School, University of Lincoln, UK

Received 2 March 2014, Revised 23 April 2015, Accepted 23 April 2015, Available online 5 May 2015

doi:10.1016/j.iref.2015.04.012

\section{Highlights}

Most significant in terms of risk and jumps are the Chinese, among BRIC Eurobonds.

Most significant range estimator is the Yang Zhang estimator.

Higher risk and jumps for theoretical and not actual prices

Higher expiry period relates to more significant risk and jumps.

\begin{abstract}
The market risk of returns for BRIC Eurobonds has not been thoroughly analyzed via nonparametric estimation methods. The significance of risk and jumps is examined in a monthly sampling frequency. A detailed comparison upon significance of risk and jumps between BRIC Eurobonds is provided. Comparison concerns risk and jumps during the international financial crisis period: February 2007 up to February 2010. Among the BRIC countries, Chinese Eurobonds are the most significant in terms of both risk and jumps. The most significant estimator is the monthly Yang \& Zhang range across the set of BRIC Eurobonds. The shorter the expiry period, the higher is the significance of risk and jumps. This is evident in all BRIC Eurobonds. Risk and jump estimates are higher for theoretical prices rather than for actual prices according to all risk and jump significance measures.
\end{abstract}

\section{Keywords}


- BRIC Eurobonds;

- Risk;

- Jumps;

- Bond pricing;

- Financial crisis

JEL classification

- $\mathrm{G} 12$;

- $\mathrm{G} 15 ;$

- $\mathrm{G} 17$

\section{Introduction}

In the last decade, the BRIC2 countries have been researched extensively in the economics and finance literature. One of the first studies researching the significant role of the BRIC economies in the contemporary international economy's structure is Julius (2005). Recently, Aloui, Aissa, and Nguyen (2011) showed strong evidence of time-varying dependence between each of the BRIC markets and the US markets. Some of more recent studies on BRIC countries are: Cakir and Kadundi (2013) and Bekiros (2014). Fang and You (2014) investigated how explicit structural shocks that characterize the endogenous character of changes in oil prices affect three of the four BRICs' stock-market returns. Part of this BRIC literature is the BRIC Eurobonds 3 literature, which has not been extensively investigated. A recent paper studying the BRIC countries' debt markets is by Steinbock (2012). Speicifically in ths paper, the prospects for BRIC countries from the Eurozone debt crisis are studied. Peristiani and Santos (2010) reported that the extent of the dominance of the US Eurobond market globally has been reduced as the role of BRIC countries in the international Eurobond market increased. In this paper, BRIC Eurobonds are analyzed using both actual market prices and theoretical prices. Actual prices are the ones obtained in the market. Theoretical prices are obtained by a pricing model (as suggested by McCulloch, 1971) which involves fitting a smooth discount function (which is a cubic spline). Moreover, literature has also not extensively examined the market risk of BRIC Eurobonds. The present paper examines the significance of both market risk and jumps of risk series in the recent financial crisis period!4.

Market risk is measured by conditional variance (volatility) that is latent; so, market risk is not directly observable. Literature has concentrated on parametric estimators of volatility, like: (i) Generalized AutoRegressive Conditional Heteroskedasticity (GARCH), (ii) Stochastic Volatility (SV), (iii) Exponentially Weighted Moving Average (EWMA) models, among others. The ex post volatility essentially becomes observable, if the effect of the microstructure noise is low. Contemporary realized volatility estimators, as the ones employed here, minimize such 
effect. As volatility becomes observable, it can be modeled directly. Andersen and Bollerslev (1998) introduced the first and most naive realized volatility estimator, as the best nonparametric volatility estimator. Recent literature suggests that the realized volatility estimator is useful for: (i) predicting future volatility (Byun \& Kim, 2013); (ii) asset allocation in portfolios (Bandi, Russell, \& Zhu, 2008); (iii) risk management (Giot \& Laurent, 2004); and (iv) VaR computation (Clements, Galvao, \& Kim, 2008). Sevi (2015) investigated the realized volatility usefulness for modeling the convenience yield. Specifically, monthly realized volatilities and jumps explained convenience yield; whereas, jumps were detected as in Tauchen and Zhou (2010).

The present paper employs the estimation strength of many non-parametric volatility estimators; all belonging to the realized volatility literature. Estimators are classified into three groups: realized volatility estimators, range volatility estimators, and realized range-based volatility estimators. The first group of estimators studied is realized volatility estimators. The non-parametric estimator that most effectively uses data for estimation purposes is realized volatility. Andersen et al., 2001 and Andersen et al., 2003 were the first to theoretically and empirically research realized volatility estimation. There are different parameterizations for the realized volatility estimation literature. Most of the finance literature estimates realized volatility in a daily frequency via intraday data series. However, the present paper estimates monthly volatilities via daily data, because of low intraday and daily liquidity. Jiang and Tian (2005) out-of-sample compares the realized volatility to implied volatility in a monthly frequency. A recent influential study in monthly realized volatility estimation (as well as forecasting) is Busch, Christensen, and Nielsen (2011). A more recent and applied study on realized volatility estimators is Bollerslev, Osterrieder, Sizova, and Tauchen (2013). Another nonparametric volatility estimator is range. The first range estimator was suggested in Parkinson (1980). A recent study in range estimators of volatility is Louzis, XanthopoulosSisinis, and Refenes (2013). A third group of nonparametric estimators is realized rangebased volatility estimators. One of the very first papers to research this type of estimators is Martens and van Dijk (2007). A recent study in realized range-based estimators is Bannouh, Martens, and van Dijk (2013).

In the present paper, twelve nonparametric volatility estimators estimate risk. These estimators are split into three categories: realized volatility, monthly range, and realized range-based volatility. The first group includes the 5-minute unrestricted realized volatility $\left.\left(R V^{(} m^{\prime}\right)\right)$, the realized bipower variation $(B P V(m))$, a moving average-based volatility that uses

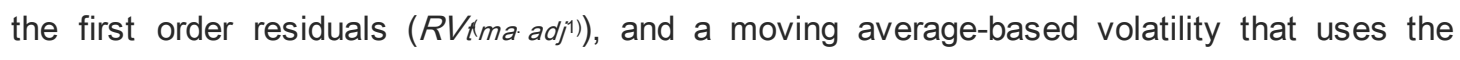
second order residuals (RVima adj $\left.j^{2}\right)$. The monthly ranges group includes the monthly Parkinson range (MRAPar), the monthly Garman \& Klass range $\left(M R^{\prime} G K\right)$, the monthly Rogers \& Satchell range $(M R(R S)$ ), and the monthly Yang \& Zhang range (MR(YZ). The third group of realized range-based volatility includes the realized Parkinson range-based volatility (RRAPar), the realized Garman \& Klass range-based volatility $(R R(G K)$, the realized Rogers \& Satchell 
range-based volatility $(R R(R S))$, and the realized Yang \& Zhang range-based volatility $(R R(Y Z)$.

Risk (volatility) series is not a continuous process; jumps make this process discontinuous. Jumps can be detected in any time interval. Literature detected and studied jumps in volatility from an intraday sampling frequency도 up to a monthly frequency‥ The present paper studies monthly jumps (in monthly volatility series). The employed detection scheme was introduced in Ait-Sahalia and Jacod (2009)

The present paper estimates volatility in a monthly frequency, because most of market participants in the Eurobonds market do not aim at intraday capital gains. They mostly trade sovereign bonds either in a daily or most probably in a monthly frequency. Most of them are fund managers or treasurers, pension or hedge fund managers or treasurers, rebalancing their portfolios monthly. So, there is no need to employ intraday high-frequency data. Moreover, there is low liquidity of Eurobonds in an intraday frequency. In this paper, we use non-parametric volatility estimators, as literature suggests due to higher robustness. Studies in the literature have recently estimated realized volatility in a monthly frequency. Afonso, Gomes, and Taamouti (2014) used, as an alternative to parametric volatility models, nonparametric measures of volatility: the absolute value and the squared returns as proxies of monthly volatilities. An, Ang, Bali, and Cakici (2014)employed the monthly realized volatility estimates as a factor in the cross-sectional relation between implied volatility shocks. Zhu and Lian (2015) provided in a monthly frequency two analytical closed-form formulae for the price of forward-start variance swap with the realized variance being defined by the actual-return realized variance and the log-return realized variance. Moreover, Lee, Paek, Ha, and Ko (2015)employed a structural VAR model for examining the relations among monthly realized volatility, market return, and aggregate equity fund flows in an international context. Seo and Kim (2015) examined the effect of investor sentiment on the relationship between the optionimplied information and the future stock return monthly realized volatility. Moreover, more accurate estimators are employed for nonparametricaly estimating monthly realized volatility in this paper.

This study contributes to the literature through the following aspects. To the best of our knowledge, this present study is the first to nonparametrically examine the significance of risk and jumps of BRIC Eurobonds. Secondly, many realized volatility estimators are employed. Risk is estimated via twelve nonparametric estimators as split into three groups (realized volatility, range, and realized range-based volatility). The significance of risk is measured via the mean magnitude of risk $(\bar{R})$ and the mean Sharpe ratio $\overline{(S R}$ ) as well. Thirdly, two jump detecton schemes are employed for risk jumps. The significance of jumps is measured via the mean magnitude of jumps $(\overline{J M})$, the mean magnitude of the jump component of risk relative to the magnitude of the continuous component $(\overline{J R})$, the average frequency of jump occurrence ( $\bar{J})$, and the average frequency of occurrence of statistically significant jumps $\left(J^{+}\right)$. Fourthly, volatility estimates concern both actual and theoretical prices. 
The remainder of the article is structured as follows. The second section describes the data and provides a descriptive analysis of returns. The third section presents the methodology. The fourth section discusses on empirical findings. The fifth section summarizes and concludes.

\section{Data}

\subsection{Data description}

The sample covers the period from February 2007 to February 2010, a total of 717 trading days or 37 months. Data relates to actual (market) prices of ten Eurobonds from all BRIC countries (Brazil, Russia, India and China). BRIC member countries together encompass over $25 \%$ of the world's land coverage, $40 \%$ of the world's population, and about $25 \%$ of the global GDP in 2010, with significant increases in global GDP share expected over the next four decades.

The sovereign bond ratings from Moody's for the BRIC countries are: Brazil (BBB -), Russia $(B B B+)$, India $(B B B-)$ and China $(A+)$. There are similarities as well as some differences between the stock exchanges of the BRIC countries. According to Table 1, China is ranked first and Russia last among BRIC countries in terms of market capitalization, market capitalization to GDP, the MSCl Emerging markets index weights and the S\&P/IFC EM Index weights. Brazil and India are ranked in between. China is also ranked first in terms of GDP growth, with India second, Russia third and Brazil last.

Table 1.

BRIC countries' stock exchanges.

\begin{tabular}{|c|c|c|c|c|c|c|}
\hline Country & $\begin{array}{l}\text { GDP } \\
\text { growth } \\
(\%)\end{array}$ & Exchange & $\begin{array}{l}\text { Market } \\
\text { capitalization }\end{array}$ & $\begin{array}{l}\text { Market } \\
\text { capitalization to } \\
\operatorname{GDP}(\%)\end{array}$ & $\begin{array}{l}\mathrm{MSCl} \text { emerging } \\
\text { markets index } \\
\text { weights }\end{array}$ & $\begin{array}{l}\text { S\&P/IFC EM } \\
\text { index weights }\end{array}$ \\
\hline Brazil & 5.08 & $\begin{array}{l}\text { BM \& } \\
\text { FBOVESPA }\end{array}$ & $1,337,248$ & 74.26 & $16.90 \%$ & $11.99 \%$ \\
\hline Russia & 5.60 & MICEX & 736,307 & 69.99 & $6.30 \%$ & $6.45 \%$ \\
\hline India & 6.07 & Bombay SE & $1,306,520$ & 90.01 & $7.50 \%$ & $7.39 \%$ \\
\hline China & 9.00 & Shanghai SE & $2,704,778$ & 100.46 & $17.90 \%$ & $17.29 \%$ \\
\hline
\end{tabular}

Notes. Table 1 reports the name of the major stock exchange of each of the BRIC countries, as well as the market capitalization, market capitalization to GDP, the country-weights in the MSCl Emerging markets index, and the S\&P/IFC EM Index weights. Market capitalization is in \$ millions. Table 1 depicts data for the year 2010 coming from the WFSE (World Federation of Stock Exchanges) historical statistics; only the GDP growth (as a \%) is provided by the World Bank.

Table 2 provides the symbol, description, country of origin, expiry year as well as an indication for either actual (market) or theoretical prices. Each country's Eurobonds market is analyzed by three Eurobonds with the only exception being India for which only one Eurobond is employed. The expiry year differs across these Eurobonds. Two Eurobonds expired in late 2010, one in 2011, one in 2012, one in 2013, three expired in 2014, one will expire in 2015 
and one in 2016. Daily bond prices have been used to estimate Eurobonds' monthly risk and monthly jumps, as the monthly frequency is appropriate not for traders but for long-term investors (mostly, pension funds) in Eurobond markets.

Table 2.

BRIC countries' Eurobonds.

\begin{tabular}{|c|c|c|c|c|}
\hline Symbol & Description & Country & Expiry year & The/Act \\
\hline$B 14_{a c t}$ & brazil_7_14_2014 & Brazil & 2014 & Act. \\
\hline$B 14_{\text {the }}$ & brazil_7_14_2014 & Brazil & 2014 & The. \\
\hline$B 11_{a c t}$ & brazil_8_7__2011 & Brazil & 2011 & Act. \\
\hline$B 11_{\text {the }}$ & brazil_8_7_2011 & Brazil & 2011 & The. \\
\hline$B 12_{a c t}$ & brazil_1_11_2012 & Brazil & 2012 & Act. \\
\hline$B 12_{\text {the }}$ & brazil_1_11_2012 & Brazil & 2012 & The. \\
\hline$R 13_{a c t}$ & russian_agri_5_16_2013 & Russia & 2013 & Act. \\
\hline$R 13_{\text {thw }}$ & russian_agri_5_16_2013 & Russia & 2013 & The. \\
\hline$R 10 a c t_{1}$ & bank_of_moscow_11_26_2010 & Russia & 2010 & Act. \\
\hline$R 10$ the $_{1}$ & bank_of_moscow_11_26_2010 & Russia & 2010 & The. \\
\hline$R 10$ act $_{2}$ & bank_of_moscow_11_29_2010 & Russia & 2010 & Act. \\
\hline$R 10$ the $_{2}$ & bank_of_moscow_11_29_2010 & Russia & 2010 & The. \\
\hline $116_{\text {act }}$ & ntpc_india_3_2_2016 & India & 2016 & Act. \\
\hline $116_{\text {the }}$ & ntpc_india_3_2_2016 & India & 2016 & The. \\
\hline C14act, & china_dev_bank_10_8_2014 & China & 2014 & Act. \\
\hline C14the, & china_dev_bank_10_8_2014 & China & 2014 & The. \\
\hline $\mathrm{C}_{14} \mathrm{act}_{2}$ & exim_china_7_29_2014 & China & 2014 & Act. \\
\hline C14 the $_{2}$ & exim_china_7_29_2014 & China & 2014 & The. \\
\hline$C 15_{a c t}$ & china_dev_bank_10_15_2015 & China & 2015 & Act. \\
\hline$C 15_{\text {the }}$ & china_dev_bank_10_15_2015 & China & 2015 & The. \\
\hline
\end{tabular}

Notes. Table 2 reports the symbol, description, country, expiry year, and the indication of actual or theoretical prices series. Theoretical prices are retrieved as in Section 3.1.

\subsection{Descriptive analysis}

Return is the logarithmic difference between two consecutive prices. Table 3 presents descriptive statistics (mean, standard deviation, skewness and kurtosis) as well as the normality hypothesis results (CVM-test and QQ-test) for returns. The mean return as well as the standard deviation are the highest for the Russian Eurobond, compared to others. Skewness and kurtosis values indicate the distributions of returns in most of the BRIC Eurobonds are skewed to the right (skewness higher than zero) and leptokurtic (kurtosis higher than three). However, the CVM and LB normality tests do not reject the null hypothesis of normality for most of the BRIC Eurobonds.

Table 3.

Returns-descriptive statistics.

$$
\text { Mean St. dev. Skew. Kurt. CVM QQ }
$$




\begin{tabular}{|c|c|c|c|c|c|c|}
\hline & Mean & St. dev. & Skew. & Kurt. & CVM & $Q Q$ \\
\hline$B 14_{a c t}$ & $-2.91 e-4$ & 0.0238 & 0.1424 & 6.05 & 0.1523 & 21.43 \\
\hline$B 14_{\text {the }}$ & $-3.24 e-4$ & 0.0203 & -0.4529 & 3.33 & 0.1341 & 36.13 \\
\hline$B 11_{a c t}$ & $-6.26 e-4$ & 0.0131 & -0.1145 & 3.22 & 0.1052 & 19.35 \\
\hline$B 11_{\text {the }}$ & $-7.32 e-4$ & 0.0204 & -0.852 & 3.58 & 0.1141 & 24.94 \\
\hline$B 12_{a c t}$ & -0.0015 & 0.0184 & -0.2023 & 5.93 & 0.2604 & 21.47 \\
\hline$B 12_{\text {the }}$ & -0.0020 & 0.0183 & -1.02 & 3.18 & 0.300 & $44.28 \unrhd$ \\
\hline$R 13_{a c t}$ & $2.65 e-4$ & 0.0642 & -1.90 & 9.37 & $0.5757 \square$ & 10.99 \\
\hline$R 13_{\text {the }}$ & $6.94 e-4$ & 0.0171 & -0.4896 & 3.20 & 0.0813 & 13.47 \\
\hline$R 10 a c t_{1}$ & $-2.94 e-4$ & 0.0576 & -1.34 & 8.72 & $0.7759 \square$ & 9.65 \\
\hline$R 10$ the $_{1}$ & -0.0019 & 0.0208 & -1.04 & 3.87 & 0.4109 & 78.83 \\
\hline$R 10 \mathrm{act}_{2}$ & $1.16 e-4$ & 0.0302 & -1.29 & 7.84 & $0.5553 \square$ & 16.41 \\
\hline$R 10$ the $_{2}$ & $-4.11 e-4$ & 0.0167 & -0.7762 & 4.05 & 0.2852 & 13.62 \\
\hline $116_{a c t}$ & 0.0014 & 0.0262 & -0.5784 & 4.82 & 0.0688 & 14.25 \\
\hline $116_{\text {the }}$ & 0.0018 & 0.0234 & 0.9926 & 5.03 & 0.0695 & 12.61 \\
\hline C14act, & 0.0018 & 0.0255 & -1.56 & 9.04 & 0.2282 & 22.71 \\
\hline C14 the ${ }_{1}$ & 0.0023 & 0.0211 & -0.1093 & 3.71 & 0.0541 & 21.56 \\
\hline$C 14$ act $_{2}$ & 0.0022 & 0.0461 & 0.2685 & 12.19 & $0.5929 \unrhd$ & 18.03 \\
\hline C14 the $_{2}$ & 0.0020 & 0.0189 & -0.0681 & 3.58 & 0.0933 & 23.19 \\
\hline$C 15_{a c t}$ & 0.0029 & 0.1711 & 0.0935 & 15.14 & $1.56 \square$ & 14.86 \\
\hline$C 15_{t h w}$ & 0.0022 & 0.0256 & 0.1171 & 4.07 & 0.0585 & 29.32 \\
\hline
\end{tabular}

Notes. The mean, standard deviation, skewness and kurtosis values as well as the CVM and QQ test statistics are reported. All descriptive statistics are reported for either actual returns (indicated by act) or theoretical returns (indicated by the).

Indicates significance in $5 \%$ significance level.

\section{Empirical methodology}

Bond prices employed, used both actual market prices and theoretical prices. Returns are produced for both actual and theoretical prices. Monthly point estimates of volatility are estimated through three groups of estimators: realized volatility, range and realized rangebased volatility. Then, monthly jumps are detected from two different detection schemes.

\subsection{Bond pricing}

Using bond prices is more reliable than using yields. This is because yields are retrieved from actual bond prices and may be depended on different maturities and coupons. The pricing model involves fiting a smooth discount function to information obtained from observed prices of straight bonds with various coupons and maturities by estimating the coefficients for a linear combination of smooth approximating functions forming a cubic spline. Any coupon bond price maturing at par value and paying a coupon at timeican be expressed as: 
equation(1)

$P+A I=C \cdot \sum_{i=1}^{T} \frac{1}{\left(1+R_{i}\right)^{i}}+\frac{100}{\left(1+R_{n}\right)^{n}}$

where $P=$ clean price or the price quoted in the market (as $\%$ of par

value), $C=$ coupon, $R_{i}=$ discount rate applicable for period $i$ with $T$ as the final maturity date.

Replacing $\frac{1}{\left(1+R_{i}\right)^{i}}$ by, returns

equation(2)

$P+A I=C \cdot \sum_{i=1}^{T} d_{i}+100 \cdot d_{n}$

The discount function $d_{i}$ can be expressed as a combination of smooth approximating functions and defines the present value of 1 unit of any numeraine receivable in $i$ years. McCulloch, 1971 and McCulloch, 1975 suggested that the discount function $d_{i}$ can be expressed as:

equation(3)

$d(i)=1+\sum_{f=1}^{k} a_{i} f_{i}(i)$

where $k f(l)$ functions are chosen (the value of $k$ varying with the exact model) to estimate $d(\lambda)$ by a cubic spline and the $a_{j}$ are the estimated parameters of the linear regression. The $f_{i}(I)$, $(j=1, \ldots, k)$ are chosen so that $f_{j}(0)=0$ to force $d(0)=1$ and to enable it to be smooth and monotonically nonincreasing. Substituting $d_{i}$ with $d(\lambda)$ in the $P+A$ /equation, the price of a bond maturing in $T$ months and paying a coupon at time $i$ can be expressed as follows:

equation(4)

$P+A I=C \cdot \sum_{i=1}^{T}\left[1+\sum_{j=1}^{k} a_{j} f_{j}(n)\right]+100 \cdot\left[1+\sum_{j=1}^{k} a_{j} f_{j}(n)\right]$.

In case of a discrete time, it is employed a discount function with two cubic splines, $k=5$ and $\sum f=1 k a i f(\lambda)=a i+\beta k+\gamma^{\beta}+\gamma_{1} D V_{1} i\left(i-t_{1} \square\right)^{3}+\gamma_{2} D V_{2} i\left(i-t_{2} \square\right)^{3}$. Then the discount factor is equation(5)

$D(i)=1+a i+\beta i^{2}+\gamma i^{3}+\gamma_{1} D V_{1} i\left(i-t_{1}^{*}\right)^{3}+\gamma_{2} D V_{2} i\left(i-t_{2}^{*}\right)^{3}$

where $D V_{1}$ and $D V_{2}$ are dummy variables shifting the cubic term of the polynomial for time points. These are the knot points for the cubic spline. When $D(l)$ is substituted in the $P+A$ / equation and an error term is added then, the final form of the pricing model is: equation(6)

$$
P+A I=\begin{gathered}
C \cdot \sum_{i=1}^{T}\left[T+a \sum h_{i}+\beta \sum h_{i}^{2}+\gamma \sum h_{i}^{3}+\gamma_{1} \sum D V_{1} h_{i}\left(h_{i}-t_{1}^{*}\right)+\gamma_{2} \sum D V_{2} h_{i}\left(h_{i}-t_{2}^{*}\right)^{3}\right]+ \\
100 \cdot\left[1+a h_{T}+\beta h_{T}^{2}+\gamma h_{T}^{3}+\gamma_{1} D V_{1} h_{T}\left(h_{T}-t_{1}^{*}\right)^{3}+\gamma_{2} D V_{2} h_{T}\left(h_{T}-t_{2}^{*}\right)^{3}\right]+e
\end{gathered}
$$


where $P$ is the clean price, $A /$ is the accrued coupon, $T$ is the total number of coupons left, $h$ is the date to the first coupon, $i=1$ is the number of coupons left to maturity (up to $T$ ) and $h_{i}$ is the date of the last cash flow. $D V$ represents dummy variables representing the spline knots if time left to maturity of the bond is greater than $t_{(-)}{ }^{*}$. Taking a large cross section of bonds in a market at a point in time with differing market prices, of diverse coupons and times to maturities and using regression allows the estimation of $a, \beta, \gamma_{1} \gamma_{1}$, and $\gamma_{2}$ using the last equation. The error term in the regression ensures that random effects are captured. Repeating this exercise over time ensures a time series of $a, \beta, \gamma, \gamma_{1}$, and $\gamma_{2}$.

The estimates of bond prices via the above bond pricing method return the so-called theoretical bond prices, which are indicated as 'the', and market prices are indicated as 'act. The risk and jumps of BRIC Eurobonds are compared across ten Eurobonds and the four countries as well as across twelve volatility estimators which are split in three groups (realized volatility, range, and realized range-based volatility). Each group consists of four estimators.

\subsection{Realized volatility estimators}

All realized volatility estimators provide monthly point estimates by using daily returns. Andersen et al. (2001) suggested the unrestricted realized volatility estimator $(R V A m)$ : equation(7)

$R V_{t}^{(m)}=\sum_{i=1}^{m} r_{i n}^{2}$

where $t$ is the indication of the month, $i$ indicates the trading day in a specific $t$ month and $m$ is the number of trading days per month across all realized volatility and range estimators. This notation is consistent across all volatility estimators. Barndorff-Nielsen, Hansen, Lunde, and Shephard (2011)theoretically and empirically examined the realized bipower variation $(B P V(m))$. In literature, this estimator is employed to detect jumps because the realized bipower variation has no jumps.

equation(8)

$B P V_{t}^{(m)}=\mu_{p}^{-2} \sum_{i=2}^{m}\left|r_{i, m}\right|\left|r_{i-1, m}\right|$

where $\mu p=\mathrm{E}(|Z| p)$ is the mean of the $p$ th absolute moment of a standard normal distribution. Hansen, Large, and Lunde (2008) constructed a moving average-based volatility estimator that uses the first order MA(1) residuals ( $R V t$ ma $\left.a j^{1}\right)$ ):

equation(9)

$R V_{t}^{\langle m a . a d j 1\}}=(1-\hat{\theta}) \sum_{i=1}^{\mathrm{m}}\left(\hat{e}_{i, \mathrm{~m}}\right)^{2}$ 
where $r_{i_{1} \mathrm{~m}}=e_{i, \mathrm{~m}}-\hat{\vartheta}_{\mathrm{t}} \mathrm{e}_{\mathrm{i}-1, \mathrm{~m}}$, and $\hat{\vartheta}_{\mathrm{t}}$ is estimated for each the month. The aim is to reduce the autocorrelation in daily returns. This was evident in the QQ-test statistic values in Table 3 as explained in Section 2.2. Hansen et al. (2008) with Bandi, Russell, and Yang (2008) also proposed a moving average-based volatility estimator that uses the q order MA(q) residuals. The $\mathrm{q}$ order is selected according to the AIC criterion. This estimator should be more accurate in case of more than one MA orders (RVtma adj)): equation(10)

$R V_{t}^{(m a . a d j 2)}=\frac{\left(1-\hat{\vartheta}_{1}-\ldots-\hat{\vartheta}_{\mathrm{q}}\right)^{2}}{1+\hat{\vartheta}_{1}^{2}+\ldots+\hat{\vartheta}_{\mathrm{q}}^{2}} R V_{t}^{(m)}$

where $r_{i, \mathrm{~m}}=e_{i, m}-\hat{\vartheta}_{1} \mathrm{e}_{\mathrm{i}-1, \mathrm{~m}}-\ldots-\hat{\vartheta}_{\mathrm{q}} \mathrm{e}_{\mathrm{i}-\mathrm{q}, \mathrm{m}}$ and $\left.R V_{k m}\right)$ is the unrestricted realized volatility estimator.

\subsection{Range-based estimators}

Range is the difference between the highest and lowest price. Range estimators are split into two categories: monthly ranges, and realized range-based volatility estimators. The monthly range estimators use the highest and lowest monthly prices per month and symbolized as $M R_{t}$. The range estimators, also estimated monthly, using the highest and lowest daily prices per day are entitled as realized range-based volatility estimators and symbolized as $R R_{t .}$. The present paper examines four monthly ranges as well as their corresponding four realized range-based estimators. These estimators are: Parkinson, Garman \& Klasss, Rogers \& Satchell, and Yang \& Zhang; either monthly or realized.

\subsubsection{Monthly ranges}

Parkinson (1980) defined and empirically analyzed the range estimator. That is why the first version of a range estimator is entitled as Parkinson range. As far as the sampling frequency of the estimator is monthly, it can be called monthly Parkinson estimator: equation(11)

$M R_{t}^{(\text {Por })}=\frac{1}{4 \ln 2} \sum\left[\ln \left(P_{h, l}(m) / P_{l, l}(m)\right)\right]^{2}$

where $P_{h, t}(m)$ is the highest monthly price (the highest price of the month) and $P_{l, t}(m)$ is the lowest monthly price (the lowest price of the month). Garman and Klass (1980) extended the Parkinson estimator to:

equation(12)

$M R_{t}^{(G K)}=\frac{1}{n} \sum\left\{\begin{array}{c}0.511 \cdot\left[\ln \left(P_{h, t}(m) / P_{l, t}(m)\right)\right]^{2}-0.019 \cdot \ln \left(P_{c, t}(m) / P_{o, \ell}(m)\right) \cdot \ln \left(P_{h, \ell}(m) \cdot P_{l, t}(m) / P_{o, t}^{2}(m)\right) \\ -2 \cdot \ln \left(P_{h, l}(m) / P_{o, l}(m)\right) \cdot \ln \left(P_{l, \ell}(m) / P_{o, \ell}(m)\right)-0.383 \cdot\left[\ln \left(P_{c, \ell}(m) / P_{o, t}(m)\right)\right]^{2}\end{array}\right\}$

where $n$ is the total number of monthly observations, $P_{c, t}(m)$ is the monthly close price (the closing price per month) and $P_{o, t}(m)$ is the monthly open price (the opening price per 
month). Rogers and Satchell (1991) extended the Parkinson estimator, in a similar way to Garman and Klass (1980) estimator, via incorporating monthly open and close prices apart from the monthly high and low prices:

equation(13)

$M R_{\ell}^{(R S)}=\frac{1}{n} \sum\left\{\ln \left(P_{h, l}(m) / P_{c, \ell}(m)\right) \cdot \ln \left(P_{h, l}(m) / P_{o, \ell}(m)\right)+\ln \left(P_{l,}(m) / P_{c \ell}(m)\right) \cdot \ln \left(P_{l, \ell}(m) / P_{o \ell,}(m)\right)\right\}$

Yang and Zhang (2000) incorporated a term for the closed market variance (that is the overmonth variance; i.e. a month-effect). So, the monthly Yang and Zhang estimator is defined as: equation(14)

$M R_{t}^{(Y Z)}=\frac{1}{n-1} \sum\left[\ln \left(P_{o, \ell}(m) / P_{c, t}(m)-P_{\overline{0}, \ell}(m)\right)\right]^{2}+\frac{k}{n-1} \sum\left[\ln \left(P_{c, t}(m) / P_{o, t}(m)-P_{z, \ell}(m)\right)\right]^{2}+(1-k) \cdot M R_{t}^{(R S)}$

where $n$ is the number of months, $P_{\mathcal{Z},}(m)=\frac{1}{n} \sum\left[\ln \left(P_{c, \ell}(T) / P_{0, \ell}(T)\right)\right]$

$$
\left.P_{z, \ell}(T)=\frac{1}{n} \sum\left[\ln \left(P_{o, \ell}(T) / P_{c, t-1}(T)\right)\right]_{\text {and }} \quad k=\frac{0.34}{1.34+\frac{n+1}{n-1}} . M R t R S\right) \text { is the monthly Rogers \& }
$$

Satchell range estimator, $P_{c, t}(T)$ is the monthly close price, $P_{o, t}(T)$ is the monthly open price, $P_{h, t}(T)$ is the monthly high price, $P_{l, t}(T)$ is the monthly low price, $P_{\bar{c}, t}(T)$ is the average monthly close price (average value of all monthly close prices) and $P_{\bar{o}, t}(T)$ is the average monthly open price (average value of all monthly open prices).

\subsubsection{Realized range-based}

When the four range-based estimators are estimated monthly via daily data, they are known as realized range-based estimators. The realized Parkinson range-based volatility estimator is suggested in Martens and van Dijk (2007) (RRtPar) as:

equation(15)

$R R_{t}^{(\text {Par })}=\frac{1}{4 \log (2)} \sum_{i=1}^{m}\left(h_{i, m}-t_{l, m}\right)^{2}$

where $m$ is the number of trading days per month, $h i, m=\ln (P h(i, m))$, and $l i, m=\ln (P /(i, m))$ are the within the $i$-th daily interval (per day; daily) high and low logarithmic prices. The realized Garman and Klass range-based estimator $\left(R R^{\wedge}\left(G^{\prime}\right)\right.$ is:

equation(16)

$R R_{t}^{(G K)}=\frac{1}{n} \sum_{i=1}^{m}\left[0.511 \cdot R_{i, \mathrm{~m}, 1}-0.019 \cdot R_{i, \mathrm{~m}, 2} \cdot R_{i, \mathrm{~m}, 3}-2 \cdot R_{i, \mathrm{~m}, 4} \cdot R_{i, \mathrm{~m}, 5}-0.383 \cdot R_{i, \mathrm{~m}, 2}^{2}\right]$

where $n$ is the number of

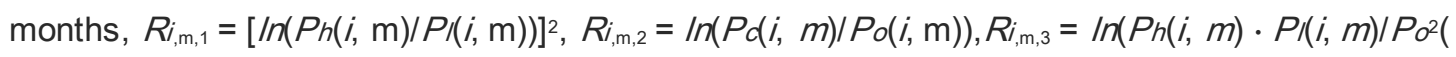
$i, \mathrm{~m})), R_{i, m_{, 4}}=\ln \left(P_{h}(i, \mathrm{~m}) / P_{o}(i, \mathrm{~m})\right)$, and $R_{i, \mathrm{~m}, 5}=\ln \left(P /(i, \mathrm{~m}) / P_{o}(i, \mathrm{~m})\right)$. Rogers and Satchell (1991)'s 
estimator can also be estimated as a realized range-based volatility estimator. So, the realized Rogers and Satchell range-based estimator (RRtRS) is given by:

equation(17)

$R R_{t}^{(R S)}=\frac{1}{n} \sum_{i=1}^{m}\left[R_{i, \mathrm{~m}, 1}^{\prime} \cdot R_{i, \mathrm{~m}, 2}+\dot{R}_{i, \mathrm{~m}, 3} \cdot \dot{R}_{i, \mathrm{~m}, 4}^{\prime}\right]$

where $\dot{R}_{i, \mathrm{~m}, 1}=\ln \left(P_{h}(i, m) / P_{c}(i, m)\right),{ }^{R_{i, \mathrm{~m}, 2}}=\ln \left(P_{h}(i, m) / P_{o}(i, \mathrm{~m})\right),{ }^{\prime}{ }_{i, \mathrm{~m}, 3}=\ln \left(P^{\prime}(i, m) / P_{c}(i, m)\right)$, and $\dot{R}_{i, \mathrm{~m}, 4}=\ln \left(P_{/}(i, m) / P_{o}(i, m)\right)$. Finally, the realized Yang and Zhang range-based volatility estimator $(R R A Y Z)$ is given by the following equation:

equation(18)

$R R_{t}^{(Y Z)}=\frac{1}{n-1} \sum_{i=1}^{m}\left(R_{i, \mathrm{~m}, 1}^{*}\right)^{2}+\frac{k}{n-1} \sum_{i=1}^{m}\left(R_{i, \mathrm{~m}, 2}^{*}\right)^{2}+(1-k) \cdot R R_{t}^{(R S)}$

where $R_{i, m, 1} \square=\ln \left(P_{o}(i, \mathrm{~m}) / P_{c}(i, m)-P_{\bar{o}}(i, m)\right), R_{i, m, 2}^{*}=\ln \left(P_{c}(i, m) / P_{o}(i, m)-P_{\bar{c}}(i, m)\right)$

$P_{\bar{o}}(i, m)=\frac{1}{n} \sum\left[\ln \left(P_{0}(i, m) / P_{c}(i-1, m)\right)\right], P_{\bar{\tau}}(i, m)=\frac{1}{n} \sum\left[\ln \left(P_{c}(i, m) / P_{o}(i, m)\right)\right]$ and $k=\frac{0.34}{1.34+\frac{n+1}{n-1}}$

\subsection{Jumps}

The detection scheme employed to detect jumps on the monthly volatility series was introduced in Ait-Sahalia and Jacod (2009) and further examined in Ait-Sahalia and Jacod (2011) and Ait-Sahalia, Jacod, and Li (2012).

All three papers detect a jump in volatility series when there is a significant difference between the realized quarticity of a specific sampling frequency and a multiple of it. The critical value for the test of this jump detection scheme is

equation(19)

$\mathrm{Fa}=2-\Phi \mathrm{a} \cdot(\mathrm{V}) 1 / 2 \mathrm{I}\left(|\mathrm{Vt}|>\mathrm{c}_{1}\right) \mathrm{Vt}+\mathrm{I}\left(|\mathrm{Vt}|<\mathrm{c}_{2}\right) \mathrm{Vt}$

where $c_{1}=0.95, c_{2}=0.05$, and $V_{t}$ is any of either realized volatility estimators or range estimators explained in the previous subsection,

$$
V=\frac{160 \cdot(m) \cdot\left(\frac{(m)^{3}}{\pi^{-1 / 2} \cdot 16 \cdot \gamma(9 / 2)} \cdot \sum_{i=1}^{m}\left|r_{i m, 1}\right|^{8}\left|r_{i, m, 2}\right|\right)}{3 \cdot\left(\left.\frac{\langle m\rangle}{\left.\pi^{-1,2} \cdot 4 \cdot \gamma / 2 / 2\right)} \cdot \sum_{i=1}^{m}\left|r_{i m, 1}\right|\right|^{4}\left|r_{i, m, 2}\right|\right)^{2}},
$$

$r_{i, m, 1}=\ln \left(P_{c_{1} 1}(i, m) / P_{c_{1}, 1}(i-1, m)\right), r_{i, m_{, 2}}=\ln \left(P_{c_{2}, 2}(i, m) / P_{c_{2}}(i-1, m)\right), P_{c_{1}}(i, m)$ is the daily close prices, and $P_{c_{2}}(i, m)$ is the daily close prices for the multiple of the first sampling (i.e. daily) frequency (in $P_{c, 1}(i, m)$ ). The standardized test statistic is $S_{\text {test }}=\frac{S-2}{V^{1 / 2}}$ 
where $\left.\left.\quad \sum_{i=1}^{\sum_{i=1}^{m}\left|r_{i, m, 1}\right|^{4}}\right|_{i, m, 2}\right|^{4}$. There are jumps for a month, when $S<F_{a}$. The empirical results reported below are relied on a significance level of $a=5 \%$.

equation(20)

$\mathrm{JMt}=\max (|\mathrm{Vt}-(\mathrm{V}) 1 / 2|, 0)$

The jump part $\left(J M_{t}\right)$ of any $V_{t}$ estimator is estimated as in Andersen, Bollerslev, Frederiksen, and Nielsen (2010) 9 . Jump frequency $\left(J F_{t}\right)$ is the frequency of occurrence of monthly jump upon the total number of months in sample; so, it is the number of months that jumps are detected, is expressed as a percentage to the total number of months for the examined (either before or after) time period. The indicator of the existence of at least one jump per month can be depicted as: $B t=(J M t \neq 0)$.

\section{Empirical findings}

All measures are based on average values of monthly point estimates of risk and jumps. Risk is measured via the mean magnitude of risk $(\bar{R})$ and the mean Sharpe ratio $(\overline{S R})$ as well. Results for risk are reported inTable 6 and Table 7 accordingly. The significance of jumps is measured via the mean magnitude of jumps $(\overline{J M})$, the mean ratio of the magnitude of the jump component of risk relative to the magnitude of the continuous component $(\overline{J R})$, and the average frequency of jump occurrence $(\bar{J})$. Results for jumps are reported in Table 8, Table 9 and Table 10 respectively.

Table 4.

Risk-descriptive statistics-skewness and kurtosis.

\begin{tabular}{|c|c|c|c|c|c|c|c|c|c|c|c|c|}
\hline & $\begin{array}{l}R V_{t} \\
m\end{array}$ & $\begin{array}{l}B P V_{t} \\
(m)\end{array}$ & $\begin{array}{l}R V_{t m a} \\
\left.a d^{\prime \prime}\right)\end{array}$ & $\begin{array}{l}R V_{t m a} \\
\left.a d^{2}\right)\end{array}$ & $\begin{array}{l}\text { MRt } \\
\text { Par }\end{array}$ & ${ }_{N}^{M R t G}$ & $\begin{array}{l}M R_{t R} \\
\mathcal{S}\end{array}$ & $\begin{array}{l}M R t Y \\
Z\end{array}$ & $\begin{array}{l}R R_{t} \\
\text { Par }\end{array}$ & $\begin{array}{l}R R_{t} \\
G K\end{array}$ & $\begin{array}{l}R R t R \\
S\end{array}$ & $\begin{array}{l}R R t \\
Y Z\end{array}$ \\
\hline$B 14_{a c}$ & $\begin{array}{l}5.54 \\
(32.4 \\
4)\end{array}$ & $\begin{array}{l}5.59 \\
(32.8 \\
5)\end{array}$ & $\begin{array}{l}5.60 \\
(32.86)\end{array}$ & $\begin{array}{l}5.56 \\
(32.56)\end{array}$ & $\begin{array}{l}5.38 \\
(31.0 \\
7)\end{array}$ & $\begin{array}{l}-2.12 \\
(8.41)\end{array}$ & $\begin{array}{l}-2.11 \\
(7.63)\end{array}$ & $\begin{array}{l}-2.06 \\
(7.36)\end{array}$ & $\begin{array}{l}5.57 \\
(32 . \\
7)\end{array}$ & $\begin{array}{l}5.57 \\
(32 . \\
69)\end{array}$ & $\begin{array}{l}5.60 \\
(32.9 \\
1)\end{array}$ & $\begin{array}{l}5.54 \\
(32 . \\
47)\end{array}$ \\
\hline $\begin{array}{l}B 14_{\text {th }} \\
e\end{array}$ & $\begin{array}{l}2.75 \\
(9.69 \\
)\end{array}$ & $\begin{array}{l}3.18 \\
(12.0 \\
8)\end{array}$ & $\begin{array}{l}3.05 \\
(11.34)\end{array}$ & $\begin{array}{l}4.12 \\
(20.42)\end{array}$ & $\begin{array}{l}2.49 \\
(8.16 \\
)\end{array}$ & $\begin{array}{l}0.208 \\
7 \\
(1.99)\end{array}$ & $\begin{array}{l}0.971 \\
0 \\
(3.54)\end{array}$ & $\begin{array}{l}0.985 \\
3 \\
(3.57)\end{array}$ & $\begin{array}{l}2.74 \\
(9.5 \\
9)\end{array}$ & $\begin{array}{l}2.75 \\
(9.6 \\
1)\end{array}$ & $\begin{array}{l}3.62 \\
(16.3 \\
7)\end{array}$ & $\begin{array}{l}3.02 \\
(11 . \\
46)\end{array}$ \\
\hline$B 11_{a c}$ & $\begin{array}{l}29.4 \\
6 \\
(30.2 \\
1)\end{array}$ & $\begin{array}{l}5.53 \\
(30.6 \\
2)\end{array}$ & $\begin{array}{l}6.51 \\
(30.69)\end{array}$ & $\begin{array}{l}6.43 \\
(30.67)\end{array}$ & $\begin{array}{l}5.16 \\
(29.4 \\
6)\end{array}$ & $\begin{array}{l}-1.14 \\
(5.53)\end{array}$ & $\begin{array}{l}-1.62 \\
(6.51)\end{array}$ & $\begin{array}{l}-1.60 \\
(6.43)\end{array}$ & $\begin{array}{l}5.27 \\
(30 . \\
43)\end{array}$ & $\begin{array}{l}1.06 \\
(5.3 \\
6)\end{array}$ & $\begin{array}{l}3.30 \\
(20.4 \\
8)\end{array}$ & $\begin{array}{l}3.40 \\
(20 . \\
56)\end{array}$ \\
\hline $\begin{array}{l}B 11_{\text {th }} \\
e\end{array}$ & $\begin{array}{l}1.36 \\
(4.04 \\
)\end{array}$ & $\begin{array}{l}1.86 \\
(6.53 \\
)\end{array}$ & $\begin{array}{l}2.04 \\
(7.58)\end{array}$ & $\begin{array}{l}2.29 \\
(8.48)\end{array}$ & $\begin{array}{l}1.11 \\
(2.75 \\
)\end{array}$ & $\begin{array}{l}0.300 \\
8 \\
(2.49)\end{array}$ & $\begin{array}{l}0.472 \\
(3.02)\end{array}$ & $\begin{array}{l}0.475 \\
6 \\
(3.03)\end{array}$ & $\begin{array}{l}2.57 \\
(9.8 \\
8)\end{array}$ & $\begin{array}{l}1.25 \\
(4.0 \\
0)\end{array}$ & $\begin{array}{l}0.336 \\
2 \\
(5.75)\end{array}$ & $\begin{array}{l}1.34 \\
(6.8 \\
6)\end{array}$ \\
\hline$B 12_{a c}$ & $\begin{array}{l}5.28 \\
(30.4\end{array}$ & $\begin{array}{l}5.37 \\
(31.1\end{array}$ & $\begin{array}{l}5.36 \\
(31.11)\end{array}$ & $\begin{array}{l}5.46 \\
(31.79)\end{array}$ & $\begin{array}{l}5.25 \\
(30.0\end{array}$ & $\begin{array}{l}-1.45 \\
(6.50)\end{array}$ & $\begin{array}{l}-1.38 \\
(5.09)\end{array}$ & $\begin{array}{l}-1.35 \\
(4.98)\end{array}$ & $\begin{array}{l}5.38 \\
(31 .\end{array}$ & $\begin{array}{l}5.37 \\
(31 .\end{array}$ & $\begin{array}{l}5.53 \\
(32.4\end{array}$ & $\begin{array}{l}5.30 \\
(30 .\end{array}$ \\
\hline
\end{tabular}




\begin{tabular}{|c|c|c|c|c|c|c|c|c|c|c|c|c|}
\hline & $\begin{array}{l}R V_{t} \\
m\end{array}$ & $\begin{array}{l}B P V t \\
(m)\end{array}$ & $\begin{array}{l}R V_{t m a} \\
\left.a f^{\prime \prime}\right)\end{array}$ & $\begin{array}{l}R V_{t m a} \\
a d^{(2)}\end{array}$ & $\begin{array}{l}M R_{t} \\
P_{a r}\end{array}$ & $\begin{array}{l}M R_{t G} \\
K\end{array}$ & $\begin{array}{l}M R_{t R} \\
\mathcal{S}\end{array}$ & $\begin{array}{l}M R_{t} Y \\
Z\end{array}$ & $\begin{array}{l}R R_{t} \\
\text { Part }\end{array}$ & $\begin{array}{l}R R_{t} \\
G K\end{array}$ & $\begin{array}{l}R R_{t} R \\
\mathcal{S}\end{array}$ & $\begin{array}{l}R R_{t} \\
Y Z\end{array}$ \\
\hline & 6) & 2) & & & 9) & & & & 20) & 17) & 2) & 61) \\
\hline $\begin{array}{l}B 12_{\text {th }} \\
e\end{array}$ & $\begin{array}{l}5.12 \\
(29.1 \\
2)\end{array}$ & $\begin{array}{l}5.51 \\
(32.2 \\
3)\end{array}$ & $\begin{array}{l}5.53 \\
(32.40)\end{array}$ & $\begin{array}{l}5.67 \\
(33.46)\end{array}$ & $\begin{array}{l}4.16 \\
(21.4 \\
8)\end{array}$ & $\begin{array}{l}-0.23 \\
63 \\
(2.12)\end{array}$ & $\begin{array}{l}-0.50 \\
65 \\
(2.84)\end{array}$ & $\begin{array}{l}-0.48 \\
64 \\
(2.78)\end{array}$ & $\begin{array}{l}5.12 \\
(29 . \\
13)\end{array}$ & $\begin{array}{l}5.12 \\
(29 . \\
09)\end{array}$ & $\begin{array}{l}5.56 \\
(32.5 \\
8)\end{array}$ & $\begin{array}{l}5.60 \\
(32 . \\
9)\end{array}$ \\
\hline$R 13_{a c}$ & $\begin{array}{l}2.92 \\
(10.0 \\
6)\end{array}$ & $\begin{array}{l}2.92 \\
(9.96 \\
)\end{array}$ & $\begin{array}{l}2.93 \\
(10.03)\end{array}$ & $\begin{array}{l}2.96 \\
(10.15)\end{array}$ & $\begin{array}{l}4.42 \\
(23.2 \\
2)\end{array}$ & $\begin{array}{l}-1.72 \\
(4.86)\end{array}$ & $\begin{array}{l}-1.54 \\
(4.09)\end{array}$ & $\begin{array}{l}-1.55 \\
(4.15)\end{array}$ & $\begin{array}{l}2.93 \\
(10 . \\
10)\end{array}$ & $\begin{array}{l}2.93 \\
(10 . \\
11)\end{array}$ & $\begin{array}{l}2.85 \\
(9.42)\end{array}$ & $\begin{array}{l}2.91 \\
(10 . \\
02)\end{array}$ \\
\hline $\begin{array}{l}R 13_{t h} \\
e\end{array}$ & $\begin{array}{l}4.60 \\
(24.8 \\
5)\end{array}$ & $\begin{array}{l}4.34 \\
(22.3 \\
7)\end{array}$ & $\begin{array}{l}4.24 \\
(21.46)\end{array}$ & $\begin{array}{l}3.06 \\
(14.17)\end{array}$ & $\begin{array}{l}5.24 \\
(30.1 \\
5)\end{array}$ & $\begin{array}{l}-0.37 \\
9 \\
(3.27)\end{array}$ & $\begin{array}{l}0.669 \\
9 \\
(5.33)\end{array}$ & $\begin{array}{l}0.704 \\
3 \\
(5.44)\end{array}$ & $\begin{array}{l}4.41 \\
(23 . \\
24)\end{array}$ & $\begin{array}{l}4.43 \\
(23 . \\
37)\end{array}$ & $\begin{array}{l}4.15 \\
(20.9 \\
5)\end{array}$ & $\begin{array}{l}4.60 \\
(24 . \\
84)\end{array}$ \\
\hline $\begin{array}{l}R 10 a \\
c t_{1}\end{array}$ & $\begin{array}{l}5.23 \\
(29.7 \\
5)\end{array}$ & $\begin{array}{l}5.38 \\
(30.9 \\
4)\end{array}$ & $\begin{array}{l}5.38 \\
(30.96)\end{array}$ & $\begin{array}{l}5.56 \\
(32.54)\end{array}$ & $\begin{array}{l}4.21 \\
(21.2 \\
0)\end{array}$ & $\begin{array}{l}-2.73 \\
(10.1 \\
5)\end{array}$ & $\begin{array}{l}-2.52 \\
(8.84)\end{array}$ & $\begin{array}{l}-2.55 \\
(9.00)\end{array}$ & $\begin{array}{l}5.20 \\
(29 . \\
60)\end{array}$ & $\begin{array}{l}5.18 \\
(29 . \\
42)\end{array}$ & $\begin{array}{l}5.27 \\
(30.3 \\
1)\end{array}$ & $\begin{array}{l}5.23 \\
(29 . \\
78)\end{array}$ \\
\hline $\begin{array}{l}R 10 t \\
h e_{1}\end{array}$ & $\begin{array}{l}3.42 \\
(15.2 \\
1)\end{array}$ & $\begin{array}{l}2.45 \\
(8.00 \\
)\end{array}$ & $\begin{array}{l}2.58 \\
(8.84)\end{array}$ & $\begin{array}{l}3.13 \\
(13.02)\end{array}$ & $\begin{array}{l}4.67 \\
(25.6 \\
2)\end{array}$ & $\begin{array}{l}0.004 \\
2 \\
(2.15)\end{array}$ & $\begin{array}{l}-0.94 \\
99 \\
(6.57)\end{array}$ & $\begin{array}{l}-0.90 \\
41 \\
(6.41)\end{array}$ & $\begin{array}{l}2.25 \\
(8.1 \\
8)\end{array}$ & $\begin{array}{l}1.43 \\
(4.8 \\
4)\end{array}$ & $\begin{array}{l}-0.48 \\
06 \\
(2.92)\end{array}$ & $\begin{array}{l}1.72 \\
(6.5 \\
2)\end{array}$ \\
\hline $\begin{array}{l}R 10 a \\
c t_{2}\end{array}$ & $\begin{array}{l}3.87 \\
(18.1 \\
2)\end{array}$ & $\begin{array}{l}5.30 \\
(30.4 \\
6)\end{array}$ & $\begin{array}{l}5.39 \\
(31.20)\end{array}$ & $\begin{array}{l}4.98 \\
(27.79)\end{array}$ & $\begin{array}{l}3.29 \\
(13.6 \\
2)\end{array}$ & $\begin{array}{l}-2.42 \\
(8.47)\end{array}$ & $\begin{array}{l}-2.07 \\
(6.41)\end{array}$ & $\begin{array}{l}-2.08 \\
(6.43)\end{array}$ & $\begin{array}{l}3.74 \\
(17 . \\
09)\end{array}$ & $\begin{array}{l}3.74 \\
(17 . \\
08)\end{array}$ & $\begin{array}{l}3.87 \\
(18.1 \\
3)\end{array}$ & $\begin{array}{l}3.87 \\
(18 . \\
11)\end{array}$ \\
\hline $\begin{array}{l}R 10 t \\
h e_{2}\end{array}$ & $\begin{array}{l}2.52 \\
(9.55 \\
)\end{array}$ & $\begin{array}{l}2.76 \\
(9.53 \\
)\end{array}$ & $\begin{array}{l}2.91 \\
(11.36)\end{array}$ & $\begin{array}{l}3.96 \\
(18.82)\end{array}$ & $\begin{array}{l}1.69 \\
(4.46 \\
)\end{array}$ & $\begin{array}{l}0.499 \\
5 \\
(2.76)\end{array}$ & $\begin{array}{l}0.512 \\
2 \\
(2.56)\end{array}$ & $\begin{array}{l}0.51 \\
(2.56)\end{array}$ & $\begin{array}{l}2.70 \\
(10 . \\
71)\end{array}$ & $\begin{array}{l}2.70 \\
(10 . \\
71)\end{array}$ & $\begin{array}{l}3.03 \\
(13.5 \\
9)\end{array}$ & $\begin{array}{l}2.52 \\
(9.6 \\
7)\end{array}$ \\
\hline $116_{a c t}$ & $\begin{array}{l}2.63 \\
(9.49 \\
)\end{array}$ & $\begin{array}{l}2.81 \\
(10.4 \\
6)\end{array}$ & $\begin{array}{l}2.80 \\
(10.27)\end{array}$ & $\begin{array}{l}2.92 \\
(12.09)\end{array}$ & $\begin{array}{l}1.75 \\
(5.04 \\
)\end{array}$ & $\begin{array}{l}-0.64 \\
49 \\
(2.80)\end{array}$ & $\begin{array}{l}-0.65 \\
46 \\
(2.59)\end{array}$ & $\begin{array}{l}-0.65 \\
(2.59)\end{array}$ & $\begin{array}{l}2.62 \\
(9.5 \\
3)\end{array}$ & $\begin{array}{l}2.63 \\
(9.6 \\
1)\end{array}$ & $\begin{array}{l}2.74 \\
(10.3 \\
7)\end{array}$ & $\begin{array}{l}2.69 \\
(9.8 \\
0)\end{array}$ \\
\hline $116_{\text {the }}$ & $\begin{array}{l}5.53 \\
(32.4 \\
0)\end{array}$ & $\begin{array}{l}5.55 \\
(32.5 \\
1)\end{array}$ & $\begin{array}{l}5.50 \\
(32.15)\end{array}$ & $\begin{array}{l}1.11 \\
(3.33)\end{array}$ & $\begin{array}{l}5.33 \\
(30.6 \\
9)\end{array}$ & $\begin{array}{l}-0.36 \\
6 \\
(2.06)\end{array}$ & $\begin{array}{l}1.90 \\
(9.91)\end{array}$ & $\begin{array}{l}1.97 \\
(10.2 \\
5)\end{array}$ & $\begin{array}{l}5.49 \\
(32 . \\
13)\end{array}$ & $\begin{array}{l}5.50 \\
(32 . \\
16)\end{array}$ & $\begin{array}{l}5.37 \\
(31.1 \\
9)\end{array}$ & $\begin{array}{l}5.54 \\
(32 . \\
44)\end{array}$ \\
\hline $\begin{array}{l}C 14 a \\
c t_{1}\end{array}$ & $\begin{array}{l}3.57 \\
(16.0 \\
4)\end{array}$ & $\begin{array}{l}0.32 \\
79 \\
(2.67 \\
)\end{array}$ & $\begin{array}{l}0.2817 \\
(2.83)\end{array}$ & $\begin{array}{l}0.2869 \\
(2.83)\end{array}$ & $\begin{array}{l}3.89 \\
(17.6 \\
4)\end{array}$ & $\begin{array}{l}4.03 \\
(19.3 \\
8)\end{array}$ & $\begin{array}{l}4.03 \\
(19.3 \\
8)\end{array}$ & $\begin{array}{l}3.87 \\
(20.2 \\
1)\end{array}$ & $\begin{array}{l}3.86 \\
(17 . \\
48)\end{array}$ & $\begin{array}{l}3.87 \\
(17 . \\
58)\end{array}$ & $\begin{array}{l}3.81 \\
(17.2 \\
5)\end{array}$ & $\begin{array}{l}3.90 \\
(17 . \\
70)\end{array}$ \\
\hline $\begin{array}{l}C 14 t \\
h e_{1}\end{array}$ & $\begin{array}{l}2.59 \\
(5.72 \\
)\end{array}$ & $\begin{array}{l}3.11 \\
(2.25 \\
)\end{array}$ & $\begin{array}{l}2.97 \\
(2.31)\end{array}$ & $\begin{array}{l}2.63 \\
(2.32)\end{array}$ & $\begin{array}{l}1.92 \\
(9.95 \\
)\end{array}$ & $\begin{array}{l}-0.45 \\
75 \\
(12.6 \\
9)\end{array}$ & $\begin{array}{l}-0.32 \\
32 \\
(11.8 \\
3)\end{array}$ & $\begin{array}{l}-0.31 \\
9 \\
(10.5 \\
7)\end{array}$ & $\begin{array}{l}2.71 \\
(10 . \\
56)\end{array}$ & $\begin{array}{l}2.71 \\
(10 . \\
59)\end{array}$ & $\begin{array}{l}3.70 \\
(18.3 \\
3)\end{array}$ & $\begin{array}{l}2.94 \\
(12 . \\
36)\end{array}$ \\
\hline $\begin{array}{l}C 14 a \\
c t_{2}\end{array}$ & $\begin{array}{l}5.42 \\
(32.3 \\
4)\end{array}$ & $\begin{array}{l}1.83 \\
(30.8 \\
)\end{array}$ & $\begin{array}{l}-0.26 \\
79 \\
(30.76)\end{array}$ & $\begin{array}{l}-0.26 \\
33 \\
(13.05)\end{array}$ & $\begin{array}{l}5.53 \\
(31.4 \\
7)\end{array}$ & $\begin{array}{l}5.34 \\
(9.25)\end{array}$ & $\begin{array}{l}5.34 \\
(2.68)\end{array}$ & $\begin{array}{l}3.12 \\
(2.63)\end{array}$ & $\begin{array}{l}5.49 \\
(32 . \\
01)\end{array}$ & $\begin{array}{l}5.49 \\
(32 . \\
03)\end{array}$ & $\begin{array}{l}5.31 \\
(30.5 \\
3)\end{array}$ & $\begin{array}{l}5.50 \\
(32 . \\
14)\end{array}$ \\
\hline $\begin{array}{l}C 14 t \\
h e_{2}\end{array}$ & $\begin{array}{l}3.07 \\
(12.3 \\
4)\end{array}$ & $\begin{array}{l}3.31 \\
(13.3 \\
5)\end{array}$ & $\begin{array}{l}3.28 \\
(13.3)\end{array}$ & $\begin{array}{l}3.43 \\
(15.28)\end{array}$ & $\begin{array}{l}2.50 \\
(8.65 \\
)\end{array}$ & $\begin{array}{l}-0.40 \\
76 \\
(2.22)\end{array}$ & $\begin{array}{l}-0.01 \\
95 \\
(2.09)\end{array}$ & $\begin{array}{l}-0.01 \\
43 \\
(2.10)\end{array}$ & $\begin{array}{l}3.16 \\
(12 . \\
84)\end{array}$ & $\begin{array}{l}3.17 \\
(12 . \\
87)\end{array}$ & $\begin{array}{l}4.20 \\
(21.4 \\
8)\end{array}$ & $\begin{array}{l}3.44 \\
(15 . \\
12)\end{array}$ \\
\hline$C 15_{a c}$ & $\begin{array}{l}4.41 \\
(21.5\end{array}$ & $\begin{array}{l}5.21 \\
(29.3\end{array}$ & $\begin{array}{l}5.25 \\
(29.78)\end{array}$ & $\begin{array}{l}5.75 \\
(34.03)\end{array}$ & $\begin{array}{l}3.85 \\
(15.9\end{array}$ & $\begin{array}{l}1.72 \\
(12.9)\end{array}$ & $\begin{array}{l}4.23 \\
(24.2\end{array}$ & $\begin{array}{l}4.32 \\
(24.5\end{array}$ & $\begin{array}{l}5.74 \\
(33 .\end{array}$ & $\begin{array}{l}5.74 \\
(33 .\end{array}$ & $\begin{array}{l}3.98 \\
(17.8\end{array}$ & $\begin{array}{l}5.74 \\
(33 .\end{array}$ \\
\hline
\end{tabular}




\begin{tabular}{|c|c|c|c|c|c|c|c|c|c|c|c|c|}
\hline & $\begin{array}{l}R V_{t} \\
m\end{array}$ & $\begin{array}{l}B P V t \\
(m)\end{array}$ & $\begin{array}{l}R V_{t m a} \\
\left.a f^{\prime \prime}\right)\end{array}$ & $\begin{array}{l}R V_{t m a} \\
a d^{(2)}\end{array}$ & $\begin{array}{l}M R_{t} \\
\text { Part }\end{array}$ & $\begin{array}{l}M R_{t G} \\
\kappa\end{array}$ & $\begin{array}{l}M R_{t R} \\
S\end{array}$ & $\begin{array}{l}M R t Y \\
Z\end{array}$ & $\begin{array}{l}R R_{t} \\
\text { Part }\end{array}$ & $\begin{array}{l}R R_{t} \\
G K\end{array}$ & $\begin{array}{l}R R_{t} R \\
S\end{array}$ & $\begin{array}{l}R R_{t} \\
Y Z\end{array}$ \\
\hline & 9) & 7) & & & 8) & & 4) & 4) & 99) & 99) & 6) & 98) \\
\hline$C 15_{t h}$ & $\begin{array}{l}5.75 \\
(34.0 \\
3)\end{array}$ & $\begin{array}{l}5.75 \\
(34.0 \\
3)\end{array}$ & $\begin{array}{l}5.75 \\
(34.03)\end{array}$ & $\begin{array}{l}5.75 \\
(34.03)\end{array}$ & $\begin{array}{l}5.74 \\
(34.0 \\
0)\end{array}$ & $\begin{array}{l}-2.72 \\
(13.3 \\
1)\end{array}$ & $\begin{array}{l}4.99 \\
(28.5 \\
8)\end{array}$ & $\begin{array}{l}5.08 \\
(29.2 \\
1)\end{array}$ & $\begin{array}{l}5.75 \\
(34 . \\
03)\end{array}$ & $\begin{array}{l}5.75 \\
(34 . \\
03)\end{array}$ & $\begin{array}{l}2.52 \\
(8.20)\end{array}$ & $\begin{array}{l}5.75 \\
(34 . \\
03)\end{array}$ \\
\hline
\end{tabular}

Notes. Table 4 reports the descriptive statistics of skewness (outside brackets) and kurtosis (within brackets) for the risk estimates of both actual and theoretical BRIC Eurobond prices. Risk estimates are split into three groups: realized volatility, monthly range-based, and realized range estimates.

Table 5.

Risk-descriptive statistics-CVM and $Q Q$ tests.

\begin{tabular}{|c|c|c|c|c|c|c|c|c|c|c|c|c|}
\hline & $R V_{t m}$ & $\begin{array}{l}B P V_{t} \\
m\end{array}$ & $\begin{array}{l}R V_{t m a} \\
a d^{\prime \prime)}\end{array}$ & $\begin{array}{l}R V_{t m a} \\
a d / 2)\end{array}$ & $\begin{array}{l}\text { MRtP } \\
a r\end{array}$ & $\begin{array}{l}M R_{t} \\
G K\end{array}$ & $\begin{array}{l}M R_{t} \\
R S\end{array}$ & $\begin{array}{l}M R t Y \\
Z\end{array}$ & $\begin{array}{l}R R_{t P} \\
a r\end{array}$ & $\begin{array}{l}R R_{t G} \\
\kappa\end{array}$ & $\begin{array}{l}R R t \\
R S\end{array}$ & $\begin{array}{l}R R t Y \\
Z\end{array}$ \\
\hline $\begin{array}{l}B 14_{a} \\
a t\end{array}$ & $\begin{array}{l}2.02 \square \\
(3.29 \\
)\end{array}$ & $\begin{array}{l}2.14 \square \\
(2.44 \\
)\end{array}$ & $\begin{array}{l}2.14 \square \\
(2.43)\end{array}$ & $\begin{array}{l}2.17 \square \\
(2.77)\end{array}$ & $\begin{array}{l}2.12 \square \\
(3.79)\end{array}$ & $\begin{array}{l}0.35 \\
87 \\
(52.6 \\
2 \square)\end{array}$ & $\begin{array}{l}0.49 \\
24 \square \\
(44.5 \\
8 \square)\end{array}$ & $\begin{array}{l}0.482 \\
9 \square \\
(45.2 \\
6 \square)\end{array}$ & $\begin{array}{l}2.07 \square \\
(2.94)\end{array}$ & $\begin{array}{l}2.07 \square \\
(2.95)\end{array}$ & $\begin{array}{l}2.16 \\
\square \\
(2.6 \\
5)\end{array}$ & $\begin{array}{l}2.03 \square \\
(3.24)\end{array}$ \\
\hline $\begin{array}{l}B 14_{t h} \\
e\end{array}$ & $\begin{array}{l}1.20 \square \\
(18.2 \\
3)\end{array}$ & $\begin{array}{l}1.47 \square \\
(18.5 \\
4)\end{array}$ & $\begin{array}{l}1.41 \square \\
(18.29)\end{array}$ & $\begin{array}{l}1.37 \square \\
(7.97)\end{array}$ & $\begin{array}{l}1.13 \square \\
(28.9 \\
2)\end{array}$ & $\begin{array}{l}0.06 \\
34 \\
(28.7 \\
9)\end{array}$ & $\begin{array}{l}0.12 \\
10 \\
(16.6 \\
8)\end{array}$ & $\begin{array}{l}0.124 \\
3 \\
(16.7 \\
7)\end{array}$ & $\begin{array}{l}1.21 \square \\
(18.2 \\
4)\end{array}$ & $\begin{array}{l}1.21 \square \\
(18.2 \\
6)\end{array}$ & $\begin{array}{l}1.37 \\
\square \\
(13 . \\
95)\end{array}$ & $\begin{array}{l}1.28 \square \\
(17.4 \\
2)\end{array}$ \\
\hline $\begin{array}{l}B 11_{a} \\
a t\end{array}$ & $\begin{array}{l}1.48 \unrhd \\
(5.33 \\
)\end{array}$ & $\begin{array}{l}1.59 \square \\
(4.81 \\
)\end{array}$ & $\begin{array}{l}1.60 \square \\
(4.71)\end{array}$ & $\begin{array}{l}1.72 \square \\
(4.65)\end{array}$ & $\begin{array}{l}1.53 \square \\
(6.08)\end{array}$ & $\begin{array}{l}0.09 \\
83 \\
(47.6 \\
\left.9_{\square}\right)\end{array}$ & $\begin{array}{l}0.17 \\
57 \\
(39.3 \\
6 \square)\end{array}$ & $\begin{array}{l}0.173 \\
0 \\
(39.5 \\
2 \unrhd)\end{array}$ & $\begin{array}{c}1.47 \square \\
(5.05)\end{array}$ & $\begin{array}{l}0.382 \\
3 \\
(27.2 \\
8)\end{array}$ & $\begin{array}{l}1.40 \\
\square \\
(12 . \\
78)\end{array}$ & $\begin{array}{l}1.21 \square \\
(13.0 \\
9)\end{array}$ \\
\hline $\begin{array}{l}B 11_{\text {th }} \\
e\end{array}$ & $\begin{array}{l}0.539 \\
8 \square \\
(27.1 \\
2)\end{array}$ & $\begin{array}{l}0.546 \\
0 \square \\
(18.9 \\
4)\end{array}$ & $\begin{array}{l}0.5384 \\
\square(16.7)\end{array}$ & $\begin{array}{l}0.6118 \\
\square \\
(19.63)\end{array}$ & $\begin{array}{l}0.557 \\
4 \square \\
(54.7 \\
4 \square)\end{array}$ & $\begin{array}{l}0.04 \\
17 \\
(24.9 \\
5)\end{array}$ & $\begin{array}{l}0.03 \\
90 \\
(51.5 \\
2 \square)\end{array}$ & $\begin{array}{l}0.039 \\
0 \\
(51.3 \\
5 \square)\end{array}$ & $\begin{array}{l}1.18 \square \\
(19.0 \\
6)\end{array}$ & $\begin{array}{l}0.485 \\
6 \square \\
(15.7 \\
3)\end{array}$ & $\begin{array}{l}0.80 \\
90 \square \\
(30 . \\
14)\end{array}$ & $\begin{array}{l}0.841 \\
5 \square \\
(31.1 \\
5)\end{array}$ \\
\hline $\begin{array}{l}B 12 a \\
a t\end{array}$ & $\begin{array}{l}1.63 \unrhd \\
(6.04 \\
)\end{array}$ & $\begin{array}{l}1.70 \square \\
(4.51 \\
)\end{array}$ & $\begin{array}{l}1.69 \square \\
(4.53)\end{array}$ & $\begin{array}{l}2.04 \square \\
(4.18)\end{array}$ & $\begin{array}{l}1.82 \square \\
(5.63)\end{array}$ & $\begin{array}{l}0.13 \\
07 \\
(40.0 \\
1 \square)\end{array}$ & $\begin{array}{l}0.19 \\
41 \\
(53.2 \\
8 \square)\end{array}$ & $\begin{array}{l}0.191 \\
2 \\
(53.6 \\
6 \unrhd)\end{array}$ & $\begin{array}{l}1.72 \square \\
(5.10)\end{array}$ & $\begin{array}{l}1.72 \square \\
(5.14)\end{array}$ & $\begin{array}{l}1.94 \\
\square \\
(3.4 \\
6)\end{array}$ & $\begin{array}{l}1.65 \square \\
(5.91)\end{array}$ \\
\hline $\begin{array}{l}B 12_{\text {th }} \\
e\end{array}$ & $\begin{array}{l}1.67 \square \\
(4.55 \\
)\end{array}$ & $\begin{array}{l}2.02 \square \\
(2.18 \\
)\end{array}$ & $\begin{array}{l}2.04 \square \\
(2.10)\end{array}$ & $\begin{array}{c}2.31_{\square} \\
(1.20)\end{array}$ & $\begin{array}{l}1.28 \square \\
(8.59)\end{array}$ & $\begin{array}{l}0.06 \\
58 \\
(93.3 \\
6 \sqsupseteq)\end{array}$ & $\begin{array}{l}0.03 \\
92 \\
(27.2 \\
5)\end{array}$ & $\begin{array}{l}0.039 \\
0 \\
(27.6 \\
8)\end{array}$ & $\begin{array}{l}1.65 \square \\
(4.57)\end{array}$ & $\begin{array}{l}1.65 \square \\
(4.61)\end{array}$ & $\begin{array}{l}2.09 \\
\square \\
(2.0 \\
1)\end{array}$ & $\begin{array}{l}2.19 \\
(1.70)\end{array}$ \\
\hline $\begin{array}{l}R 13_{a} \\
c t\end{array}$ & $\begin{array}{l}1.76 \square \\
(36.0 \\
7)\end{array}$ & $\begin{array}{l}1.92 \square \\
(32.6 \\
)\end{array}$ & $\begin{array}{l}1.92 \square \\
(32.29)\end{array}$ & $\begin{array}{l}1.84 \square \\
(33.77)\end{array}$ & $\begin{array}{l}1.72 \square \\
(18.1 \\
5)\end{array}$ & $\begin{array}{l}0.82 \\
13 \square \\
(124 . \\
67 \unrhd)\end{array}$ & $\begin{array}{l}0.76 \\
48 \unrhd \\
(129 . \\
35 \unrhd)\end{array}$ & $\begin{array}{l}0.761 \\
9 \square \\
(128 . \\
22 \square)\end{array}$ & $\begin{array}{l}1.79 \square \\
(35.8 \\
6)\end{array}$ & $\begin{array}{l}1.79 \square \\
(35.8 \\
5)\end{array}$ & $\begin{array}{l}1.79 \\
\square \\
(35 . \\
60)\end{array}$ & $\begin{array}{l}1.76 \square \\
(36.1 \\
6)\end{array}$ \\
\hline $\begin{array}{l}R 13_{t h} \\
e^{\prime}\end{array}$ & $\begin{array}{l}1.31 \square \\
(8.92 \\
)\end{array}$ & $\begin{array}{l}1.38 \square \\
(11.2 \\
7)\end{array}$ & $\begin{array}{l}1.38 \unrhd \\
(11.77)\end{array}$ & $\begin{array}{l}0.5907 \\
\square \\
(15.56)\end{array}$ & $\begin{array}{l}1.46 \square \\
(4.25)\end{array}$ & $\begin{array}{l}0.04 \\
35 \\
(34.1 \\
0)\end{array}$ & $\begin{array}{l}0.09 \\
39 \\
(15.9 \\
5)\end{array}$ & $\begin{array}{l}0.096 \\
3 \\
(15.7 \\
9)\end{array}$ & $\begin{array}{l}1.26 \square \\
(10.3 \\
1)\end{array}$ & $\begin{array}{l}1.27 \unrhd \\
(10.1 \\
5)\end{array}$ & $\begin{array}{l}1.21 \\
\square \\
(12 . \\
38)\end{array}$ & $\begin{array}{l}1.31 \square \\
(8.84)\end{array}$ \\
\hline $\begin{array}{l}R 10 a \\
c t_{1}\end{array}$ & $\begin{array}{l}1.99 \square \\
(6.98\end{array}$ & $\begin{array}{l}1.10 \unrhd \\
(4.25\end{array}$ & $\begin{array}{l}1.00 \square \\
(4.25)\end{array}$ & $\begin{array}{l}0.9950 \\
\square(3.67)\end{array}$ & $\begin{array}{l}2.14 \square \\
(17.4\end{array}$ & $\begin{array}{l}2.36 \\
\square\end{array}$ & $\begin{array}{l}2.37 \\
\square\end{array}$ & $\begin{array}{l}2.31 \square \\
(54.7\end{array}$ & $\begin{array}{l}2.11 \square \\
(7.46)\end{array}$ & $\begin{array}{l}1.97 \square \\
(7.08)\end{array}$ & $\begin{array}{l}1.85 \\
\square\end{array}$ & $\begin{array}{l}2.09 \square \\
(7.04)\end{array}$ \\
\hline
\end{tabular}




\begin{tabular}{|c|c|c|c|c|c|c|c|c|c|c|c|c|}
\hline & $R V_{t m}$ & $\begin{array}{l}B P V_{t} \\
m\end{array}$ & $\begin{array}{l}R V t m a \\
\left.a d j^{\prime \prime}\right)\end{array}$ & $\begin{array}{l}R V_{t m a} \\
\left.a d p^{2}\right)\end{array}$ & $\begin{array}{l}M R_{t} P \\
a r\end{array}$ & $\begin{array}{l}M R_{t} \\
G K\end{array}$ & $\begin{array}{l}M R_{t} \\
R S\end{array}$ & $\begin{array}{l}M R_{t} Y \\
Z\end{array}$ & $\begin{array}{l}R R_{t} P \\
a r\end{array}$ & $\begin{array}{l}R R_{t} G \\
k\end{array}$ & $\begin{array}{l}R R t \\
R S\end{array}$ & $\begin{array}{l}R R_{t} Y \\
Z\end{array}$ \\
\hline & ) & ) & & & 1) & $\begin{array}{l}(54.0 \\
7 \text { ㅁ) }\end{array}$ & $\begin{array}{l}(55.8 \\
3 \unrhd)\end{array}$ & 1ㅁ) & & & $\begin{array}{l}(5.2 \\
7)\end{array}$ & \\
\hline $\begin{array}{l}R 10 t \\
h e_{1}\end{array}$ & $\begin{array}{l}1.25 \square \\
(14.1 \\
8)\end{array}$ & $\begin{array}{l}1.28 \unrhd \\
(22.7 \\
4)\end{array}$ & $\begin{array}{l}1.26 \unrhd \\
(21.40)\end{array}$ & $\begin{array}{l}1.31 \square \\
(16.33)\end{array}$ & $\begin{array}{l}1.40 \square \\
(5.33)\end{array}$ & $\begin{array}{l}0.04 \\
17 \\
(131 . \\
96 \square)\end{array}$ & $\begin{array}{l}0.06 \\
61 \\
(88.8 \\
8 \square)\end{array}$ & $\begin{array}{l}0.063 \\
6 \\
(89.6 \\
6 \unrhd)\end{array}$ & $\begin{array}{l}0.785 \\
5 \square \\
(20.9 \\
3)\end{array}$ & $\begin{array}{l}0.380 \\
1 \\
(23.5 \\
6)\end{array}$ & $\begin{array}{l}0.30 \\
74 \\
(30 . \\
09)\end{array}$ & $\begin{array}{l}0.667 \\
4 \square \\
(24.7 \\
7)\end{array}$ \\
\hline $\begin{array}{l}R 10 a \\
c t_{2}\end{array}$ & $\begin{array}{l}1.99 \square \\
(17.5 \\
9)\end{array}$ & $\begin{array}{l}2.08 \square \\
(4.64 \\
)\end{array}$ & $\begin{array}{l}2.15 \square \\
(3.80)\end{array}$ & $\begin{array}{l}2.13 \square \\
(6.52)\end{array}$ & $\begin{array}{l}1.98 \unrhd \\
(26.2 \\
2)\end{array}$ & $\begin{array}{l}0.80 \\
76 \square \\
(74.9 \\
7 \unrhd)\end{array}$ & $\begin{array}{l}0.75 \\
47 \square \\
(84.3 \\
2 \square)\end{array}$ & $\begin{array}{l}0.750 \\
8 \square \\
(84.1 \\
4 \square)\end{array}$ & $\begin{array}{l}1.98 \square \\
(19.5 \\
9)\end{array}$ & $\begin{array}{l}1.98 \square \\
(19.6 \\
0)\end{array}$ & $\begin{array}{l}1.99 \\
\square \\
(17 . \\
55)\end{array}$ & $\begin{array}{l}1.99 \square \\
(17.6 \\
0)\end{array}$ \\
\hline $\begin{array}{l}R 10 t \\
h e_{2}\end{array}$ & $\begin{array}{l}0.878 \\
4 \square \\
(10.9 \\
5)\end{array}$ & $\begin{array}{l}1.29 \square \\
(13.4 \\
4)\end{array}$ & $\begin{array}{l}1.13 \square \\
(13.80)\end{array}$ & $\begin{array}{l}1.46 \square \\
(12.82)\end{array}$ & $\begin{array}{l}0.937 \\
9 \square \\
(15.0 \\
9)\end{array}$ & $\begin{array}{l}0.05 \\
32 \\
(34.1 \\
8)\end{array}$ & $\begin{array}{l}0.06 \\
85 \\
(52.4 \\
8 \unrhd)\end{array}$ & $\begin{array}{l}0.068 \\
7 \\
(52.3 \\
0 \sqsubseteq)\end{array}$ & $\begin{array}{l}0.886 \\
7 \square \\
(11.9 \\
0)\end{array}$ & $\begin{array}{l}0.886 \\
4 \square \\
(11.9 \\
6)\end{array}$ & $\begin{array}{l}0.80 \\
51 \square \\
(11 . \\
28)\end{array}$ & $\begin{array}{l}0.832 \\
6 \square \\
(11.1 \\
7)\end{array}$ \\
\hline 116 & $\begin{array}{l}0.843 \\
5 \square \\
(20.1 \\
3)\end{array}$ & $\begin{array}{l}1.01 \square \\
(14.4 \\
0)\end{array}$ & $\begin{array}{l}1.04 \unrhd \\
(14.60)\end{array}$ & $\begin{array}{l}0.7566 \\
\square \\
(17.01)\end{array}$ & $\begin{array}{l}0.624 \\
3 \unrhd \\
(27.9 \\
0)\end{array}$ & $\begin{array}{l}0.18 \\
06 \\
(169 . \\
59 \unrhd)\end{array}$ & $\begin{array}{l}0.19 \\
97 \\
(134 . \\
23 \unrhd)\end{array}$ & $\begin{array}{l}0.198 \\
6 \\
(134 . \\
28 \unrhd)\end{array}$ & $\begin{array}{l}0.818 \\
9 \unrhd \\
(20.4 \\
2)\end{array}$ & $\begin{array}{l}0.822 \\
9 \square \\
(20.3 \\
0)\end{array}$ & $\begin{array}{l}0.93 \\
82 \square \\
(18 . \\
45)\end{array}$ & $\begin{array}{l}0.885 \\
7 \square \\
(19.6 \\
7)\end{array}$ \\
\hline $116_{\text {the }}$ & $\begin{array}{l}1.87 \square \\
(1.61 \\
)\end{array}$ & $\begin{array}{l}1.94 \square \\
(1.68 \\
)\end{array}$ & $\begin{array}{l}1.85 \square \\
(2.04)\end{array}$ & $\begin{array}{l}0.3783 \\
(48.79 \\
\text { ㅁ) }\end{array}$ & $\begin{array}{l}1.84 \square \\
(2.30)\end{array}$ & $\begin{array}{l}0.06 \\
47 \\
(34.1 \\
0)\end{array}$ & $\begin{array}{l}0.13 \\
65 \\
(14.0 \\
6)\end{array}$ & $\begin{array}{l}0.142 \\
9 \\
(13.8 \\
1)\end{array}$ & $\begin{array}{l}1.80 \square \\
(1.79)\end{array}$ & $\begin{array}{c}1.81 \square \\
(1.77)\end{array}$ & $\begin{array}{l}1.66 \\
\square \\
(2.6 \\
0)\end{array}$ & $\begin{array}{l}.89 \square \\
1.55)\end{array}$ \\
\hline $\begin{array}{l}C 14 a \\
c t_{1}\end{array}$ & $\begin{array}{l}1.49 \square \\
(11.7 \\
4)\end{array}$ & $\begin{array}{l}1.26 \square \\
(8.96 \\
)\end{array}$ & $\begin{array}{l}1.25 \square \\
(8.88)\end{array}$ & $\begin{array}{l}0.5929 \\
\square \\
(15.15)\end{array}$ & $\begin{array}{l}1.26 \square \\
(14.7 \\
8)\end{array}$ & $\begin{array}{l}0.05 \\
73 \\
(64.5 \\
8 \unrhd)\end{array}$ & $\begin{array}{l}0.02 \\
51 \\
(63.1 \\
7 \square)\end{array}$ & $\begin{array}{l}0.025 \\
8 \\
(63.7 \\
3 \unrhd)\end{array}$ & $\begin{array}{l}1.42 \square \\
(12.1 \\
7)\end{array}$ & $\begin{array}{l}1.43 \square \\
(12.0 \\
9)\end{array}$ & $\begin{array}{l}1.31 \\
\square \\
(11 . \\
42)\end{array}$ & $\begin{array}{l}1.48 \square \\
(11.6 \\
3)\end{array}$ \\
\hline $\begin{array}{l}C 14 t \\
h e_{1}\end{array}$ & $\begin{array}{l}0.669 \\
0 \square \\
(17.9 \\
2)\end{array}$ & $\begin{array}{l}0.941 \\
4 \square \\
(12.1 \\
4)\end{array}$ & $\begin{array}{l}0.9114 \\
\square \\
(12.65)\end{array}$ & $\begin{array}{l}0.7668 \\
\square \\
(17.31)\end{array}$ & $\begin{array}{l}0.697 \\
0 \square \\
(28.3 \\
9)\end{array}$ & $\begin{array}{l}0.07 \\
54 \square \\
(53.1 \\
6 \unrhd)\end{array}$ & $\begin{array}{l}0.12 \\
73 \\
(38.2 \\
2 \square)\end{array}$ & $\begin{array}{l}0.126 \\
2 \\
(38.2 \\
2 \square)\end{array}$ & $\begin{array}{l}1.00 \square \\
(12.1 \\
5)\end{array}$ & $\begin{array}{l}1.00 \square \\
(12.0 \\
6)\end{array}$ & $\begin{array}{l}1.25 \\
\square \\
(7.1 \\
7)\end{array}$ & $\begin{array}{l}1.05 \square \\
(10.6 \\
9)\end{array}$ \\
\hline $\begin{array}{l}C 14 a \\
c t_{2}\end{array}$ & $\begin{array}{l}2.03 \square \\
(1.82 \\
)\end{array}$ & $\begin{array}{l}0.111 \\
9 \\
(3.21 \\
)\end{array}$ & $\begin{array}{l}0.0352 \\
(3.24)\end{array}$ & $\begin{array}{l}0.0382 \\
(18.13)\end{array}$ & $\begin{array}{l}2.11 \square \\
(2.43)\end{array}$ & $\begin{array}{l}2.03 \\
\square \\
(41.1 \\
0 \square)\end{array}$ & $\begin{array}{l}2.02 \\
\square \\
(45.9 \\
9 \square)\end{array}$ & $\begin{array}{l}0.992 \\
1 \square \\
(47.2 \\
3 \unrhd)\end{array}$ & $\begin{array}{l}2.07 \unrhd \\
(2.07)\end{array}$ & $\begin{array}{l}2.07 \sqsubseteq \\
(2.05)\end{array}$ & $\begin{array}{l}2.01 \\
\square \\
(3.5 \\
0)\end{array}$ & $\begin{array}{l}2.09 \square \\
(2.00)\end{array}$ \\
\hline $\begin{array}{l}C 14 t \\
h e_{2}\end{array}$ & $\begin{array}{l}0.950 \\
7 \square \\
(12.5 \\
6)\end{array}$ & $\begin{array}{l}1.20 \square \\
(10.3 \\
2)\end{array}$ & $\begin{array}{l}1.16 \unrhd \\
(10.22)\end{array}$ & $\begin{array}{l}1.06 \unrhd \\
(11.22)\end{array}$ & $\begin{array}{l}0.833 \\
8 \square \\
(28.3 \\
0)\end{array}$ & $\begin{array}{l}0.10 \\
27 \square \\
(52.5 \\
9 \unrhd)\end{array}$ & $\begin{array}{l}0.03 \\
78 \\
(29.9 \\
4)\end{array}$ & $\begin{array}{l}0.037 \\
1 \\
(29.9 \\
3)\end{array}$ & $\begin{array}{l}0.709 \\
1 \square \\
(17.0 \\
2)\end{array}$ & $\begin{array}{l}0.712 \\
6 \square \\
(16.8 \\
8)\end{array}$ & $\begin{array}{l}0.88 \\
82 \square \\
(9.1 \\
2)\end{array}$ & $\begin{array}{l}0.741 \\
\square \\
(14.3 \\
5)\end{array}$ \\
\hline$c 15 a$ & $\begin{array}{l}2.58 \square \\
(10.3 \\
9)\end{array}$ & $\begin{array}{l}2.58 \square \\
(10.3 \\
7)\end{array}$ & $\begin{array}{l}2.58 \unrhd \\
(7.01)\end{array}$ & $\begin{array}{l}2.82 \square \\
(7.53)\end{array}$ & $\begin{array}{l}2.41 \square \\
(7.24)\end{array}$ & $\begin{array}{l}0.59 \\
93 \square \\
(2.94 \\
)\end{array}$ & $\begin{array}{l}0.97 \\
53 \square \\
(2.71 \\
)\end{array}$ & $\begin{array}{l}0.978 \\
8 \square \\
(0.47 \\
44)\end{array}$ & $\begin{array}{l}2.76 \square \\
(0.46 \\
48)\end{array}$ & $\begin{array}{l}2.76 \square \\
(0.46 \\
48)\end{array}$ & $\begin{array}{l}1.96 \\
\square \\
(17 . \\
28)\end{array}$ & $\begin{array}{l}2.76 \square \\
(0.46 \\
77)\end{array}$ \\
\hline$C 15_{t h}$ & $\begin{array}{l}2.81 \square \\
(0.68 \\
57)\end{array}$ & $\begin{array}{l}2.82 \square \\
(0.68 \\
80)\end{array}$ & $\begin{array}{l}2.82 \square \\
(0.687 \\
5)\end{array}$ & $\begin{array}{l}2.81 \square \\
(0.686 \\
8)\end{array}$ & $\begin{array}{l}2.74 \square \\
(0.68 \\
26)\end{array}$ & $\begin{array}{l}0.24 \\
16 \\
(16.5\end{array}$ & $\begin{array}{l}1.12 \\
\square \\
(5.04\end{array}$ & $\begin{array}{l}1.20 \square \\
(4.58)\end{array}$ & $\begin{array}{l}2.85 \square \\
(0.68 \\
29)\end{array}$ & $\begin{array}{l}2.85 \square \\
(0.68 \\
29)\end{array}$ & $\begin{array}{l}1.59 \\
\square \\
(20 .\end{array}$ & $\begin{array}{l}2.85 \square \\
(0.68 \\
2)\end{array}$ \\
\hline
\end{tabular}




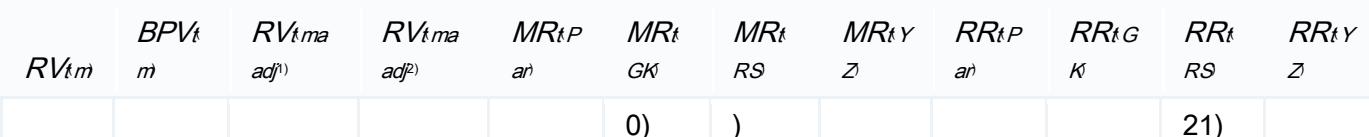

0) )

Notes. Table 5 reports the descriptive statistics of CVM (outside brackets) and QQ (within brackets) normality tests for the risk (volatility) estimates of both actual and theoretical BRIC Eurobond prices. Risk (volatility) estimates are split into three groups: realized volatility, monthly range, and realized range estimates.

Indicates significance in a $5 \%$ significance level.

Table 6.

Average magnitude of risk $\bar{R}$ ).

\begin{tabular}{|c|c|c|c|c|c|c|c|c|c|c|c|c|}
\hline & $R V_{t m}$ & $\begin{array}{l}B P V_{t} \\
m\end{array}$ & $\begin{array}{l}R V_{t m a} \\
a d j^{\prime \prime)}\end{array}$ & $\begin{array}{l}R V_{t m a} \\
\left.a d f^{2}\right)\end{array}$ & $\begin{array}{l}M R_{t P} \\
a r\end{array}$ & $\begin{array}{l}M R_{t} G \\
\kappa\end{array}$ & $\begin{array}{l}M R_{t R} \\
S\end{array}$ & $\begin{array}{l}M R_{t Y} \\
Z\end{array}$ & $\begin{array}{l}R R_{t} P \\
a r\end{array}$ & $\begin{array}{l}R R_{t G} \\
\kappa\end{array}$ & $\begin{array}{l}R R_{t R} \\
S\end{array}$ & $\begin{array}{l}R R A Y \\
Z\end{array}$ \\
\hline $\begin{array}{l}B 14_{a} \\
c t\end{array}$ & $\begin{array}{c}8.54 \mathrm{e} \\
-4\end{array}$ & $\begin{array}{c}9.32 \mathrm{e} \\
-4\end{array}$ & $\begin{array}{l}9.31 \mathrm{e} \\
-4\end{array}$ & $\begin{array}{l}6.12 e \\
-4\end{array}$ & $\begin{array}{l}6.50 \mathrm{e} \\
-4\end{array}$ & $\begin{array}{c}5.39 e \\
-5\end{array}$ & $\begin{array}{c}3.90 \mathrm{e} \\
-5\end{array}$ & $\begin{array}{c}3.60 \mathrm{e} \\
-5\end{array}$ & $\begin{array}{l}5.47 e \\
-4\end{array}$ & $\begin{array}{l}5.58 \mathrm{e} \\
-4\end{array}$ & $\begin{array}{c}4.94 \mathrm{e} \\
-4\end{array}$ & $\begin{array}{c}8.15 e \\
-4\end{array}$ \\
\hline $\begin{array}{l}B 14_{t} \\
h e\end{array}$ & $\begin{array}{l}0.001 \\
3\end{array}$ & $\begin{array}{l}0.002 \\
0\end{array}$ & $\begin{array}{l}0.001 \\
9\end{array}$ & $\begin{array}{l}7.40 \mathrm{e} \\
-4\end{array}$ & $\begin{array}{c}5.49 e \\
-4\end{array}$ & $\begin{array}{c}5.54 \mathrm{e} \\
-5\end{array}$ & $\begin{array}{l}4.02 e \\
-5\end{array}$ & $\begin{array}{c}3.71 \mathrm{e} \\
-5\end{array}$ & $\begin{array}{c}8.18 \mathrm{e} \\
-4\end{array}$ & $\begin{array}{l}8.42 e \\
-4\end{array}$ & $\begin{array}{c}7.36 \mathrm{e} \\
-4\end{array}$ & $\begin{array}{l}0.001 \\
3\end{array}$ \\
\hline $\begin{array}{l}B 11_{a} \\
c t\end{array}$ & $\begin{array}{c}3.06 e \\
-4\end{array}$ & $\begin{array}{c}3.88 \mathrm{e} \\
-4\end{array}$ & $\begin{array}{l}3.85 e \\
-4\end{array}$ & $\begin{array}{l}2.16 e \\
-4\end{array}$ & $\begin{array}{l}1.60 e \\
-4\end{array}$ & $\begin{array}{c}5.20 e \\
-5\end{array}$ & $\begin{array}{c}3.77 e \\
-5\end{array}$ & $\begin{array}{c}3.47 e \\
-5\end{array}$ & $\begin{array}{c}2.06 e \\
-4\end{array}$ & $\begin{array}{c}3.30 e \\
-4\end{array}$ & $\begin{array}{l}1.27 e \\
-4\end{array}$ & $\begin{array}{c}2.39 e \\
-4\end{array}$ \\
\hline $\begin{array}{l}B 11_{t} \\
h e\end{array}$ & $\begin{array}{l}7.04 \mathrm{e} \\
-4\end{array}$ & $\begin{array}{c}9.10 \mathrm{e} \\
-4\end{array}$ & $\begin{array}{l}9.17 e \\
-4\end{array}$ & $\begin{array}{l}4.42 e \\
-4\end{array}$ & $\begin{array}{c}3.29 e \\
-4\end{array}$ & $\begin{array}{l}5.44 \mathrm{e} \\
-5\end{array}$ & $\begin{array}{c}3.95 e \\
-5\end{array}$ & $\begin{array}{c}3.64 \mathrm{e} \\
-4\end{array}$ & $\begin{array}{l}3.17 e \\
-4\end{array}$ & $\begin{array}{l}3.50 \mathrm{e} \\
-4\end{array}$ & $\begin{array}{l}1.22 \mathrm{e} \\
-4\end{array}$ & $\begin{array}{c}3.34 \mathrm{e} \\
-4\end{array}$ \\
\hline $\begin{array}{l}B 12_{a} \\
o t\end{array}$ & $\begin{array}{c}4.20 \mathrm{e} \\
-4\end{array}$ & $\begin{array}{c}4.34 \mathrm{e} \\
-4\end{array}$ & $\begin{array}{l}4.34 \mathrm{e} \\
-4\end{array}$ & $\begin{array}{l}3.24 \mathrm{e} \\
-4\end{array}$ & $\begin{array}{c}2.76 e \\
-4\end{array}$ & $\begin{array}{c}5.29 e \\
-5\end{array}$ & $\begin{array}{c}3.83 e \\
-5\end{array}$ & $\begin{array}{c}3.53 e \\
-4\end{array}$ & $\begin{array}{l}3.21 \mathrm{e} \\
-4\end{array}$ & $\begin{array}{c}3.26 e \\
-4\end{array}$ & $\begin{array}{c}2.91 e \\
-4\end{array}$ & $\begin{array}{c}4.80 \mathrm{e} \\
-4\end{array}$ \\
\hline $\begin{array}{l}B 12_{t} \\
h e\end{array}$ & $\begin{array}{l}0.001 \\
3\end{array}$ & $\begin{array}{l}0.002 \\
0\end{array}$ & $\begin{array}{l}0.002 \\
0\end{array}$ & $\begin{array}{l}7.91 e \\
-4\end{array}$ & $\begin{array}{c}3.85 e \\
-4\end{array}$ & $\begin{array}{c}5.36 \mathrm{e} \\
-5\end{array}$ & $\begin{array}{c}3.88 \mathrm{e} \\
-5\end{array}$ & $\begin{array}{c}3.58 \mathrm{e} \\
-5\end{array}$ & $\begin{array}{c}8.88 \mathrm{e} \\
-4\end{array}$ & $\begin{array}{c}9.13 e \\
-4\end{array}$ & $\begin{array}{l}0.001 \\
0\end{array}$ & $\begin{array}{l}0.002 \\
2\end{array}$ \\
\hline $\begin{array}{l}R 13 \\
\text { act }\end{array}$ & $\begin{array}{l}0.001 \\
5\end{array}$ & $\begin{array}{l}0.001 \\
5\end{array}$ & $\begin{array}{l}0.001 \\
6\end{array}$ & $\begin{array}{l}0.001 \\
3\end{array}$ & $\begin{array}{l}0.002 \\
1\end{array}$ & $\begin{array}{c}4.79 e \\
-5\end{array}$ & $\begin{array}{c}3.49 \mathrm{e} \\
-5\end{array}$ & $\begin{array}{c}3.22 \mathrm{e} \\
-5\end{array}$ & $\begin{array}{l}0.001 \\
1\end{array}$ & $\begin{array}{l}0.001 \\
2\end{array}$ & $\begin{array}{c}8.62 e \\
-4\end{array}$ & $\begin{array}{l}0.001 \\
6\end{array}$ \\
\hline $\begin{array}{l}R 13_{t} \\
\text { he }\end{array}$ & $\begin{array}{c}8.99 e \\
-4\end{array}$ & $\begin{array}{l}0.001 \\
2\end{array}$ & $\begin{array}{l}0.001 \\
2\end{array}$ & $\begin{array}{l}4.69 e \\
-4\end{array}$ & $\begin{array}{c}3.65 e \\
-4\end{array}$ & $\begin{array}{c}5.18 e \\
-5\end{array}$ & $\begin{array}{c}3.77 e \\
-5\end{array}$ & $\begin{array}{c}3.47 \mathrm{e} \\
-5\end{array}$ & $\begin{array}{l}6.27 e \\
-4\end{array}$ & $\begin{array}{l}6.38 \mathrm{e} \\
-4\end{array}$ & $\begin{array}{c}5.29 e \\
-4\end{array}$ & $\begin{array}{c}9.75 \mathrm{e} \\
-4\end{array}$ \\
\hline $\begin{array}{l}R 10 \\
a c t_{1}\end{array}$ & $\begin{array}{l}0.001 \\
8\end{array}$ & $\begin{array}{l}0.002 \\
5\end{array}$ & $\begin{array}{l}0.002 \\
5\end{array}$ & $\begin{array}{l}0.001 \\
3\end{array}$ & $\begin{array}{l}0.001 \\
5\end{array}$ & $\begin{array}{l}4.87 e \\
-5\end{array}$ & $\begin{array}{l}3.54 \mathrm{e} \\
-5\end{array}$ & $\begin{array}{c}3.26 e \\
-5\end{array}$ & $\begin{array}{l}0.001 \\
2\end{array}$ & $\begin{array}{l}0.002 \\
5\end{array}$ & $\begin{array}{l}0.001 \\
9\end{array}$ & $\begin{array}{l}0.003 \\
8\end{array}$ \\
\hline $\begin{array}{l}R 10 t \\
h e_{1}\end{array}$ & $\begin{array}{l}0.001 \\
4\end{array}$ & $\begin{array}{l}0.002 \\
3\end{array}$ & $\begin{array}{l}0.002 \\
2\end{array}$ & $\begin{array}{l}7.25 e \\
-4\end{array}$ & $\begin{array}{c}4.59 \mathrm{e} \\
-4\end{array}$ & $\begin{array}{c}5.04 e \\
-5\end{array}$ & $\begin{array}{c}3.65 e \\
-5\end{array}$ & $\begin{array}{c}3.37 e \\
-5\end{array}$ & $\begin{array}{c}3.01 \mathrm{e} \\
-4\end{array}$ & $\begin{array}{l}0.003 \\
5\end{array}$ & $\begin{array}{l}0.002 \\
9\end{array}$ & $\begin{array}{l}0.005 \\
2\end{array}$ \\
\hline $\begin{array}{l}R 10 \\
\text { act }_{2}\end{array}$ & $\begin{array}{l}0.001 \\
4\end{array}$ & $\begin{array}{l}0.001 \\
9\end{array}$ & $\begin{array}{l}0.001 \\
9\end{array}$ & $\begin{array}{l}9.49 \mathrm{e} \\
-4\end{array}$ & $\begin{array}{l}0.001 \\
1\end{array}$ & $\begin{array}{l}4.97 e \\
-5\end{array}$ & $\begin{array}{l}3.61 \mathrm{e} \\
-5\end{array}$ & $\begin{array}{c}3.33 e \\
-5\end{array}$ & $\begin{array}{l}6.94 \mathrm{e} \\
-4\end{array}$ & $\begin{array}{l}7.17 e \\
-4\end{array}$ & $\begin{array}{l}5.15 e \\
-4\end{array}$ & $\begin{array}{l}0.001 \\
1\end{array}$ \\
\hline $\begin{array}{l}R 10 t \\
h e_{2}\end{array}$ & $\begin{array}{c}4.46 e \\
-4\end{array}$ & $\begin{array}{l}8.66 e \\
-4\end{array}$ & $\begin{array}{l}7.35 e \\
-4\end{array}$ & $\begin{array}{l}2.88 \mathrm{e} \\
-4\end{array}$ & $\begin{array}{l}1.35 e \\
-4\end{array}$ & $\begin{array}{l}5.03 e \\
-5\end{array}$ & $\begin{array}{c}3.65 e \\
-5\end{array}$ & $\begin{array}{c}3.36 e \\
-5\end{array}$ & $\begin{array}{l}3.41 e \\
-4\end{array}$ & $\begin{array}{c}3.51 e \\
-4\end{array}$ & $\begin{array}{c}2.85 e \\
-4\end{array}$ & $\begin{array}{c}5.06 \mathrm{e} \\
-4\end{array}$ \\
\hline $116_{a c t}$ & $\begin{array}{c}8.02 e \\
-4\end{array}$ & $\begin{array}{l}0.001 \\
1\end{array}$ & $\begin{array}{l}0.001 \\
1\end{array}$ & $\begin{array}{l}5.78 \mathrm{e} \\
-4\end{array}$ & $\begin{array}{c}3.80 \mathrm{e} \\
-4\end{array}$ & $\begin{array}{c}4.78 e \\
-5\end{array}$ & $\begin{array}{c}3.46 e \\
-5\end{array}$ & $\begin{array}{c}3.19 e \\
-5\end{array}$ & $\begin{array}{c}4.51 e \\
-4\end{array}$ & $\begin{array}{c}4.68 \mathrm{e} \\
-4\end{array}$ & $\begin{array}{c}4.45 e \\
-4\end{array}$ & $\begin{array}{c}7.89 \mathrm{e} \\
-4\end{array}$ \\
\hline $\begin{array}{l}116_{t h} \\
e\end{array}$ & $\begin{array}{l}0.002 \\
0\end{array}$ & $\begin{array}{l}0.003 \\
1\end{array}$ & $\begin{array}{l}0.003 \\
0\end{array}$ & $\begin{array}{l}9.00 \mathrm{e} \\
-4\end{array}$ & $\begin{array}{c}8.05 e \\
-4\end{array}$ & $\begin{array}{l}5.09 e \\
-5\end{array}$ & $\begin{array}{c}3.69 \mathrm{e} \\
-5\end{array}$ & $\begin{array}{c}3.40 \mathrm{e} \\
-5\end{array}$ & $\begin{array}{l}0.001 \\
2\end{array}$ & $\begin{array}{l}0.001 \\
3\end{array}$ & $\begin{array}{l}0.001 \\
1\end{array}$ & $\begin{array}{l}0.002 \\
0\end{array}$ \\
\hline $\begin{array}{l}C 14 \\
a c t_{1}\end{array}$ & $\begin{array}{l}8.50 \mathrm{e} \\
-4\end{array}$ & $\begin{array}{l}9.24 \mathrm{e} \\
-4\end{array}$ & $\begin{array}{l}9.24 \mathrm{e} \\
-4\end{array}$ & $\begin{array}{l}4.67 e \\
-4\end{array}$ & $\begin{array}{l}4.07 e \\
-4\end{array}$ & $\begin{array}{l}4.91 \mathrm{e} \\
-5\end{array}$ & $\begin{array}{c}3.55 e \\
-5\end{array}$ & $\begin{array}{c}3.28 \mathrm{e} \\
-5\end{array}$ & $\begin{array}{l}6.34 \mathrm{e} \\
-4\end{array}$ & $\begin{array}{l}6.42 \mathrm{e} \\
-4\end{array}$ & $\begin{array}{l}5.09 \mathrm{e} \\
-4\end{array}$ & $\begin{array}{c}9.67 \mathrm{e} \\
-4\end{array}$ \\
\hline $\begin{array}{l}C 14 t \\
h e_{1}\end{array}$ & $\begin{array}{c}8.29 e \\
-4\end{array}$ & $\begin{array}{l}0.001 \\
2\end{array}$ & $\begin{array}{l}0.001 \\
2\end{array}$ & $\begin{array}{l}7.24 \mathrm{e} \\
-4\end{array}$ & $\begin{array}{c}3.90 \mathrm{e} \\
-4\end{array}$ & $\begin{array}{c}4.96 e \\
-5\end{array}$ & $\begin{array}{c}3.60 \mathrm{e} \\
-5\end{array}$ & $\begin{array}{c}3.32 \mathrm{e} \\
-5\end{array}$ & $\begin{array}{c}5.23 e \\
-4\end{array}$ & $\begin{array}{c}5.29 e \\
-4\end{array}$ & $\begin{array}{c}4.79 e \\
-4\end{array}$ & $\begin{array}{c}8.31 \mathrm{e} \\
-4\end{array}$ \\
\hline
\end{tabular}




\begin{tabular}{|c|c|c|c|c|c|c|c|c|c|c|c|c|}
\hline & $V_{t m}$ & $\begin{array}{l}B P V_{t} \\
m\end{array}$ & $\begin{array}{l}R V_{t m a} \\
\left.a d f^{\prime}\right)\end{array}$ & $\begin{array}{l}R V_{t m a} \\
\left.a d f^{2}\right)\end{array}$ & $\begin{array}{l}\text { MRtP } \\
\text { ar }\end{array}$ & $\begin{array}{l}M R_{t G} \\
\kappa\end{array}$ & $\begin{array}{l}M R t R \\
s\end{array}$ & $Z$ & $\begin{array}{l}\text { RRtP } \\
\text { ar }\end{array}$ & $\begin{array}{l}R R t G \\
\kappa\end{array}$ & $s$ & $\begin{array}{l}R R t \\
Z\end{array}$ \\
\hline 14 & -4 & 1 & 2 & -4 & -4 & -5 & 5 & 5 & 1 & 1 & 1 & 5 \\
\hline & -4 & 2 & 1 & -4 & -4 & -5 & -5 & -5 & -4 & -4 & -4 & $\begin{array}{c}9.76 \\
-4\end{array}$ \\
\hline act & 4 & 8 & 8 & -4 & -4 & -5 & -5 & 5 & $\begin{array}{l}0.010 \\
2\end{array}$ & 8 & $\begin{array}{c}6.52 \\
-4\end{array}$ & $\begin{array}{l}0.0 \\
7\end{array}$ \\
\hline he & $\begin{array}{l}0.025 \\
8\end{array}$ & $\begin{array}{l}0.046 \\
8\end{array}$ & $\begin{array}{l}0.051 \\
1\end{array}$ & $\begin{array}{l}0.020 \\
8\end{array}$ & $\begin{array}{l}0.005 \\
1\end{array}$ & $\begin{array}{c}4.96 e \\
-5\end{array}$ & $\begin{array}{c}3.64 e \\
-5\end{array}$ & $\begin{array}{c}3.36 € \\
-5\end{array}$ & $\begin{array}{l}0.0 \\
5\end{array}$ & $\begin{array}{l}0.019 \\
1\end{array}$ & $\begin{array}{c}4.64 \mathrm{e} \\
-4\end{array}$ & 0.02 \\
\hline
\end{tabular}

Notes. Table 6 reports the average magnitude of risk (mean volatility) $(\overline{\boldsymbol{R}})$ for both actual and theoretical BRIC Eurobond prices. Risk (volatility) estimates are split into three groups: realized volatility, monthly range, and realized range estimates. All average values reported, are t-test significant in a $5 \%$ significance level.

Table 7.

Average Sharpe ratio $\overline{S R}$ ).

\begin{tabular}{|c|c|c|c|c|c|c|c|c|c|c|c|c|}
\hline & $R V_{t m}$ & $\begin{array}{l}B P V_{t} \\
m\end{array}$ & $\begin{array}{l}R V_{t m a} \\
\left.a d^{\prime \prime}\right)\end{array}$ & $\begin{array}{l}R V_{t m a} \\
\left.a d^{2}\right)\end{array}$ & $\begin{array}{l}M R_{t} P \\
a r\end{array}$ & $\begin{array}{l}M R_{t} \\
G K\end{array}$ & $\begin{array}{l}M R_{t} \\
R S\end{array}$ & $\begin{array}{l}M R_{t} \\
Y Z\end{array}$ & $\begin{array}{l}R R t P \\
a t\end{array}$ & $\begin{array}{l}R R t G \\
\kappa\end{array}$ & $\begin{array}{l}R R_{t} R \\
S\end{array}$ & $\begin{array}{l}R R_{t} Y \\
Z\end{array}$ \\
\hline $\begin{array}{l}B 14_{a} \\
c t\end{array}$ & $\begin{array}{l}-0.3 \\
401\end{array}$ & $\begin{array}{l}-0.3 \\
117\end{array}$ & $\begin{array}{l}-0.31 \\
19\end{array}$ & $\begin{array}{l}-0.47 \\
47\end{array}$ & $\begin{array}{l}-0.4 \\
467\end{array}$ & $\begin{array}{l}-5.3 \\
9\end{array}$ & $\begin{array}{l}-7.4 \\
4\end{array}$ & $\begin{array}{l}-8.0 \\
8\end{array}$ & $\begin{array}{l}-0.5 \\
314\end{array}$ & $\begin{array}{l}-0.5 \\
206\end{array}$ & $\begin{array}{l}-0.5 \\
879\end{array}$ & $\begin{array}{l}-0.3 \\
566\end{array}$ \\
\hline $\begin{array}{l}B 14_{\text {th }} \\
e\end{array}$ & $\begin{array}{l}-0.2 \\
557\end{array}$ & $\begin{array}{l}-0.1 \\
600\end{array}$ & $\begin{array}{l}-0.16 \\
79\end{array}$ & $\begin{array}{l}-0.43 \\
78\end{array}$ & $\begin{array}{l}-0.5 \\
902\end{array}$ & $\begin{array}{l}-5.8 \\
5\end{array}$ & $\begin{array}{l}-8.0 \\
5\end{array}$ & $\begin{array}{l}-8.7 \\
4\end{array}$ & $\begin{array}{l}-0.3 \\
962\end{array}$ & $\begin{array}{l}-0.3 \\
850\end{array}$ & $\begin{array}{l}-0.4 \\
405\end{array}$ & $\begin{array}{l}-0.2 \\
446\end{array}$ \\
\hline $\begin{array}{l}B 11_{a} \\
c t\end{array}$ & $\begin{array}{l}-2.0 \\
4\end{array}$ & $\begin{array}{l}-1.6 \\
1\end{array}$ & -1.62 & -2.89 & $\begin{array}{l}-3.9 \\
1\end{array}$ & $\begin{array}{l}-12 . \\
02\end{array}$ & $\begin{array}{l}-16 . \\
59\end{array}$ & $\begin{array}{l}-18 . \\
00\end{array}$ & $\begin{array}{l}-3.0 \\
4\end{array}$ & 1.90 & $\begin{array}{l}-4.9 \\
3\end{array}$ & $\begin{array}{l}-2.6 \\
2\end{array}$ \\
\hline $\begin{array}{l}B 11_{t h} \\
e\end{array}$ & $\begin{array}{l}-1.0 \\
4\end{array}$ & $\begin{array}{l}-0.8 \\
045\end{array}$ & $\begin{array}{l}-0.79 \\
85\end{array}$ & -1.66 & $\begin{array}{l}-2.2 \\
3\end{array}$ & $\begin{array}{l}-13 \\
46\end{array}$ & $\begin{array}{l}-18 \\
54\end{array}$ & $\begin{array}{l}-20 \\
11\end{array}$ & $\begin{array}{l}-2.3 \\
1\end{array}$ & 2.09 & $\begin{array}{l}-6.0 \\
1\end{array}$ & $\begin{array}{l}-2.1 \\
9\end{array}$ \\
\hline $\begin{array}{l}B 12_{a} \\
{ }_{c t}\end{array}$ & $\begin{array}{l}-3.4 \\
7\end{array}$ & $\begin{array}{l}-3.3 \\
5\end{array}$ & -3.35 & -4.49 & $\begin{array}{l}-5.2 \\
8\end{array}$ & $\begin{array}{l}-27 \\
52\end{array}$ & $\begin{array}{l}-38 \\
00\end{array}$ & $\begin{array}{l}-41 \\
23\end{array}$ & $\begin{array}{l}-4.5 \\
3\end{array}$ & $\begin{array}{l}-4.4 \\
7\end{array}$ & $\begin{array}{l}-5.0 \\
0\end{array}$ & $\begin{array}{l}-3.0 \\
4\end{array}$ \\
\hline $\begin{array}{l}B 12_{t h} \\
e\end{array}$ & $\begin{array}{l}-1.5 \\
2\end{array}$ & $\begin{array}{l}-1.0 \\
1\end{array}$ & $\begin{array}{l}-0.96 \\
25\end{array}$ & -2.49 & $\begin{array}{l}-5.1 \\
1\end{array}$ & $\begin{array}{l}-36 \\
68\end{array}$ & $\begin{array}{l}-50 . \\
59\end{array}$ & $\begin{array}{l}-54 \\
88\end{array}$ & $\begin{array}{l}-2.2 \\
1\end{array}$ & $\begin{array}{l}-2.1 \\
5\end{array}$ & $\begin{array}{l}-1.9 \\
6\end{array}$ & $\begin{array}{l}-0.9 \\
048\end{array}$ \\
\hline $\begin{array}{l}R 13_{a} \\
a t\end{array}$ & $\begin{array}{l}0.170 \\
9\end{array}$ & $\begin{array}{l}0.172 \\
2\end{array}$ & 0.1695 & 0.2060 & $\begin{array}{l}0.130 \\
0\end{array}$ & 5.52 & 7.59 & 8.24 & $\begin{array}{l}0.232 \\
7\end{array}$ & $\begin{array}{l}0.227 \\
6\end{array}$ & $\begin{array}{l}0.307 \\
1\end{array}$ & $\begin{array}{l}0.161 \\
2\end{array}$ \\
\hline $\begin{array}{l}R 13_{t} \\
\text { he }\end{array}$ & $\begin{array}{l}0.771 \\
8\end{array}$ & $\begin{array}{l}0.571 \\
3\end{array}$ & 0.5736 & 1.48 & 1.90 & $\begin{array}{l}13.3 \\
9\end{array}$ & $\begin{array}{l}18.4 \\
3\end{array}$ & $\begin{array}{l}20.0 \\
0\end{array}$ & 1.11 & 1.09 & 1.31 & $\begin{array}{l}0.712 \\
2\end{array}$ \\
\hline $\begin{array}{l}R 10 \\
a c t_{1}\end{array}$ & $\begin{array}{l}-0.1 \\
595\end{array}$ & $\begin{array}{l}-0.1 \\
197\end{array}$ & $\begin{array}{l}-0.11 \\
82\end{array}$ & $\begin{array}{l}-0.22 \\
35\end{array}$ & $\begin{array}{l}-0.1 \\
977\end{array}$ & $\begin{array}{l}-6.0 \\
4\end{array}$ & $\begin{array}{l}-8.3 \\
2\end{array}$ & $\begin{array}{l}-9.0 \\
2\end{array}$ & $\begin{array}{l}-0.2 \\
527\end{array}$ & $\begin{array}{l}0.116 \\
0\end{array}$ & $\begin{array}{l}0.151 \\
6\end{array}$ & $\begin{array}{l}0.078 \\
3\end{array}$ \\
\hline $\begin{array}{l}R 10 t \\
h e_{1}\end{array}$ & $\begin{array}{l}-1.3 \\
3\end{array}$ & $\begin{array}{l}-0.8 \\
068\end{array}$ & $\begin{array}{l}-0.84 \\
56\end{array}$ & -2.58 & $\begin{array}{l}-4.0 \\
6\end{array}$ & $\begin{array}{l}-37 \\
04\end{array}$ & $\begin{array}{l}-51 . \\
09\end{array}$ & $\begin{array}{l}-55 \\
43\end{array}$ & $\begin{array}{l}-6.2 \\
0\end{array}$ & $\begin{array}{l}0.539 \\
4\end{array}$ & $\begin{array}{l}0.652 \\
9\end{array}$ & $\begin{array}{l}0.360 \\
5\end{array}$ \\
\hline $\begin{array}{l}R 10 \\
\text { act }_{2}\end{array}$ & $\begin{array}{l}0.081 \\
1\end{array}$ & $\begin{array}{l}0.061 \\
4\end{array}$ & 0.0619 & 0.1218 & $\begin{array}{l}0.104 \\
7\end{array}$ & 2.32 & 3.20 & 3.47 & $\begin{array}{l}0.166 \\
3\end{array}$ & $\begin{array}{l}0.161 \\
1\end{array}$ & $\begin{array}{l}0.224 \\
2\end{array}$ & $\begin{array}{l}0.110 \\
6\end{array}$ \\
\hline $\begin{array}{l}R 10 t \\
h e_{2}\end{array}$ & $\begin{array}{l}-0.9 \\
205\end{array}$ & $\begin{array}{l}-0.4 \\
741\end{array}$ & $\begin{array}{l}-0.55 \\
87\end{array}$ & -1.42 & $\begin{array}{l}-3.0 \\
5\end{array}$ & $\begin{array}{l}-8.1 \\
7\end{array}$ & $\begin{array}{l}-11 \\
26\end{array}$ & $\begin{array}{l}-12 \\
22\end{array}$ & $\begin{array}{l}-1.2 \\
1\end{array}$ & $\begin{array}{l}-1.1 \\
7\end{array}$ & $\begin{array}{l}-1.4 \\
4\end{array}$ & $\begin{array}{l}-0.8 \\
123\end{array}$ \\
\hline $116_{a c t}$ & 1.74 & 1.24 & 1.24 & 2.41 & 3.67 & $\begin{array}{l}29.1 \\
7\end{array}$ & $\begin{array}{l}40.2 \\
9\end{array}$ & $\begin{array}{l}43.7 \\
1\end{array}$ & 3.09 & 2.98 & 3.13 & 1.77 \\
\hline $116_{\text {the }}$ & $\begin{array}{l}0.927 \\
7\end{array}$ & $\begin{array}{l}0.586 \\
1\end{array}$ & 0.6108 & 2.02 & 2.25 & $\begin{array}{l}35.6 \\
6\end{array}$ & $\begin{array}{l}49.1 \\
6\end{array}$ & $\begin{array}{l}53.3 \\
3\end{array}$ & 1.49 & 1.43 & 1.67 & $\begin{array}{l}0.906 \\
3\end{array}$ \\
\hline
\end{tabular}




\begin{tabular}{|c|c|c|c|c|c|c|c|c|c|c|c|c|}
\hline & $R V_{t m}$ & $\begin{array}{l}B P V_{t} \\
m\end{array}$ & $\begin{array}{l}R V_{t m a} \\
\left.a d^{\prime \prime}\right)\end{array}$ & $\begin{array}{l}R V_{t m a} \\
\left.a d f^{2}\right)\end{array}$ & $\begin{array}{l}M R_{t} P \\
a r\end{array}$ & $\begin{array}{l}M R t \\
G K\end{array}$ & $\begin{array}{l}M R_{t} \\
R S\end{array}$ & $\begin{array}{l}M R_{t} \\
Y Z\end{array}$ & $\begin{array}{l}R_{a t} \\
R_{t P}\end{array}$ & $\begin{array}{l}R R_{t G} \\
\kappa\end{array}$ & $\begin{array}{l}R R_{t R} \\
S\end{array}$ & $\begin{array}{l}R R_{t Y} \\
Z\end{array}$ \\
\hline $\begin{array}{l}C 14 \\
a c t_{1}\end{array}$ & 2.10 & 1.93 & 1.93 & 3.82 & 4.38 & $\begin{array}{l}36.3 \\
4\end{array}$ & $\begin{array}{l}50.1 \\
8\end{array}$ & $\begin{array}{l}54.4 \\
4\end{array}$ & 2.81 & 2.78 & 3.50 & 1.85 \\
\hline $\begin{array}{l}C 14 t \\
h e_{1}\end{array}$ & 2.79 & 1.90 & 1.89 & 3.19 & 5.93 & $\begin{array}{l}46.5 \\
9\end{array}$ & $\begin{array}{l}64.2 \\
7\end{array}$ & $\begin{array}{l}69.7 \\
3\end{array}$ & 4.42 & 4.37 & 4.82 & 2.78 \\
\hline $\begin{array}{l}C 14 \\
\text { act }_{2}\end{array}$ & 2.20 & 1.94 & 1.79 & 3.12 & 3.70 & $\begin{array}{l}44.2 \\
3\end{array}$ & $\begin{array}{l}61.0 \\
5\end{array}$ & $\begin{array}{l}66.2 \\
3\end{array}$ & 2.28 & 2.22 & 2.73 & 1.44 \\
\hline $\begin{array}{l}C 14 \\
\text { act }_{2}\end{array}$ & 2.72 & 1.70 & 1.77 & 3.28 & 5.82 & $\begin{array}{l}40.7 \\
3\end{array}$ & $\begin{array}{l}56.1 \\
6\end{array}$ & $\begin{array}{l}60.9 \\
3\end{array}$ & 3.19 & 3.15 & 3.58 & 2.09 \\
\hline $\begin{array}{l}C 15_{a} \\
c t\end{array}$ & 2.04 & 1.62 & 1.63 & 6.90 & 4.39 & $\begin{array}{l}58.6 \\
8\end{array}$ & $\begin{array}{l}81.1 \\
8\end{array}$ & $\begin{array}{l}88.0 \\
7\end{array}$ & $\begin{array}{l}0.151 \\
7\end{array}$ & $\begin{array}{l}0.147 \\
2\end{array}$ & 4.46 & $\begin{array}{l}0.105 \\
1\end{array}$ \\
\hline$C 15_{t}$ & $\begin{array}{l}0.084 \\
8\end{array}$ & $\begin{array}{l}0.046 \\
8\end{array}$ & 0.0429 & 0.1052 & $\begin{array}{l}0.433 \\
7\end{array}$ & $\begin{array}{l}44.1 \\
8\end{array}$ & $\begin{array}{l}60.2 \\
2\end{array}$ & $\begin{array}{l}65.2 \\
9\end{array}$ & $\begin{array}{l}0.118 \\
6\end{array}$ & $\begin{array}{l}0.114 \\
8\end{array}$ & 47.19 & $\begin{array}{l}0.081 \\
7\end{array}$ \\
\hline
\end{tabular}

Notes. Table 7 reports the average Sharpe ratio $(\overline{S R})$ for both actual and theoretical BRIC Eurobond prices. Sharpe ratio estimates $\left(S R_{t}\right)$ are split into the three groups of risk estimators: realized volatility, monthly range, and realized range estimates. All average values reported, are t-test significant in a $5 \%$ significance level.

Table 8.

Mean magnitude of jumps $(\bar{M})$.

\begin{tabular}{|c|c|c|c|c|c|c|c|c|c|c|c|c|}
\hline & $R V_{t m}$ & $\begin{array}{l}B P V_{t} \\
m\end{array}$ & $\begin{array}{l}R V_{t m a} \\
\left.a d f^{n}\right)\end{array}$ & $\begin{array}{l}R V_{t m a} \\
a d / 2)\end{array}$ & $\begin{array}{l}M R_{t} P \\
a r\end{array}$ & $\underset{\kappa}{M R_{t} G}$ & $\begin{array}{l}M R_{t R} \\
S\end{array}$ & $\begin{array}{l}M R A Y \\
Z\end{array}$ & $\begin{array}{l}R_{t} R_{t} \\
a r\end{array}$ & $\begin{array}{l}R R_{t G} \\
\kappa\end{array}$ & $\begin{array}{l}R R_{t} R \\
S\end{array}$ & $\begin{array}{l}R R t Y \\
Z\end{array}$ \\
\hline $\begin{array}{l}B 14_{a} \\
c t\end{array}$ & $\begin{array}{c}5.53 e \\
-4\end{array}$ & $\begin{array}{c}6.09 e \\
-4\end{array}$ & $\begin{array}{l}7.60 \mathrm{e} \\
-4\end{array}$ & $\begin{array}{l}7.60 \mathrm{e} \\
-4\end{array}$ & $\begin{array}{c}1.59 \mathrm{e} \\
-4\end{array}$ & $\begin{array}{c}3.21 \mathrm{e} \\
-5\end{array}$ & $\begin{array}{c}2.18 e \\
-5\end{array}$ & $\begin{array}{c}2.05 e \\
-5\end{array}$ & $\begin{array}{c}1.26 \mathrm{e} \\
-4\end{array}$ & $\begin{array}{c}1.24 \mathrm{e} \\
-4\end{array}$ & $\begin{array}{c}5.62 e \\
-5\end{array}$ & $\begin{array}{c}5.78 \mathrm{e} \\
-4\end{array}$ \\
\hline $\begin{array}{l}B 14_{t} \\
h e\end{array}$ & $\begin{array}{l}0.001 \\
3\end{array}$ & $\begin{array}{l}6.67 e \\
-4\end{array}$ & $\begin{array}{l}0.002 \\
3\end{array}$ & $\begin{array}{l}0.002 \\
2\end{array}$ & $\begin{array}{c}5.32 e \\
-4\end{array}$ & $\begin{array}{l}1.71 e \\
-5\end{array}$ & $\begin{array}{l}1.83 e \\
-5\end{array}$ & $\begin{array}{l}1.70 e \\
-5\end{array}$ & $\begin{array}{l}0.001 \\
7\end{array}$ & $\begin{array}{l}0.002 \\
1\end{array}$ & $\begin{array}{l}0.001 \\
8\end{array}$ & $\begin{array}{l}0.002 \\
0\end{array}$ \\
\hline $\begin{array}{l}B 11_{a} \\
a t\end{array}$ & $\begin{array}{l}1.48 e \\
-4\end{array}$ & $\begin{array}{l}1.80 e \\
-4\end{array}$ & $\begin{array}{l}2.21 \mathrm{e} \\
-4\end{array}$ & $\begin{array}{l}2.18 e \\
-4\end{array}$ & $\begin{array}{c}4.41 e \\
-5\end{array}$ & $\begin{array}{c}3.31 e \\
-5\end{array}$ & $\begin{array}{c}2.41 e \\
-5\end{array}$ & $\begin{array}{c}2.11 e \\
-5\end{array}$ & $\begin{array}{c}6.51 e \\
-5\end{array}$ & $\begin{array}{l}6.87 e \\
-4\end{array}$ & $\begin{array}{c}3.02 e \\
-4\end{array}$ & $\begin{array}{c}2.59 e \\
-4\end{array}$ \\
\hline$B 11_{t}$ & $\begin{array}{l}0.001 \\
3\end{array}$ & $\begin{array}{l}1.23 e \\
-4\end{array}$ & $\begin{array}{l}5.90 e \\
-4\end{array}$ & $\begin{array}{l}5.97 e \\
-4\end{array}$ & $\begin{array}{c}3.68 \mathrm{e} \\
-4\end{array}$ & $\begin{array}{c}3.40 e \\
-5\end{array}$ & $\begin{array}{c}1.95 e \\
-5\end{array}$ & $\begin{array}{c}2.08 \mathrm{e} \\
-5\end{array}$ & $\begin{array}{c}6.23 e \\
-4\end{array}$ & $\begin{array}{l}6.23 e \\
-4\end{array}$ & $\begin{array}{c}6.23 e \\
-4\end{array}$ & $\begin{array}{c}6.23 e \\
-4\end{array}$ \\
\hline $\begin{array}{l}B 12_{a} \\
c t\end{array}$ & $\begin{array}{c}2.07 e \\
-4\end{array}$ & $\begin{array}{c}2.86 e \\
-4\end{array}$ & $\begin{array}{l}1.93 e \\
-4\end{array}$ & $\begin{array}{l}1.88 \mathrm{e} \\
-4\end{array}$ & $\begin{array}{c}6.62 e \\
-4\end{array}$ & $\begin{array}{c}2.60 \mathrm{e} \\
-5\end{array}$ & $\begin{array}{c}1.83 e \\
-5\end{array}$ & $\begin{array}{l}1.99 e \\
-5\end{array}$ & $\begin{array}{c}7.60 e \\
-5\end{array}$ & $\begin{array}{c}8.72 \mathrm{e} \\
-5\end{array}$ & $\begin{array}{c}7.27 e \\
-5\end{array}$ & $\begin{array}{c}1.33 e \\
-4\end{array}$ \\
\hline $\begin{array}{l}B 12_{t} \\
{ }_{h e}\end{array}$ & $\begin{array}{l}0.001 \\
6\end{array}$ & $\begin{array}{c}8.56 e \\
-4\end{array}$ & $\begin{array}{l}0.002 \\
1\end{array}$ & $\begin{array}{l}0.002 \\
3\end{array}$ & $\begin{array}{c}3.56 \mathrm{e} \\
-4\end{array}$ & $\begin{array}{c}3.30 e \\
-5\end{array}$ & $\begin{array}{c}2.25 e \\
-5\end{array}$ & $\begin{array}{c}2.21 \mathrm{e} \\
-5\end{array}$ & $\begin{array}{l}6.14 e \\
-4\end{array}$ & $\begin{array}{l}6.14 e \\
-4\end{array}$ & $\begin{array}{l}6.14 \mathrm{e} \\
-4\end{array}$ & $\begin{array}{l}6.14 \mathrm{e} \\
-4\end{array}$ \\
\hline $\begin{array}{l}R 13 \\
a c t\end{array}$ & $\begin{array}{c}3.81 e \\
-4\end{array}$ & $\begin{array}{l}0.001 \\
3\end{array}$ & $\begin{array}{l}7.87 e \\
-4\end{array}$ & $\begin{array}{l}7.84 \mathrm{e} \\
-4\end{array}$ & $\begin{array}{l}0.001 \\
5\end{array}$ & $\begin{array}{c}2.13 e \\
-5\end{array}$ & $\begin{array}{l}1.42 e \\
-5\end{array}$ & $\begin{array}{l}1.14 \mathrm{e} \\
-5\end{array}$ & $\begin{array}{c}2.07 e \\
-4\end{array}$ & $\begin{array}{c}2.88 \mathrm{e} \\
-4\end{array}$ & $\begin{array}{c}1.26 e \\
-4\end{array}$ & $\begin{array}{c}3.74 \mathrm{e} \\
-4\end{array}$ \\
\hline $\begin{array}{l}R 13_{t} \\
h e\end{array}$ & $\begin{array}{l}0.001 \\
6\end{array}$ & $\begin{array}{c}4.03 e \\
-4\end{array}$ & $\begin{array}{l}0.001 \\
2\end{array}$ & $\begin{array}{l}0.001 \\
1\end{array}$ & $\begin{array}{c}8.35 e \\
-4\end{array}$ & $\begin{array}{c}9.54 \mathrm{e} \\
-6\end{array}$ & $\begin{array}{c}9.60 \mathrm{e} \\
-6\end{array}$ & $\begin{array}{c}9.18 e \\
-6\end{array}$ & $\begin{array}{l}0.001 \\
9\end{array}$ & $\begin{array}{l}0.002 \\
0\end{array}$ & $\begin{array}{l}0.001 \\
5\end{array}$ & $\begin{array}{c}7.89 e \\
-4\end{array}$ \\
\hline $\begin{array}{l}R 10 \\
a c t_{1}\end{array}$ & $\begin{array}{c}3.71 e \\
-4\end{array}$ & $\begin{array}{l}0.001 \\
3\end{array}$ & $\begin{array}{l}0.002 \\
1\end{array}$ & $\begin{array}{l}0.002 \\
1\end{array}$ & $\begin{array}{c}7.86 e \\
-4\end{array}$ & $\begin{array}{c}3.20 \mathrm{e} \\
-5\end{array}$ & $\begin{array}{c}2.21 e \\
-5\end{array}$ & $\begin{array}{c}2.09 e \\
-5\end{array}$ & $\begin{array}{l}0.002 \\
8\end{array}$ & $\begin{array}{l}0.003 \\
6\end{array}$ & $\begin{array}{l}0.002 \\
9\end{array}$ & $\begin{array}{l}0.005 \\
2\end{array}$ \\
\hline $\begin{array}{l}R 10 t \\
h e_{i}\end{array}$ & $\begin{array}{l}6.42 \mathrm{e} \\
-4\end{array}$ & $\begin{array}{c}2.72 \mathrm{e} \\
-4\end{array}$ & $\begin{array}{l}7.74 \mathrm{e} \\
-4\end{array}$ & $\begin{array}{l}6.48 \mathrm{e} \\
-4\end{array}$ & $\begin{array}{l}1.88 \mathrm{e} \\
-4\end{array}$ & $\begin{array}{c}2.51 \mathrm{e} \\
-5\end{array}$ & $\begin{array}{l}1.57 e \\
-5\end{array}$ & $\begin{array}{l}1.45 e \\
-5\end{array}$ & $\begin{array}{c}3.03 e \\
-4\end{array}$ & $\begin{array}{l}0.003 \\
2\end{array}$ & $\begin{array}{l}0.002 \\
8\end{array}$ & $\begin{array}{l}0.004 \\
9\end{array}$ \\
\hline $\begin{array}{l}R 10 \\
\text { act }_{2}\end{array}$ & $\begin{array}{l}6.10 e \\
-4\end{array}$ & $\begin{array}{c}6.52 e \\
-4\end{array}$ & $\begin{array}{l}7.95 e \\
-4\end{array}$ & $\begin{array}{l}9.66 \mathrm{e} \\
-4\end{array}$ & $\begin{array}{c}3.92 \mathrm{e} \\
-4\end{array}$ & $\begin{array}{c}3.02 e \\
-5\end{array}$ & $\begin{array}{c}1.83 e \\
-5\end{array}$ & $\begin{array}{l}1.55 e \\
-5\end{array}$ & $\begin{array}{l}1.69 e \\
-4\end{array}$ & $\begin{array}{l}1.73 e \\
-4\end{array}$ & $\begin{array}{l}6.50 \mathrm{e} \\
-5\end{array}$ & $\begin{array}{c}3.46 \mathrm{e} \\
-4\end{array}$ \\
\hline $\begin{array}{l}R 10 t \\
h e_{2}\end{array}$ & $\begin{array}{c}5.57 e \\
-4\end{array}$ & $\begin{array}{c}2.42 \mathrm{e} \\
-4\end{array}$ & $\begin{array}{l}8.41 e \\
-4\end{array}$ & $\begin{array}{l}6.27 e \\
-4\end{array}$ & $\begin{array}{c}1.81 \mathrm{e} \\
-4\end{array}$ & $\begin{array}{c}2.59 e \\
-5\end{array}$ & $\begin{array}{l}1.44 \mathrm{e} \\
-5\end{array}$ & $\begin{array}{c}1.34 \mathrm{e} \\
-5\end{array}$ & $\begin{array}{c}2.20 \mathrm{e} \\
-4\end{array}$ & $\begin{array}{c}2.31 \mathrm{e} \\
-4\end{array}$ & $\begin{array}{c}1.99 e \\
-4\end{array}$ & $\begin{array}{c}2.98 \mathrm{e} \\
-4\end{array}$ \\
\hline
\end{tabular}




\begin{tabular}{|c|c|c|c|c|c|c|c|c|c|c|c|c|}
\hline & $R V_{t m}$ & $\begin{array}{l}B P V_{t} \\
m\end{array}$ & $\begin{array}{l}R V_{t m a} \\
\left.a d j^{n}\right)\end{array}$ & $\begin{array}{l}R V_{t m a} \\
\left.\left.a d\right|^{2}\right)\end{array}$ & $\begin{array}{l}M R_{t} P \\
\text { ar }\end{array}$ & $M R_{t G}$ & $\begin{array}{l}M R_{t} R \\
\mathcal{S}\end{array}$ & $\begin{array}{l}M R_{t} Y \\
Z\end{array}$ & $\begin{array}{l}R R_{t} P \\
a r\end{array}$ & $\begin{array}{l}R R_{t} G \\
\kappa\end{array}$ & $\begin{array}{l}R R_{t} R \\
\mathcal{S}\end{array}$ & $\begin{array}{l}R R_{t Y} \\
Z\end{array}$ \\
\hline $116_{a c t}$ & $\begin{array}{l}6.60 e \\
-4\end{array}$ & $\begin{array}{c}5.09 e \\
-4\end{array}$ & $\begin{array}{l}9.27 e \\
-4\end{array}$ & $\begin{array}{l}9.27 e \\
-4\end{array}$ & $\begin{array}{l}1.70 \mathrm{e} \\
-4\end{array}$ & $\begin{array}{c}3.52 e \\
-5\end{array}$ & $\begin{array}{c}3.28 \mathrm{e} \\
-5\end{array}$ & $\begin{array}{c}3.01 \mathrm{e} \\
-5\end{array}$ & $\begin{array}{c}9.98 \mathrm{e} \\
-4\end{array}$ & $\begin{array}{l}1.44 \mathrm{e} \\
-4\end{array}$ & $\begin{array}{c}6.64 e \\
-5\end{array}$ & $\begin{array}{c}5.61 \mathrm{e} \\
-4\end{array}$ \\
\hline $\begin{array}{l}116_{t h} \\
e\end{array}$ & $\begin{array}{l}0.005 \\
3\end{array}$ & $\begin{array}{c}8.07 e \\
-4\end{array}$ & $\begin{array}{l}0.004 \\
2\end{array}$ & $\begin{array}{l}0.004 \\
0\end{array}$ & $\begin{array}{l}0.001 \\
1\end{array}$ & $\begin{array}{c}4.86 \mathrm{e} \\
-5\end{array}$ & $\begin{array}{l}3.42 \mathrm{e} \\
-5\end{array}$ & $\begin{array}{c}3.13 e \\
-5\end{array}$ & $\begin{array}{l}0.005 \\
7\end{array}$ & $\begin{array}{l}0.006 \\
0\end{array}$ & $\begin{array}{l}0.005 \\
9\end{array}$ & $\begin{array}{l}0.006 \\
4\end{array}$ \\
\hline $\begin{array}{l}C 14 \\
a c t_{1}\end{array}$ & $\begin{array}{c}9.85 e \\
-4\end{array}$ & $\begin{array}{c}3.97 e \\
-4\end{array}$ & $\begin{array}{l}6.40 e \\
-4\end{array}$ & $\begin{array}{l}6.39 e \\
-4\end{array}$ & $\begin{array}{c}3.25 e \\
-4\end{array}$ & $\begin{array}{c}2.27 e \\
-5\end{array}$ & $\begin{array}{c}2.36 e \\
-5\end{array}$ & $\begin{array}{c}2.08 \mathrm{e} \\
-5\end{array}$ & $\begin{array}{c}5.37 e \\
-4\end{array}$ & $\begin{array}{c}8.21 \mathrm{e} \\
-4\end{array}$ & $\begin{array}{c}6.36 e \\
-4\end{array}$ & $\begin{array}{c}6.33 e \\
-4\end{array}$ \\
\hline $\begin{array}{l}C 14 t \\
h e_{1}\end{array}$ & $\begin{array}{c}4.33 e \\
-4\end{array}$ & $\begin{array}{l}6.50 e \\
-4\end{array}$ & $\begin{array}{l}7.03 e \\
-4\end{array}$ & $\begin{array}{l}6.88 \mathrm{e} \\
-4\end{array}$ & $\begin{array}{l}1.79 e \\
-4\end{array}$ & $\begin{array}{l}1.07 e \\
-5\end{array}$ & $\begin{array}{l}1.05 e \\
-5\end{array}$ & $\begin{array}{l}1.02 \mathrm{e} \\
-5\end{array}$ & $\begin{array}{l}1.89 e \\
-4\end{array}$ & $\begin{array}{c}2.43 e \\
-4\end{array}$ & $\begin{array}{c}2.13 e \\
-4\end{array}$ & $\begin{array}{c}2.79 \mathrm{e} \\
-4\end{array}$ \\
\hline $\begin{array}{l}C 14 \\
\text { act }_{2}\end{array}$ & $\begin{array}{l}0.003 \\
1\end{array}$ & $\begin{array}{c}3.65 e \\
-4\end{array}$ & $\begin{array}{l}0.001 \\
8\end{array}$ & $\begin{array}{l}0.001 \\
8\end{array}$ & $\begin{array}{l}0.001 \\
5\end{array}$ & $\begin{array}{c}4.62 e \\
-5\end{array}$ & $\begin{array}{c}3.22 e \\
-5\end{array}$ & $\begin{array}{c}2.93 e \\
-5\end{array}$ & $\begin{array}{l}0.004 \\
2\end{array}$ & $\begin{array}{l}0.004 \\
3\end{array}$ & $\begin{array}{l}0.003 \\
2\end{array}$ & $\begin{array}{l}0.002 \\
4\end{array}$ \\
\hline $\begin{array}{l}C 14 \\
\text { act }_{2}\end{array}$ & $\begin{array}{c}2.95 e \\
-4\end{array}$ & $\begin{array}{c}5.53 e \\
-4\end{array}$ & $\begin{array}{l}8.95 e \\
-4\end{array}$ & $\begin{array}{l}7.95 e \\
-4\end{array}$ & $\begin{array}{l}1.68 \mathrm{e} \\
-4\end{array}$ & $\begin{array}{l}1.40 \mathrm{e} \\
-5\end{array}$ & $\begin{array}{l}1.32 \mathrm{e} \\
-5\end{array}$ & $\begin{array}{l}1.25 \mathrm{e} \\
-5\end{array}$ & $\begin{array}{l}1.95 e \\
-4\end{array}$ & $\begin{array}{l}1.95 e \\
-4\end{array}$ & $\begin{array}{l}1.43 e \\
-4\end{array}$ & $\begin{array}{c}3.57 e \\
-4\end{array}$ \\
\hline $\begin{array}{l}\text { C15 } \\
\text { act }\end{array}$ & $\begin{array}{l}0.032 \\
1\end{array}$ & $\begin{array}{l}0.002 \\
0\end{array}$ & $\begin{array}{l}0.057 \\
4\end{array}$ & $\begin{array}{l}0.060 \\
9\end{array}$ & $\begin{array}{l}0.015 \\
1\end{array}$ & $\begin{array}{c}3.75 e \\
-5\end{array}$ & $\begin{array}{c}3.33 e \\
-5\end{array}$ & $\begin{array}{c}3.05 e \\
-5\end{array}$ & $\begin{array}{l}0.004 \\
7\end{array}$ & $\begin{array}{l}0.005 \\
0\end{array}$ & $\begin{array}{l}0.004 \\
3\end{array}$ & $\begin{array}{l}0.003 \\
4\end{array}$ \\
\hline${ }_{h e}^{C 15_{t}}$ & $\begin{array}{c}3.50 \mathrm{e} \\
-4\end{array}$ & $\begin{array}{l}0.002 \\
1\end{array}$ & $\begin{array}{l}0.039 \\
0\end{array}$ & $\begin{array}{l}0.045 \\
5\end{array}$ & $\begin{array}{c}2.34 \mathrm{e} \\
-4\end{array}$ & $\begin{array}{c}2.56 \mathrm{e} \\
-5\end{array}$ & $\begin{array}{c}2.39 e \\
-5\end{array}$ & $\begin{array}{c}2.10 \mathrm{e} \\
-5\end{array}$ & $\begin{array}{c}9.16 \mathrm{e} \\
-5\end{array}$ & $\begin{array}{l}1.01 \mathrm{e} \\
-4\end{array}$ & $\begin{array}{l}1.22 \mathrm{e} \\
-4\end{array}$ & $\begin{array}{l}2.72 \mathrm{e} \\
-4\end{array}$ \\
\hline
\end{tabular}

Notes. Table 8 reports the mean magnitude of jumps $(\overline{J M})$ for both actual and theoretical BRIC Eurobond prices. $\overline{\overline{J M}}$ estimates are split into three groups: realized volatility, monthly range, and realized range estimates. All average values reported, are t-test significant in a $5 \%$ significance level.

Table 9.

Average ratio of magnitude of the jump component of risk to the magnitude of the continuous component $\overline{d R}$ ).

\begin{tabular}{|c|c|c|c|c|c|c|c|c|c|c|c|c|}
\hline & $\begin{array}{l}R V_{t} \\
m\end{array}$ & $\begin{array}{l}B P V_{t} \\
m\end{array}$ & $\begin{array}{l}R V_{t m a} a \\
d^{\prime j)}\end{array}$ & $\begin{array}{l}R V_{t m a} a \\
d f^{2}\end{array}$ & $\begin{array}{l}M R_{t} P \\
a r\end{array}$ & $\begin{array}{l}M R_{t} \\
G K\end{array}$ & $\begin{array}{l}M R_{t} \\
R S\end{array}$ & $\begin{array}{l}M R_{t} \\
Y Z\end{array}$ & $\begin{array}{l}R R t P \\
\text { ar }\end{array}$ & $\begin{array}{l}R R t \\
G K\end{array}$ & $\begin{array}{l}R R t R \\
S\end{array}$ & $\begin{array}{l}R R A Y \\
Z\end{array}$ \\
\hline$B 14_{a c t}$ & $\begin{array}{l}0.48 \\
04\end{array}$ & $\begin{array}{l}10.4 \\
8\end{array}$ & 0.7474 & 0.7474 & $\begin{array}{l}0.700 \\
7\end{array}$ & 1.43 & 1.24 & 1.29 & 1.08 & 1.14 & 1.33 & $\begin{array}{l}0.51 \\
61\end{array}$ \\
\hline$B 14_{\text {the }}$ & 2.33 & 9.07 & 2.02 & 1.87 & 2.60 & $\begin{array}{l}0.44 \\
52\end{array}$ & 0 & 0 & 2.56 & 4.01 & 6.27 & 1.36 \\
\hline$B 11_{a c t}$ & 1.52 & 5.04 & 0.8948 & 0.8788 & $\begin{array}{l}0.684 \\
9\end{array}$ & 1.73 & 1.76 & 1.55 & 1.02 & 8.21 & 5.02 & 1.22 \\
\hline$B 11_{\text {the }}$ & 5.47 & 3.06 & 2.46 & 2.49 & 1.88 & 1.85 & 1.06 & 1.46 & 5.10 & 5.10 & 5.10 & 5.10 \\
\hline$B 12_{a c t}$ & 1.84 & 7.51 & 0.4638 & 0.4261 & $\begin{array}{l}0.797 \\
4\end{array}$ & $\begin{array}{l}0.95 \\
87\end{array}$ & $\begin{array}{l}0.90 \\
84\end{array}$ & 1.27 & 1.65 & 1.83 & 1.43 & 1.69 \\
\hline$B 12_{\text {the }}$ & 3.98 & $\begin{array}{l}14.1 \\
6\end{array}$ & 1.58 & 1.69 & 1.73 & 1.61 & 1.39 & 1.64 & 5.87 & 5.87 & 5.87 & 5.87 \\
\hline$R 13_{a c t}$ & 1.22 & $\begin{array}{l}16.3 \\
3\end{array}$ & 0.4443 & 0.4688 & 1.62 & $\begin{array}{l}0.75 \\
31\end{array}$ & $\begin{array}{l}0.65 \\
38\end{array}$ & $\begin{array}{l}0.52 \\
43\end{array}$ & $\begin{array}{l}0.67 \\
94\end{array}$ & $\begin{array}{l}0.71 \\
49\end{array}$ & 1.11 & $\begin{array}{l}0.57 \\
93\end{array}$ \\
\hline$R 13_{\text {the }}$ & 2.86 & 6.11 & 2.01 & 1.97 & 4.04 & $\begin{array}{l}0.22 \\
49\end{array}$ & 0 & 0 & 6.18 & 6.43 & 4.62 & 1.63 \\
\hline $\begin{array}{l}R 10 a \\
c t_{1}\end{array}$ & $\begin{array}{l}0.86 \\
22\end{array}$ & $\begin{array}{l}22.2 \\
5\end{array}$ & 1.03 & 1.06 & 2.25 & 1.83 & 1.61 & 1.72 & 7.91 & $\begin{array}{l}32.9 \\
3\end{array}$ & $\begin{array}{l}26.6 \\
1\end{array}$ & $\begin{array}{l}32.5 \\
9\end{array}$ \\
\hline $\begin{array}{l}R 10 \text { th } \\
e_{1}\end{array}$ & 2.33 & 5.71 & 1.78 & 1.54 & 1.27 & $\begin{array}{l}0.99 \\
34\end{array}$ & $\begin{array}{l}0.75 \\
03\end{array}$ & $\begin{array}{l}0.75 \\
57\end{array}$ & 2.01 & $\begin{array}{l}12.0 \\
2\end{array}$ & $\begin{array}{l}14.9 \\
4\end{array}$ & 17.3 \\
\hline$R 10 a$ & 1.42 & 15.5 & 0.8640 & 1.05 & 0.743 & 1.58 & 1.05 & 0.89 & 1.21 & 1.26 & 2.22 & 1.54 \\
\hline
\end{tabular}




\begin{tabular}{|c|c|c|c|c|c|c|c|c|c|c|c|c|}
\hline & $\begin{array}{l}R V_{t} \\
m\end{array}$ & $\begin{array}{l}B P V_{t} \\
m\end{array}$ & $\begin{array}{l}R V_{t m a} a \\
d j^{\prime \prime}\end{array}$ & $\begin{array}{l}R V t m a \text { a } \\
d f^{2)}\end{array}$ & $\begin{array}{l}M R_{t P} \\
a r\end{array}$ & $\begin{array}{l}M R t \\
G K\end{array}$ & $\begin{array}{l}M R_{t} \\
R S\end{array}$ & $\begin{array}{l}M R t \\
Y Z\end{array}$ & $\begin{array}{l}R R_{t P} \\
a r\end{array}$ & $\begin{array}{l}R R_{t} \\
G K\end{array}$ & $\begin{array}{l}R R_{t R} \\
S\end{array}$ & $\begin{array}{l}R R t Y \\
Z\end{array}$ \\
\hline$c t_{2}$ & & 6 & & & 7 & & & 14 & & & & \\
\hline $\begin{array}{l}R 10_{\text {th }} \\
e_{2}\end{array}$ & 2.89 & 5.26 & 2.16 & 1.67 & 1.30 & 1.07 & $\begin{array}{l}0.65 \\
71\end{array}$ & $\begin{array}{l}0.66 \\
52\end{array}$ & 2.07 & 2.17 & 1.66 & 2.60 \\
\hline $116_{a c t}$ & 1.22 & 7.38 & 1.14 & 1.14 & $\begin{array}{l}0.858 \\
9\end{array}$ & 2.63 & $\begin{array}{l}18.0 \\
0\end{array}$ & $\begin{array}{l}16.5 \\
1\end{array}$ & 2.47 & 1.03 & 1.06 & 1.27 \\
\hline $116_{\text {the }}$ & $\begin{array}{l}13.1 \\
5\end{array}$ & 8.66 & 2.96 & 2.79 & 3.50 & $\begin{array}{l}15.1 \\
1\end{array}$ & $\begin{array}{l}10.6 \\
5\end{array}$ & 9.74 & $\begin{array}{l}13.2 \\
0\end{array}$ & $\begin{array}{l}13.7 \\
9\end{array}$ & $\begin{array}{l}10.4 \\
5\end{array}$ & $\begin{array}{l}14.3 \\
4\end{array}$ \\
\hline $\begin{array}{l}C 14 a \\
c t_{1}\end{array}$ & 1.69 & 5.63 & 1.23 & 1.23 & 1.35 & $\begin{array}{l}0.87 \\
54\end{array}$ & 1.91 & 1.68 & 1.21 & 1.36 & $\begin{array}{l}0.85 \\
20\end{array}$ & 1.58 \\
\hline $\begin{array}{l}\text { C14th } \\
e_{1}\end{array}$ & 2.12 & 8.71 & 0.7765 & 0.7739 & $\begin{array}{l}0.877 \\
1\end{array}$ & $\begin{array}{l}0.28 \\
45\end{array}$ & 0 & 0 & 1.18 & 1.38 & 1.73 & $\begin{array}{l}0.67 \\
20\end{array}$ \\
\hline $\begin{array}{l}C 14 a \\
c t_{2}\end{array}$ & 8.02 & 5.32 & 3.64 & 3.61 & 4.50 & $\begin{array}{l}10.7 \\
8\end{array}$ & 7.51 & 6.85 & $\begin{array}{l}14.7 \\
0\end{array}$ & $\begin{array}{l}15.2 \\
7\end{array}$ & $\begin{array}{l}11.2 \\
8\end{array}$ & 7.21 \\
\hline $\begin{array}{l}C 14 a \\
c t_{2}\end{array}$ & $\begin{array}{l}0.73 \\
41\end{array}$ & 7.95 & 1.09 & 0.9977 & 1.21 & $\begin{array}{l}0.39 \\
94\end{array}$ & 0 & 0 & 1.16 & 1.16 & 1.09 & $\begin{array}{l}0.51 \\
76\end{array}$ \\
\hline$C 15_{a c t}$ & $\begin{array}{l}53.2 \\
6\end{array}$ & $\begin{array}{l}16.0 \\
6\end{array}$ & 2.06 & 2.18 & 32.92 & 2.93 & $\begin{array}{l}10.7 \\
6\end{array}$ & 9.84 & 4.00 & 4.16 & 2.73 & 3.65 \\
\hline$C 15_{\text {the }}$ & $\begin{array}{l}0.82 \\
22\end{array}$ & $\begin{array}{l}17.6 \\
6\end{array}$ & 1.25 & 1.46 & $\begin{array}{l}0.994 \\
0\end{array}$ & 1.02 & 1.87 & 1.64 & $\begin{array}{l}0.33 \\
27\end{array}$ & $\begin{array}{l}0.36 \\
67\end{array}$ & 0 & $\begin{array}{l}0.88 \\
76\end{array}$ \\
\hline
\end{tabular}

Notes. Table 9 reports the average ratio of magnitude of the jump component of risk to the magnitude of the continuous component $(\bar{R}$ ) for both actual and theoretical BRIC Eurobond prices. $\overline{J R}$ estimates are split into three groups: realized volatility, monthly range, and realized range estimates. All average values reported, are t-test significant in a $5 \%$ significance level.

Table 10.

Average frequency of jump occurrence $\overline{\mathcal{L}}$ ).

\begin{tabular}{|c|c|c|c|c|c|c|c|c|c|c|c|c|}
\hline & $\begin{array}{l}R V_{t} \\
m\end{array}$ & $\begin{array}{l}B P V_{t} \\
m\end{array}$ & $\begin{array}{l}R V_{t m a} a \\
d j^{\prime)}\end{array}$ & $\begin{array}{l}R V_{t m a} \text { a } \\
d f^{2)}\end{array}$ & $\begin{array}{l}M R t P \\
a r\end{array}$ & $\begin{array}{l}M R_{t} \\
G \kappa\end{array}$ & $\begin{array}{l}M R t \\
R S\end{array}$ & $\begin{array}{l}M R t \\
Y Z\end{array}$ & $\begin{array}{l}R R_{t P} \\
a r\end{array}$ & $\begin{array}{l}R R_{t} \\
G K\end{array}$ & $\begin{array}{l}R R_{t} R \\
S\end{array}$ & $\begin{array}{l}R R t Y \\
Z\end{array}$ \\
\hline$B 14_{a c t}$ & $\begin{array}{l}0.41 \\
67\end{array}$ & $\begin{array}{l}0.91 \\
67 \square\end{array}$ & $\begin{array}{l}0.5000 \\
\square\end{array}$ & $\begin{array}{l}0.5000 \\
\square\end{array}$ & $\begin{array}{l}0.333 \\
3\end{array}$ & $\begin{array}{l}0.22 \\
22\end{array}$ & $\begin{array}{l}0.16 \\
67\end{array}$ & $\begin{array}{l}0.13 \\
89\end{array}$ & $\begin{array}{l}0.27 \\
78\end{array}$ & $\begin{array}{l}0.30 \\
56\end{array}$ & $\begin{array}{l}0.22 \\
22\end{array}$ & $\begin{array}{l}0.41 \\
67\end{array}$ \\
\hline$B 14_{\text {the }}$ & $\begin{array}{l}0.36 \\
11\end{array}$ & $1.00 \unrhd$ & $\begin{array}{l}0.5556 \\
\square\end{array}$ & $\begin{array}{l}0.5556 \\
\square\end{array}$ & $\begin{array}{l}0.250 \\
0\end{array}$ & $\begin{array}{l}0.02 \\
78\end{array}$ & $\begin{array}{l}0.02 \\
78\end{array}$ & $\begin{array}{l}0.02 \\
78\end{array}$ & $\begin{array}{l}0.13 \\
89\end{array}$ & $\begin{array}{l}0.11 \\
11\end{array}$ & $\begin{array}{l}0.08 \\
33\end{array}$ & $\begin{array}{l}0.27 \\
78\end{array}$ \\
\hline$B 11_{a c t}$ & $\begin{array}{l}0.38 \\
89\end{array}$ & $\begin{array}{l}0.77 \\
78 \square\end{array}$ & $\begin{array}{l}0.8056 \\
\square\end{array}$ & $\begin{array}{l}0.8056 \\
\square\end{array}$ & $\begin{array}{l}0.444 \\
4\end{array}$ & $\begin{array}{l}0.41 \\
67\end{array}$ & $\begin{array}{l}0.33 \\
33\end{array}$ & $\begin{array}{l}0.33 \\
33\end{array}$ & $\begin{array}{l}0.41 \\
67\end{array}$ & $\begin{array}{l}0.66 \\
67 \square\end{array}$ & $\begin{array}{l}0.25 \\
00\end{array}$ & $\begin{array}{l}0.77 \\
78 \unrhd\end{array}$ \\
\hline$B 11_{\text {the }}$ & $\begin{array}{l}0.25 \\
00\end{array}$ & $\begin{array}{l}0.88 \\
89 \square\end{array}$ & $\begin{array}{l}0.5000 \\
\square\end{array}$ & $\begin{array}{l}0.5000 \\
\square\end{array}$ & $\begin{array}{l}0.361 \\
1\end{array}$ & $\begin{array}{l}0.25 \\
00\end{array}$ & $\begin{array}{l}0.25 \\
00\end{array}$ & $\begin{array}{l}0.19 \\
44\end{array}$ & $\begin{array}{l}0.47 \\
22\end{array}$ & $\begin{array}{l}0.47 \\
22\end{array}$ & $\begin{array}{l}0.47 \\
22\end{array}$ & $\begin{array}{l}0.47 \\
22\end{array}$ \\
\hline$B 12_{a c t}$ & $\begin{array}{l}0.36 \\
11\end{array}$ & $\begin{array}{l}0.86 \\
11 \square\end{array}$ & $\begin{array}{l}0.6111 \\
\square\end{array}$ & $\begin{array}{l}0.6389 \\
\square\end{array}$ & $\begin{array}{l}0.361 \\
1\end{array}$ & $\begin{array}{l}0.50 \\
00 \square\end{array}$ & $\begin{array}{l}0.33 \\
33\end{array}$ & $\begin{array}{l}0.25 \\
00\end{array}$ & $\begin{array}{l}0.63 \\
89 \square\end{array}$ & $\begin{array}{l}0.52 \\
78 \square\end{array}$ & $\begin{array}{l}0.41 \\
67\end{array}$ & $\begin{array}{l}0.88 \\
89 \square\end{array}$ \\
\hline$B 12_{\text {the }}$ & $\begin{array}{l}0.25 \\
00\end{array}$ & $\begin{array}{l}0.86 \\
11 \square\end{array}$ & $\begin{array}{l}0.5556 \\
\square\end{array}$ & $\begin{array}{l}0.5556 \\
\square\end{array}$ & $\begin{array}{l}0.277 \\
8\end{array}$ & $\begin{array}{l}0.27 \\
78\end{array}$ & $\begin{array}{l}0.22 \\
22\end{array}$ & $\begin{array}{l}0.19 \\
44\end{array}$ & $\begin{array}{l}0.50 \\
00 \unrhd\end{array}$ & $\begin{array}{l}0.50 \\
00 \square\end{array}$ & $\begin{array}{l}0.50 \\
00 \unrhd\end{array}$ & $\begin{array}{l}0.50 \\
00 \unrhd\end{array}$ \\
\hline$R 13_{a c t}$ & $\begin{array}{l}0.33 \\
33\end{array}$ & $\begin{array}{l}0.94 \\
44 \square\end{array}$ & 0.4444 & 0.4722 & $\begin{array}{l}0.611 \\
1 \square\end{array}$ & $\begin{array}{l}0.33 \\
33\end{array}$ & $\begin{array}{l}0.16 \\
67\end{array}$ & $\begin{array}{l}0.16 \\
67\end{array}$ & $\begin{array}{l}0.30 \\
56\end{array}$ & $\begin{array}{l}0.22 \\
22\end{array}$ & $\begin{array}{l}0.19 \\
44\end{array}$ & $\begin{array}{l}0.69 \\
44 \square\end{array}$ \\
\hline$R 13_{\text {the }}$ & $\begin{array}{l}0.25 \\
00\end{array}$ & $\begin{array}{l}1.00 \\
\square\end{array}$ & $\begin{array}{l}0.6389 \\
\square\end{array}$ & $\begin{array}{l}0.6667 \\
\square\end{array}$ & $\begin{array}{l}0.166 \\
7\end{array}$ & $\begin{array}{l}0.08 \\
33\end{array}$ & $\begin{array}{l}0.05 \\
55\end{array}$ & $\begin{array}{l}0.05 \\
55\end{array}$ & $\begin{array}{l}0.11 \\
11\end{array}$ & $\begin{array}{l}0.11 \\
11\end{array}$ & $\begin{array}{l}0.11 \\
11\end{array}$ & $\begin{array}{l}0.61 \\
11 \square\end{array}$ \\
\hline
\end{tabular}




\begin{tabular}{|c|c|c|c|c|c|c|c|c|c|c|c|c|}
\hline & $\begin{array}{l}R V_{t} \\
m\end{array}$ & $\begin{array}{l}B P V_{t} \\
m\end{array}$ & $\begin{array}{l}R V_{t m a} a \\
\left.d f^{\prime \prime}\right)\end{array}$ & $\begin{array}{l}R V_{t m a} a \\
d^{\left.d{ }^{2}\right)}\end{array}$ & $\begin{array}{l}M R_{t} P \\
\text { ar }\end{array}$ & $\begin{array}{l}M R t \\
G K\end{array}$ & $\begin{array}{l}M R t \\
R S\end{array}$ & $\begin{array}{l}M R_{t} \\
Y Z\end{array}$ & $\begin{array}{l}R R_{t} P \\
\text { ar }\end{array}$ & $\begin{array}{l}R R_{t} \\
G K\end{array}$ & $\begin{array}{l}R R_{t R} \\
\mathcal{S}\end{array}$ & $\begin{array}{l}R R_{t} Y \\
Z\end{array}$ \\
\hline $\begin{array}{l}R 10 a \\
c t_{1}\end{array}$ & $\begin{array}{l}0.41 \\
67\end{array}$ & $\begin{array}{l}0.80 \\
56 \square\end{array}$ & $\begin{array}{l}0.5833 \\
\square\end{array}$ & $\begin{array}{l}0.5833 \\
\square\end{array}$ & $\begin{array}{l}0.527 \\
8 \square\end{array}$ & $\begin{array}{l}0.38 \\
89\end{array}$ & $\begin{array}{l}0.33 \\
33\end{array}$ & $\begin{array}{l}0.30 \\
56\end{array}$ & $\begin{array}{l}0.41 \\
67\end{array}$ & $\begin{array}{l}0.88 \\
89 \square\end{array}$ & $\begin{array}{l}0.88 \\
89 \square\end{array}$ & $\begin{array}{l}0.91 \\
67 \unrhd\end{array}$ \\
\hline $\begin{array}{l}R 10_{\text {th }} \\
e_{1}\end{array}$ & $\begin{array}{l}0.30 \\
56\end{array}$ & $\begin{array}{l}0.88 \\
89 \square\end{array}$ & $\begin{array}{l}0.6944 \\
\square\end{array}$ & $\begin{array}{l}0.7222 \\
\square\end{array}$ & $\begin{array}{l}0.222 \\
2\end{array}$ & $\begin{array}{l}0.27 \\
78\end{array}$ & $\begin{array}{l}0.22 \\
22\end{array}$ & $\begin{array}{l}0.19 \\
44\end{array}$ & $\begin{array}{l}0.30 \\
56\end{array}$ & $\begin{array}{l}0.97 \\
22 \square\end{array}$ & $\begin{array}{l}0.94 \\
44 \square\end{array}$ & $\begin{array}{l}0.97 \\
22 \square\end{array}$ \\
\hline $\begin{array}{l}R 10 a \\
c t_{2}\end{array}$ & $\begin{array}{l}0.44 \\
44\end{array}$ & $\begin{array}{l}0.69 \\
44 \square\end{array}$ & $\begin{array}{l}0.5556 \\
\square\end{array}$ & $\begin{array}{l}0.5550 \\
\square\end{array}$ & $\begin{array}{l}0.500 \\
0 \unrhd\end{array}$ & $\begin{array}{l}0.58 \\
33 \square\end{array}$ & $\begin{array}{l}0.52 \\
78 \unrhd\end{array}$ & $\begin{array}{l}0.52 \\
78 \unrhd\end{array}$ & $\begin{array}{l}0.72 \\
22 \square\end{array}$ & $\begin{array}{l}0.75 \\
00 \unrhd\end{array}$ & $\begin{array}{l}0.50 \\
00 \unrhd\end{array}$ & $\begin{array}{l}0.91 \\
67 \square\end{array}$ \\
\hline $\begin{array}{l}R 10_{\text {th }} \\
e_{2}\end{array}$ & $\begin{array}{l}0.27 \\
78\end{array}$ & $\begin{array}{l}0.91 \\
67 \square\end{array}$ & $\begin{array}{l}0.6944 \\
\square\end{array}$ & $\begin{array}{l}0.7222 \\
\square\end{array}$ & $\begin{array}{l}0.222 \\
2\end{array}$ & $\begin{array}{l}0.22 \\
22\end{array}$ & $\begin{array}{l}0.19 \\
44\end{array}$ & $\begin{array}{l}0.16 \\
67\end{array}$ & $\begin{array}{l}0.44 \\
44\end{array}$ & $\begin{array}{l}0.44 \\
44\end{array}$ & $\begin{array}{l}0.33 \\
33\end{array}$ & $\begin{array}{l}0.63 \\
89 \square\end{array}$ \\
\hline $116_{a}$ & $\begin{array}{l}0.25 \\
00\end{array}$ & $\begin{array}{l}0.91 \\
67 \square\end{array}$ & $\begin{array}{l}0.6111 \\
\square\end{array}$ & $\begin{array}{l}0.6111 \\
\square\end{array}$ & $\begin{array}{l}0.305 \\
6\end{array}$ & $\begin{array}{l}0.08 \\
33\end{array}$ & $\begin{array}{l}0.05 \\
56\end{array}$ & $\begin{array}{l}0.05 \\
56\end{array}$ & $\begin{array}{l}0.11 \\
11\end{array}$ & $\begin{array}{l}0.11 \\
11\end{array}$ & $\begin{array}{l}0.11 \\
11\end{array}$ & $\begin{array}{l}0.30 \\
56\end{array}$ \\
\hline 116 & $\begin{array}{l}0.19 \\
44\end{array}$ & $\begin{array}{l}0.97 \\
22 \square\end{array}$ & $\begin{array}{l}0.5278 \\
\square\end{array}$ & $\begin{array}{l}0.5278 \\
\square\end{array}$ & $\begin{array}{l}0.333 \\
3\end{array}$ & $\begin{array}{l}0.02 \\
78\end{array}$ & $\begin{array}{l}0.02 \\
78\end{array}$ & $\begin{array}{l}0.02 \\
78\end{array}$ & $\begin{array}{l}0.11 \\
11\end{array}$ & $\begin{array}{l}0.11 \\
11\end{array}$ & $\begin{array}{l}0.08 \\
33\end{array}$ & $\begin{array}{l}0.16 \\
67\end{array}$ \\
\hline $\begin{array}{l}C 14 a \\
c t_{1}\end{array}$ & $\begin{array}{l}0.36 \\
11\end{array}$ & $1.00 \square$ & $\begin{array}{l}0.7222 \\
\square\end{array}$ & $\begin{array}{l}0.7222 \\
\square\end{array}$ & $\begin{array}{l}0.250 \\
0\end{array}$ & $\begin{array}{l}0.05 \\
56\end{array}$ & $\begin{array}{l}0.02 \\
78\end{array}$ & $\begin{array}{l}0.02 \\
78\end{array}$ & $\begin{array}{l}0.33 \\
33\end{array}$ & $\begin{array}{l}0.22 \\
22\end{array}$ & $\begin{array}{l}0.16 \\
67\end{array}$ & $\begin{array}{l}0.72 \\
22 \square\end{array}$ \\
\hline $\begin{array}{l}\text { C14 th } \\
e_{1}\end{array}$ & $\begin{array}{l}0.13 \\
89\end{array}$ & $1.00 \unrhd$ & $\begin{array}{l}0.7222 \\
\square\end{array}$ & $\begin{array}{l}0.7500 \\
\square\end{array}$ & $\begin{array}{l}0.333 \\
3\end{array}$ & $\begin{array}{l}0.02 \\
78\end{array}$ & $\begin{array}{l}0.02 \\
78\end{array}$ & $\begin{array}{l}0.02 \\
78\end{array}$ & $\begin{array}{l}0.22 \\
22\end{array}$ & $\begin{array}{l}0.16 \\
67\end{array}$ & $\begin{array}{l}0.11 \\
11\end{array}$ & $\begin{array}{l}0.63 \\
89 \square\end{array}$ \\
\hline $\begin{array}{l}C 14 a \\
c t_{2}\end{array}$ & $\begin{array}{l}0.30 \\
56\end{array}$ & $\begin{array}{l}0.97 \\
22 \square\end{array}$ & $\begin{array}{l}0.7500 \\
\square\end{array}$ & $\begin{array}{l}0.7500 \\
\square\end{array}$ & $\begin{array}{l}0.277 \\
8\end{array}$ & $\begin{array}{l}0.02 \\
78\end{array}$ & $\begin{array}{l}0.02 \\
78\end{array}$ & $\begin{array}{l}0.02 \\
78\end{array}$ & $\begin{array}{l}0.13 \\
89\end{array}$ & $\begin{array}{l}0.13 \\
89\end{array}$ & $\begin{array}{l}0.13 \\
89\end{array}$ & $\begin{array}{l}0.41 \\
67\end{array}$ \\
\hline $\begin{array}{l}C 14 a \\
c t_{2}\end{array}$ & $\begin{array}{l}0.25 \\
00\end{array}$ & $1.00 \square$ & $\begin{array}{l}0.6667 \\
\square\end{array}$ & $\begin{array}{l}0.6944 \\
\square\end{array}$ & $\begin{array}{l}0.250 \\
0\end{array}$ & $\begin{array}{l}0.02 \\
78\end{array}$ & $\begin{array}{l}0.02 \\
78\end{array}$ & $\begin{array}{l}0.02 \\
78\end{array}$ & $\begin{array}{l}0.08 \\
33\end{array}$ & $\begin{array}{l}0.08 \\
33\end{array}$ & $\begin{array}{l}0.05 \\
56\end{array}$ & $\begin{array}{l}0.38 \\
89\end{array}$ \\
\hline$C 15_{a c t}$ & $\begin{array}{l}0.44 \\
44\end{array}$ & $\begin{array}{l}0.91 \\
67 \square\end{array}$ & $\begin{array}{l}0.7222 \\
\square\end{array}$ & $\begin{array}{l}0.7222 \\
\square\end{array}$ & $\begin{array}{l}0.388 \\
9\end{array}$ & $\begin{array}{l}0.08 \\
33\end{array}$ & $\begin{array}{l}0.05 \\
56\end{array}$ & $\begin{array}{l}0.05 \\
56\end{array}$ & $\begin{array}{l}0.14 \\
29\end{array}$ & $\begin{array}{l}0.14 \\
29\end{array}$ & $\begin{array}{l}0.11 \\
11\end{array}$ & $\begin{array}{l}0.35 \\
71\end{array}$ \\
\hline$C 15_{\text {the }}$ & $\begin{array}{l}0.19 \\
44\end{array}$ & $1.00 \unrhd$ & $\begin{array}{l}0.6667 \\
\square\end{array}$ & $\begin{array}{l}0.6667 \\
\square\end{array}$ & $\begin{array}{l}0.277 \\
8\end{array}$ & $\begin{array}{l}0.05 \\
56\end{array}$ & $\begin{array}{l}0.02 \\
78\end{array}$ & $\begin{array}{l}0.02 \\
78\end{array}$ & $\begin{array}{l}0.08 \\
33\end{array}$ & $\begin{array}{l}0.08 \\
33\end{array}$ & $\begin{array}{l}0.08 \\
33\end{array}$ & $\begin{array}{l}0.16 \\
67\end{array}$ \\
\hline
\end{tabular}

Notes. Table 10 reports the average frequency of jump occurrence $\left(\bar{J}_{)}\right.$for both actual and theoretical BRIC Eurobond prices. $\overline{\bar{J}}$ estimates are split into three groups: realized volatility, monthly range and realized range estimates. All average values reported, are t-test significant in a $5 \%$ significance level.

Indicates significance if the average frequency of jump occurre $\bar{h}$ ce ( ) is higher than $50 \%$.

Table 11.

Theoretical vs actual prices.

\begin{tabular}{|c|c|c|c|c|c|}
\hline & $\bar{R}$ & $\overline{S R}$ & $\overline{J M}$ & $\overline{J R}$ & $\bar{J}$ \\
\hline$R V$ & $60^{\circ}$ & $50 \%$ & $63 \%$ & $68 \%$ & $43 \%$ \\
\hline & & & & 20 & \\
\hline & $55 \%$ & $8 \%$ & $55 \%$ & $60 \%$ & $5 \%$ \\
\hline
\end{tabular}

Notes. Table 11 reports the average percentage of bonds for which a risk or jump estimate $(\bar{R}, \overline{S R}, \overline{J M}, \overline{J R}$ and $\bar{J})$ is higher for theoretical prices rather than for actual prices.

Table 12.

Summarized results for BRIC countries.

\begin{tabular}{|c|c|c|c|c|c|c|}
\hline \multicolumn{2}{|r|}{$\bar{R}$} & $\overline{S R}$ & $\overline{J M}$ & $\overline{J R}$ & $\bar{J}$ & $J^{*}$ \\
\hline Brazil & $B P V_{t m} / M R_{t} Y Z$ & $B P V_{t m} / M R_{t} Y Z$ & $R R_{t} G K / M R_{t} Y Z$ & $B P V_{t m} / M R_{t R S}$ & $B P V_{t m} / M R_{t} Y Z$ & $39 \%$ \\
\hline Russia & $B P V_{t m} / M R_{t} Y Z$ & $M R_{t} Y Z / B P V_{t m}$ & $R R_{t} Y Z I M R_{t} Y Z$ & $B P V_{t m} / M R_{t R S}$ & $B P V_{t m /} / M R_{t} Y Z$ & $49 \%$ \\
\hline
\end{tabular}




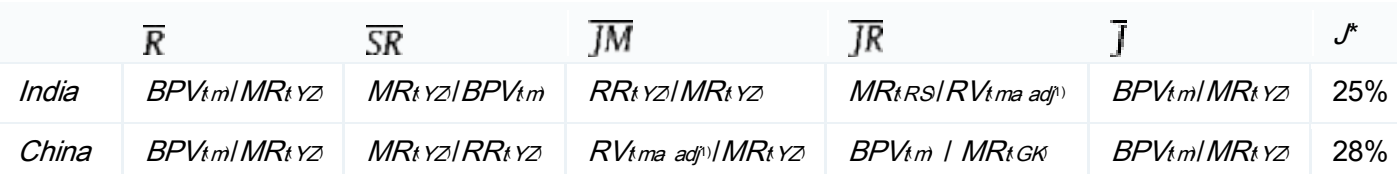

Notes. Table 12 reports the estimators with the highest and (/) lowest values for the corresponding risk and jump measures $(\bar{R}, \overline{S R}, \overline{J M}, \overline{J R}$ and $\bar{J})$. The last column provides the percentage of bonds (across estimators) for which the frequency of occurrence of significant jumps is higher than $50 \%(J)$.

Table 13.

Summarized results for groups of estimators.

\begin{tabular}{|c|c|c|c|c|c|}
\hline \multicolumn{2}{|r|}{$\bar{R}$} & $\overline{S R}$ & $\overline{J M}$ & $\overline{J R}$ & $\bar{J}$ \\
\hline$R V$ & China/Brazil & China/Brazil & China/Brazil & China/Brazil & Brazi \\
\hline & Braz & Chin & Indi & Ind & $\mathrm{Ru}$ \\
\hline & China/Brazil & China/Brazil & India/Brazil & Russia/China & Rus \\
\hline
\end{tabular}

Notes. Table 13 reports the countries with the highest and (/) lowest risk and jump measures for each group of estimators.

\subsection{Unconditional distribution of return and volatility series}

Many papers have examined the unconditional distribution of realized volatility (see lllueca \& Lafuente, 2006 and Wang, Wu, \& Yang, 2008). Table 4 provides summary statistics for the unconditional distribution of the realized volatilities. Volatility series for most of the BRIC Eurobonds and for most of volatility estimators are skewed to the right (skewness higher than zero) and leptokurtic (higher than three).

Table 5 deploys results for normality testing. The normality (CVM and QQ) tests do reject the normality null hypothesis for most of the BRIC Eurobonds and across the board of estimators. The critical value derived under independence for the CVM-test is $0.458(5 \%)$; and for the QQ-test is: $37.65(5 \%)$. Most of volatilities (regardless either the group of estimators or the country they belong to) are not normally distributed. The null hypothesis of normality is not rejected for the MR(GK),MR(RS) and MR(YZ estimators. All results for the skewness and kurtosis as well as for the normality testing of the unconditional distribution of volatilities are consistent for both actual and theoretical prices.

\subsection{Risk}

Risk is measured via the mean of magnitude of risk $\bar{R})$ (Table 6) and the mean of Sharpe ratios $(\overline{S R})($ Table 7$)$.

\subsubsection{Average magnitude of risk $(\bar{R})$}

The realized volatility $(R V)$ group of estimators has the highest mean magnitude of risk series $(\bar{R})$ across all BRIC countries. The highest (lowest) mean of risk $\left(R_{t}\right)$ series $(\bar{R})$ comes from the $B P V_{(m)}(M R(Y Z))$ estimator across BRIC Eurobonds, whereas the highest mean of risk $\left(R_{t}\right)$ series $(\bar{R})$ comes from the Chinese Eurobonds across the board of estimators. 
For the group of realized volatility estimators $(R V)$ and the group of realized range-based volatility estimators $(R R)$, Chinese (Brazilian) Eurobonds have the highest (lowest) mean magnitude of risk $\left(R_{t}\right)$ series $\left.\bar{R}\right)$ among BRIC countries. For the group of monthly ranges $(M R$ ), Brazilian (Indian) Eurobonds have the highest (lowest) $\bar{R}$ among BRIC countries.

The $B P V^{(}(m)\left(R V k m a \cdot a d f^{2}\right)$ ) estimator has the highest (lowest) mean magnitude of risk $\left(R_{t}\right)$ series $(\bar{R})$ among the realized volatility estimators $(R V)$, across all BRIC Eurobonds. The MRAYZ (MR $\left(\mathrm{Par}^{\prime}\right)$ estimator has the highest (lowest) $\bar{R}$ among the monthly ranges (MR), across all BRIC Eurobonds. The $R R(R S)(R R(Y Z)$ estimator has the highest (lowest) $\bar{R}$ among the realized range-based volatility estimators $(R R)$, across all BRIC Eurobonds. Regarding the performance of risk estimators, via the mean magnitude of risk $\left(R_{t}\right)$ series $(\bar{R})$, results are consistent across all BRIC Eurobonds. All mean values of risk estimates for all bonds and estimators are t-test statistically significant.

Moreover, the mean magnitude of risk $\left(R_{t}\right)$ series $(\bar{R})$ coming from theoretical prices (theoretical-price risk) is higher than the mean risk coming from actual market prices (actualprice risk). This result is evident in most of estimators and BRIC bonds. Across most of the eurobonds, the higher the expiry period, the higher the mean magnitude of risk $(\bar{R})$ is.

\subsubsection{Average Sharpe ratio $(\overline{S R})$}

The monthly range group of estimators $(M R)$ has the highest mean of Sharpe ratios $\left(S R_{t}\right)$ series $(\overline{S R})$ across all BRIC countries. The highest (lowest) $\overline{S R}$ comes from

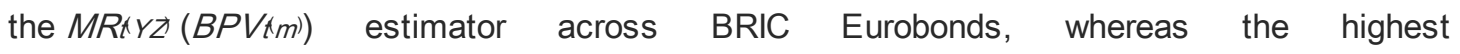
(lowest) $\overline{S R}$ comes from the Chinese (Brazilian) Eurobond across the board of estimators.

In specific, regarding Brazil, the $R V$ realized volatility ( $M R$ monthly range) group of estimators has the highest (lowest) mean of Sharpe ratios $\left(S R_{t}\right)$ series $\left.\overline{(R}\right)$ among groups, and the $\left.B P V_{t} m\right)(M R(Y Z) \quad$ estimator among individual estimators. Regarding Russia, the $M R$ monthly range ( $R V$ realized volatility) group of estimators has the highest (lowest) $\overline{S R}$ among groups, and the $M R(Y Z(B P V t m))$ estimator among individual estimators. Regarding India, the $M R$ monthly range ( $R V$ realized volatility) group of estimators has the highest (lowest) $\overline{S R}$ among groups, and the $M R \wedge Y Z(B P V k m)$ estimator among individual estimators. Regarding China, the $M R$ monthly range ( $R R$ realized range) group of estimators has the highest (lowest) $\overline{S R}$ among groups, and the $M R A Y Z(R R(Y Z)$ estimator among individual estimators.

For all three $(R V, M R$ and $R R)$ groups of estimators, Chinese (Brazilian) Eurobonds have the highest (lowest) mean of Sharpe ratios $\left(S R_{t}\right)$ series $(\overline{S R})$ among BRIC countries. The BPVtm) (RVtma ad $\left.{ }^{2}\right)$ ) estimator has the highest (lowest) mean of Sharpe ratios (SRt) series $(\overline{S R})$ among the realized volatility estimators, across all BRIC Eurobonds. The MRAPat (MR $(Y Z)$ ) estimator has the highest (lowest) $\overline{S R}$ among the monthly ranges, across all BRIC Eurobonds. The MRARS $\left(M R R^{\prime} G K\right)$ estimator has the highest (lowest) $\overline{S R}$ among the realized range-based volatility estimators, across all BRIC Eurobonds.

Regarding the mean of Sharpe ratios $\left(S R_{t}\right)$ series $(\overline{S R})$, all estimators are consistent and results change because of the informational content of BRIC bonds. All mean values of risk 
estimates for all bonds and estimators are t-test statistically significant. Moreover, the mean of Sharpe ratios $\left(S R_{t}\right)$ series $\left.\overline{S R}\right)$ coming from theoretical prices (theoretical-price Sharpe ratios) is higher than the mean Sharpe ratio coming from actual market prices (actual-price Sharpe ratio). This result is evident in most of estimators and BRIC bonds. Across most of the eurobonds, the higher the expiry period, the higher the mean magnitude of Sharpe ratios $\left(S R_{t}\right)$ series $(\overline{S R})$ is.

\subsection{Jumps}

Eraker, Johannes, and Polson (2003) as well as more recently Atak and Kapetanios (2013) provide results of significant average frequencies of occurrence (jump times) and significant magnitudes of jumps (jump sizes). Barndorff-Nielsen and Shephard (2006) signify the importance of jumps in asset prices compared to continuous sample paths, whereas Todorov and Tauchen (2011) suggest that volatility is a pure jump process with jumps of infinite variation. In the present paper, the significance of jumps is measured via the mean magnitude of jumps $(\overline{J M})$ (Table 8), the ratio of the mean magnitude of the jump component of risk to the mean magnitude of the continuous component of risk $\overline{(\bar{R}}) 10$ (Table 9 ) and the average frequency of jump occurrence $(\bar{J}) 11$ (Table 10).

\subsubsection{Average magnitude of jumps $(\overline{J M})$}

The $R R$ realized range ( $M R$ monthly range) group of estimators has the highest (lowest) mean magnitude of jumps $(\overline{J M})$ series across all BRIC countries. The highest (lowest) $\overline{J M}$ series comes from the RRAYZ(MRAYZZ) estimator across BRIC Eurobonds, whereas the highest (lowest) $\overline{J M}$ comes from the Indian (Brazilian) Eurobond across the board of estimators.

In specific, regarding Brazil, the $R R$ realized range ( $M R$ monthly range) group of estimators has the highest (lowest) mean magnitude of jumps $\left(J M_{t}\right)$ series $(\bar{M})$ among groups, and the $R_{R}(G K(M R(Y Z))$ estimator among individual estimators. Regarding Russia and India, the $R R$ realized range ( $R V$ realized volatility) group of estimators has the highest (lowest) $\overline{J M}$ among groups, and the $R R_{A} Y Z \backslash(M R A Y Z)$ estimator among individual estimators. Regarding China, the $R V$ realized volatility ( $M R$ monthly range) group of estimators has the highest (lowest) $\overline{J M}$ among groups, and the RVAma adj) (MR(YZ) estimator among individual estimators.

For the group of realized volatility estimators, Indian (Brazilian) Eurobonds have the highest (lowest) mean magnitude of jumps $\left(J M_{t}\right)$ series $(\overline{M M})$ among BRIC countries. For the group of monthly ranges, Indian (Russian) Eurobonds have the highest (lowest) $\overline{J M}$ among BRIC countries. For the group of realized range-based volatility estimators, Indian (Brazilian) Eurobonds have the highest (lowest) $\overline{J M}$ among BRIC countries.

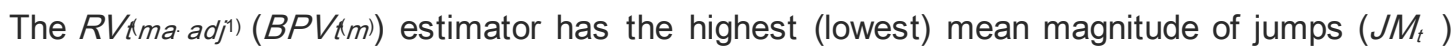
series $(\overline{J M})$ among the realized volatility estimators, across all BRIC Eurobonds. The MRtPar $\left(M R^{\prime}(Y Z)\right.$ estimator has the highest (lowest) $\overline{J M}$ among the monthly ranges, 
across all BRIC Eurobonds. The RRAYZ (RRARS) estimator has the highest (lowest) $\overline{J M}$ among the realized range-based volatility estimators, across all BRIC Eurobonds. Regarding the mean magnitude of jumps $\left(J M_{t}\right)$ series $(\overline{J M})$, all estimators are consistent and results change because of the informational content of BRIC bonds. All mean values of jump magnitude estimates for all bonds and estimators are t-test statistically significant. Moreover, the mean magnitude of jumps $\left(J M_{t}\right)$ series $(\overline{M M})$ coming from theoretical prices (theoreticalprice Jump magnitudes) is higher than the mean magnitude of jumps coming from actual market prices (actual-price Jump magnitudes). This result is evident in most of estimators and BRIC bonds. Across most of the eurobonds, the higher the expiry period, the higher the mean magnitude of jumps $\left(J M_{t}\right)$ series $(\overline{J M})$ is.

\subsubsection{Average magnitude of jump component of risk relative to the magnitude of the continuous component $(\overline{J R})$}

The $R V$ realized volatility ( $M R$ monthly range) group of estimators has the highest (lowest) mean $\left(J R_{t}\right)$ series $(\overline{J R})$ across all BRIC countries. The highest (lowest) $\overline{J R}$ comes from the $\left.\left.B P V_{(t m}\right)\left(M R^{(} R S\right)\right) \quad$ estimator across BRIC Eurobonds, whereas the highest (lowest) $\overline{J R}$ comes from the Chinese (Brazilian) Eurobond across the board of estimators.

In specific, regarding Brazil and Russia, the $R V$ realized volatility ( $M R$ monthly range) group of estimators has the highest (lowest) mean of $J R_{t}$ series $\overline{d R}$ ) among groups, and the $B P V(m)(M R(R S)$ ) estimator among individual estimators. Regarding India, the MR monthly range $(R V$ realized volatility) group of estimators has the highest (lowest) $\overline{J R}$ among groups, and the MRARS (RVima adj)) estimator among individual estimators. Regarding China, the $R V$ realized volatility (MR monthly range) group of estimators has the highest (lowest) $\overline{J R}$ among groups, and the $B P V(m)\left(M R{ }^{\prime} G K\right)$ estimator among individual estimators.

For the group of $R V$ realized volatility estimators and the group of $R R$ realized range estimators, Chinese (Brazilian) Eurobonds have the highest mean of $J R_{t}$ series $(\overline{J R})$ among BRIC countries. For the group of $M R$ monthly ranges, Indian (Russian) Eurobonds have the highest (lowest) $\overline{J R}$ among BRIC countries.

The $B P V(m)\left(R V t m a \cdot a d f^{2}\right)$ estimator has the highest mean of $J R_{t}$ series $\left.\overline{(R}\right)$ among the realized volatility estimators, across all BRIC Eurobonds. The MR(Par (MR $(R S)$ ) estimator has the highest (lowest) $\overline{J R}$ among the monthly ranges, across all BRIC Eurobonds. The $R_{R}\left(G_{K}\right)\left(R R_{(P a r}\right)$ estimator has the highest (lowest) $\overline{J R}$ among the realized range-based volatility estimators, across all BRIC Eurobonds.

Regarding the mean of $J R_{t}$ series $(\overline{J R})$, all estimators are consistent and results change because of the informational content of BRIC bonds. All mean ratios for all bonds and estimators are t-test statistically significant. Moreover, the mean of $J R_{t}$ series $(\overline{J R})$ coming from theoretical prices (theoretical-price Jump ratio) is higher than the mean Jump ratio coming from actual market prices (actual-price Jump ratio). This result is evident in most of estimators and BRIC bonds. Across most of the eurobonds, the shorter the expiry period, the higher the mean of $J R_{t}$ series $(\overline{J R})$ is. 


\subsubsection{Average frequency of jump occurrences $(\bar{J})$}

The $R V$ realized volatility (MR monthly range) group of estimators has the highest (lowest) average frequency of jump occurrence $(J t)$ series $(\bar{J})$ across all BRIC countries. The highest (lowest) $\bar{J}$ comes from the BPV(m) $\left(M R^{(} Y_{Z Z}\right)$ estimator across BRIC Eurobonds, whereas the highest (lowest) $\bar{J}$ comes from the Brazilian (Russian) Eurobond across the board of estimators.

For all $\mathrm{BRIC}$ countries, the $R V$ realized volatility (MR monthly range) group of estimators has the highest (lowest) average frequency of jump occurrence series $(\bar{J})$ among groups, and the $B P V(m)(M R(Y Z)$ estimator among individual estimators.

For the group of $R V$ realized volatility estimators and the group of $M R$ monthly ranges, Brazilian (Russian) Eurobonds have the highest average frequency of jump occurrence series $(\bar{J})$ among BRIC countries. For the group of $R R$ realized range-based volatility estimators, Russian (Chinese) Eurobonds have the highest $\bar{J}$ among BRIC countries.

The $B P V(m)(R V(m))$ estimator has the highest (lowest) average frequency of jump occurrence $(\bar{J})$ among the $R V$ realized volatility estimators, across all BRIC Eurobonds. The MRt Par $^{\prime}\left(M R^{\prime}(Y Z)\right.$ ) estimator has the highest (lowest) $\bar{J}$ among the MRmonthly ranges, across all BRIC Eurobonds. The $R R \wedge Y Z\left(R R^{(} R S S\right)$ estimator has the highest (lowest) $(\bar{J})$ among the $R R$ realized range-based volatility estimators, across all BRIC Eurobonds.

Regarding the mean frequency of jump occurrence $(J t)$ series $(\bar{J})$, all estimators are consistent and results change because of the informational content of BRIC bonds. All mean values of jump magnitude estimates for all bonds and estimators are t-test statistically significant. Moreover, the mean frequency of jump occurrence $(J t)$ series $(\bar{J})$ coming from theoretical prices (theoretical-price Jump frequency) is higher than the mean frequency of jumps coming from actual market prices (actual-price Jump frequencies). This result is evident in most of estimators and BRIC bonds. Across most of the eurobonds, the higher the expiry period, the higher the mean frequency of jump occurrence $(J t)$ series $(\bar{J})$ is.

Regarding the frequency of jump occurrence, Russian (Indian) Eurobonds have the highest (lowest) number of estimators for which the $\bar{J}$ is significant12. The $R V$ realized volatility (MR monthly range) group of estimators has the highest (lowest) number of Eurobonds for which the $\bar{J}$ is significant. The $\left.B P V_{t} m\right)\left(M R^{\star} Y Z Z\right)$ estimator has the highest (lowest) number of Eurobonds for which the $\bar{J}$ is significant.

\section{Conclusions}

Concluding remarks concern results across all significance-measures: two risk significancemeasures $(\bar{R}$, and $\overline{S R})$ and three jump significance-measures $(\overline{J M}, \overline{J R}$ and $\bar{J})$. The overall significance is evident when most of the significance measures are significant. The significance of each either risk- or jump-measure is indicated as reported in the empirical findings section. Firstly, findings are consistent as far as there are not many differences between the group of the two risk measures and the group of the three jump measures. 
Moreover, there are not many differences among the two risk measures and also among the three jump measures. Moreover, all risk and jump measures from theoretical prices are higher than those from actual prices, across bonds and estimators. Across most of the eurobonds and measures, the higher the expiry period, the higher is the significance of risk and jumps. This result is consistent with bond theory. The Chinese Eurobonds are the most significant, across the board of estimators. Among BRIC Eurobonds, the C15 the Eurobond is the most significant.

The $R V$ realized volatility group of estimators and the $M R$ monthly range group of estimators are the most significant (in terms of both risk and jumps) across all BRIC countries. The most significant estimators areBPV(m) bipower variation (high in risk and jumps) and MR $Y$ Y monthly Yang \& Zhang range (low in risk and jumps) across BRIC Eurobonds. All risk and jump significance-measures are consistent across the boards of estimators and BRIC Eurobonds.

For all BRIC countries, the $R V$ realized volatility ( $M R$ monthly range) group of estimators retrieves the highest (lowest) estimates of risk and jumps. The bipower variation (monthly Yang \& Zhang range) estimator produce the highest (lowest) estimates of risk and jumps.

For the $R V$ realized volatility group and the $R R$ realized range group of estimators, the Chinese (Brazilian) Eurobonds have the highest (lowest) estimates of risk and jumps among all BRIC Eurobonds (countries). For the group of MR monthly ranges, Brazilian (Russian) Eurobonds (Brazil) have the highest (lowest) estimates of risk and jumps among all BRIC Eurobonds (countries). Risk and jump estimates are higher (lower) for theoretical prices rather than actual prices for the $R V$ realized volatility ( $M R$ monthly range) group of estimators. The present paper suggests that theoretical prices are better to be used instead of the actual. This empirical implication may trigger research on incorporating the theoretical pricing of Eurobonds into modeling, forecasting and investing Eurobonds. As BRICs (Brazil, Russia, India and China) become much larger force in the world economy, the accurate measurement and the properties of BRIC Eurobonds risk will become more important in the international financial markets and academia. The direct implications concern pricing structured products, fund management, the predictability of risk, and international asset allocation.

\section{Acknowledgment}

The authors would like to thank the Editors Hamid Beladi and Carl R. Chen as well as two anonymous referees for their valuable suggestions that improved the manuscript.

\section{References}

1.

o Afonso et al., 2014

- A. Afonso, P. Gomes, A. Taamouti

o Sovereign credit ratings, market volatility, and financial gains 
o Computational Statistics and Data Analysis, 76 (2014), pp. 20-33

2.

o $\quad$ Ait-Sahalia et al., 2013

o Y. Ait-Sahalia, J. Fan, Y. Li

o The leverage effect puzzle: Disentangling sources of bias at high frequency

o Journal of Financial Economics, 109 (2013), pp. 224-249

3.

o Ait-Sahalia and Jacod, 2009

o Y.A. Ait-Sahalia, J. Jacod

o Testing for jump in a discretely observed process

o Annals of Statistics, 37 (2009), pp. 184-222

4.

o Ait-Sahalia and Jacod, 2011

o Y.A. Ait-Sahalia, J. Jacod

o Testing whether jump have finite or infinite activity

o Annals of Statistics, 39 (2011), pp. 1689-1719

5.

o $\quad$ Ait-Sahalia et al., 2012

o Y. Ait-Sahalia, J. Jacod, J. Li

o Testing for jumps in noisy high frequency data

o Journal of Econometrics, 168 (2012), pp. 207-222

6.

o Aloui et al., 2011

o R. Aloui, M.S.B. Aissa, D.K. Nguyen

o Global financial crisis, extreme interdependences, and contagion effects: The role of economic structure?

o Journal of Banking \& Finance, 35 (2011), pp. 130-141

7.

o An et al., 2014

o B. An, A. Ang, T.G. Bali, N. Cakici

o The joint cross section of stocks and options

o Journal of Finance, 69 (5) (2014), pp. 2279-2337

8.
o Andersen and Bollerslev, 1998
o T.G. Andersen, T. Bollerslev 
o Answering the skeptics: Yes, standard volatility models do provide accurate forecasts

o International Economic Review, 39 (1998), pp. 885-905

9.

- Andersen et al., 2001

o T.G. Andersen, T. Bollerslev, F.X. Diebold, P. Labys

o The distribution of exchange rate volatility

o Journal of the American Statistical Association, 96 (2001), pp. 42-55

10.

- Andersen et al., 2003

o T.G. Andersen, T. Bollerslev, F.X. Diebold, P. Labys

o Modeling and forecasting realized volatility

o Econometrica, 71 (2003), pp. 579-625

11.

o Andersen et al., 2010

o T.G. Andersen, T. Bollerslev, P. Frederiksen, M. Nielsen

o Continuous-time models, realized volatilities, and testable distributional implications for daily stock returns

o Journal of Applied Econometrics, 25 (2010), pp. 233-261

12.

- Atak and Kapetanios, 2013

- A. Atak, G. Kapetanios

- A factor approach to realized volatility forecasting in the presence of finite jumps and cross-sectional correlation in pricing errors

o Economics Letters, 120 (2013), pp. 224-228

13.

o Bandi, Russell and Yang, 2008

o F.M. Bandi, J.R. Russell, C. Yang

o Realized volatility forecasting and option pricing

o Journal of Econometrics, 147 (2008), pp. 34-46

14.

o Bandi, Russell and Zhu, 2008

o F.M. Bandi, J.R. Russell, Y. Zhu

o Using high-frequency data in dynamic portfolio choice

o Econometric Reviews, 27 (1-3) (2008), pp. 163-198

15. 


\section{o Bannouh et al., 2013}

o K. Bannouh, M. Martens, D. van Dijk

o Forecasting volatility with the realized range in the presence of noise and non-trading

o North American Journal of Economics and Finance, 26 (2013), pp. 535-551

16.

o Barndorff-Nielsen et al., 2011

o O. Barndorff-Nielsen, P. Hansen, A. Lunde, N. Shephard

o Subsampling realised kernels

o Journal of Econometrics, 160 (2011), pp. 204-219

17.

o Barndorff-Nielsen and Shephard, 2006

o O.E. Barndorff-Nielsen, N. Shephard

o Econometrics of testing for jumps in financial economics using bipower variation

o Journal of Financial Econometrics, 4 (2006), pp. 1-30

18.

o Bekiros, 2014

o S. Bekiros

o Contagion, decoupling and the spillover effects of the US financial crisis: Evidence from the BRIC markets

o International Review of Financial Analysis, 33 (2014), pp. 58-69

19.

o Bollerslev et al., 2013

o T. Bollerslev, D. Osterrieder, N. Sizova, G. Tauchen

o Risk and return: Long-run relations, fractional cointegration, and return predictability

o Journal of Financial Economics, 108 (2013), pp. 409-424

20.

o Busch et al., 2011

o T. Busch, B.J. Christensen, M. Nielsen

o The role of implied volatility in forecasting future realized volatility and jumps in foreign exchange, stock, and bond markets

o Journal of Econometrics, 160 (2011), pp. 48-57

1.

o Byun and Kim, 2013

o S.J. Byun, J.S. Kim

o The information content of risk-neutral skewness for volatility forecasting 
o Journal of Empirical Finance, 23 (2013), pp. 142-161

2.

o Cakir and Kadundi, 2013

o M.Y. Cakir, A. Kadundi

o Trade shocks from BRIC to South Africa: A global VAR analysis

o Economic Modelling, 32 (2013), pp. 190-202

3.

o Clements et al., 2008

o M.P. Clements, A.B. Galvao, J.H. Kim

o Quantile forecasts of daily exchange rate returns from forecasts of realized volatility

o Journal of Empirical Finance, 15 (4) (2008), pp. 729-750

4.

o Eraker et al., 2003

o B. Eraker, M. Johannes, N. Polson

o The impact of jumps in volatility and returns

o Journal of Finance, 58 (2003), pp. 1269-1300

5.

o Fang and You, 2014

o C.R. Fang, S.Y. You

o The impact of oil price shocks on the large emerging countries' stock prices: Evidence from China, India and Russia

o International Review of Economics and Finance, 29 (2014), pp. 330-338

6.

o Garman and Klass, 1980

o M.B. Garman, M.J. Klass

o On the estimation of security price volatilities from historical data

o Journal of Business, 53 (1980), pp. 67-78

o Full Text via CrossRef

7.

o $\quad$ Giot and Laurent, 2004

o P. Giot, S. Laurent

o Modelling daily Value-at-Risk using realized volatility and ARCH type models

o Journal of Empirical Finance, 11 (3) (2004), pp. 379-398

8.

o Goodliffe and Sberro, 2012 
- G. Goodliffe, S. Sberro

o The G20 after Los Cabos: Illusions of global economic governance

o International Spectator: Italian Journal of International Affairs, 47 (2012), pp. 1-16

9.

o Hansen et al., 2008

o P.R. Hansen, J. Large, A. Lunde

o Moving average-based estimators of integrated variance

o Econometric Reviews, 27 (2008), pp. 79-111

10.

o Henderson et al., 2006

o B.J. Henderson, N. Jegadeesh, M.S. Weisbach

o World markets for raising new capital

o Journal of Financial Economics, 82 (2006), pp. 63-101

11.

- Illueca and Lafuente, 2006

o M. Illueca, J.A. Lafuente

o New evidence on expiration-day effects using realized volatility: An intraday analysis for the Spanish stock exchange

o Journal of Futures Markets, 26 (2006), pp. 923-938

12.

o Jacquier and Okou, 2014

o E. Jacquier, C. Okou

o Disentangling continuous volatility from jumps in long-run-return relationships

o Journal of Financial Econometrics, 12 (3) (2014), pp. 544-583

13.

o Jiang and Tian, 2005

o G.J. Jiang, Y.S. Tian

o The model-free implied volatility and its information content

o Review of Financial Studies, 18 (2005), pp. 1305-1342

14.

o Julius, 2005

o D. Julius

o US economic power

o Harvard International Review, 26 (2005), pp. 14-18

15. 
o Lee et al., 2015

o B.S. Lee, M. Paek, Y. Ha, K. Ko

o The dynamics of market volatility, market return and equity fund flow: International evidence

o International Review of Economics and Finance, 35 (2015), pp. 214-227

16.

o Louzis et al., 2013

o D.P. Louzis, S. Xanthopoulos-Sisinis, A.P. Refenes

o The role of high-frequency intra-daily data, daily range and implied volatility in multiperiod Value-at-Risk forecasting

o Journal of Forecasting, 32 (2013), pp. 561-576

17.

o Martens and van Dijk, 2007

o M. Martens, D. van Dijk

o Measuring volatility with the realized range

o Journal of Econometrics, 138 (2007), pp. 181-207

18.

o McCulloch, 1971

o H.J. McCulloch

o Measuring the term structure of interest rates

o Journal of Business, 44 (1971), pp. 19-31

19.

o McCulloch, 1975

o H.J. McCulloch

o The tax adjusted yield curve

o Journal of Finance, 30 (1975), pp. 811-830

20.

o Parkinson, 1980

o M. Parkinson

o The extreme value method for estimating the variance of the rate of return

o Journal of Business, 53 (1980), pp. 6-15

1.

o Peristiani and Santos, 2010

o S. Peristiani, J. Santos 
o Has the US bond market lost its edge to the eurobond market?

o International Review of Finance, 10 (2010), pp. 149-183

2.

o $\quad$ Rogers and Satchell, 1991

o L. Rogers, S. Satchell

o Estimating variance from high, low and closing prices

o Annals of Applied Probability, 1 (1991), pp. 504-512

3.

o Seo and Kim, 2015

o S.W. Seo, J.S. Kim

o The information content of option-implied information for volatility forecasting with investor sentiment

o Journal of Banking and Finance, 50 (2015), pp. 106-120

4.

o Sevi, 2015

o B. Sevi

- Explaining the convenience yield in the WTI crude oil market using realized volatility and jumps

o Economic Modelling, 44 (2015), pp. 243-251

5.

o Steinbock, 2012

o D. Steinbock

o The Eurozone debt crisis: Prospects for Europe, China, and the United States

- American Foreign Policy Interests: The Journal of the National Committee on American Foreign Policy, 34 (2012), pp. 34-42

6.

o Tauchen and Zhou, 2010

o G. Tauchen, H. Zhou

o Realized jumps on financial markets and predicting credit spreads

o Journal of Econometrics, 160 (2010), pp. 102-118

7.

o Todorov and Tauchen, 2011

o V. Todorov, G. Tauchen

o Volatility jumps 
o Journal of Business and Economic Statistics, 29 (2011), pp. 356-371

8.

o Wang et al., 2008

o T. Wang, J. Wu, J. Yang

o Realized volatility and correlation in energy futures markets

o Journal of Futures Markets, 28 (2008), pp. 993-1011

9.

o Yang and Zhang, 2000

o D. Yang, Q. Zhang

o Drift-independent volatility estimation based on high, low, open and close prices

o Journal of Business, 73 (2000), pp. 477-491

10.

o Zhang, 2011

o L. Zhang

o Estimating covariation: Epps effect, microstructure noise

o Journal of Econometrics, 160 (2011), pp. 33-47

11.

o Zhang et al., 2005

0 L. Zhang, P.A. Mykland, Y. Ait-Sahalia

o A tale of two time scales: Determining integrated volatility with noisy high-frequency data

o Journal of the American Statistical Association, 100 (2005), pp. 1394-1411

12.

o Zhu and Lian, 2015

o S.-P. Zhu, G.-H. Lian

o Pricing forward-start variance swaps with stochastic volatility

o Applied Mathematics and Computation, 250 (2015), pp. 920-933

Corresponding author. Tel.: + 441522835634 .

1

Tel.: + 441522835612 .

$\underline{2}$

This acronym refers to the association of Brazil, Russia, India and China that first convened in June 2009 ostensibly in response to the fallout of the 2008-09 financial crisis. In reality, it was formed to offer an alternative framework of global governance anchored by the leading emerging economies (see, Goodliffe \& Sberro, 2012).

$\underline{3}$ 
Eurobonds are issued offshore, in a currency different from that of the market where the bond is arranged. The increased role of Eurobonds was signified by Henderson, Jegadeesh, and Weisbach (2006), among others.

$\underline{4}$

The recent financial crisis period had an international impact from February 2007 up to February 2010. This is the target period of the present study.

$\underline{5}$ Zhang, Mykland, and Ait-Sahalia (2005) showed that, in the presence of jumps, two-scales realized volatility (TSRV) estimates the integrated variance plus the sum of squared intraday jumps. A more recent analytical study is Zhang (2011).

$\underline{6}$

Jacquier and Okou (2014) is a recent study in jumps in monthly realized volatility series.

7

More recent studies on the empirical applications, the properties of this detection scheme, the properties of jumps series are Ait-Sahalia and Jacod (2011), Ait-Sahalia et al. (2012), and AitSahalia, Fan, and Li (2013).

8

These ratings were published on 12/31/2010. The Moody's ratings start from $B B$ - (the worst rating) to the $\mathrm{A}+$ (best rating) in the following order: $\mathrm{BB}-, \mathrm{BB}, \mathrm{BB}+, \mathrm{BBB}-, \mathrm{BBB}, \mathrm{BBB}+, \mathrm{A}-$, A, A +.

9

The asymptotic properties for their jump detection scheme were provided by BarndorffNielsen and Shephard (2006) and Andersen et al. (2010) as well.

$\underline{10}$

Significance is indicated if the mean magnitude of the jump component of risk relative to the magnitude of the continuous component $(\overline{J R})$ is higher than 1 .

$\underline{11}$

Significance is indicated if the average frequency of occurrence of jumps $(\bar{J})$ is higher than $50 \%$.

$\underline{12}$

Significance is indicated if the average frequency of jump occurrences $(\bar{J})$ is higher than $50 \%$. Copyright $\odot 2015$ Published by Elsevier Inc.

Note to users: Corrected proofs are Articles in Press that contain the authors' corrections. Final citation details, e.g., volume and/or issue number, publication year and page numbers, still need to be added and the text might change before final publication.

Although corrected proofs do not have all bibliographic details available yet, they can already be cited using the year of online publication and the DOI, as follows: author(s), article title, Publication (year), DOI. Please consult the journal's reference style for the exact appearance of these elements, abbreviation of journal names and use of punctuation. 
When the final article is assigned to an volumes/issues of the Publication, the Article in Press version will be removed and the final version will appear in the associated published volumes/issues of the Publication. The date the article was first made available online will be carried over. 